LBL- I 1830

DE83 004054

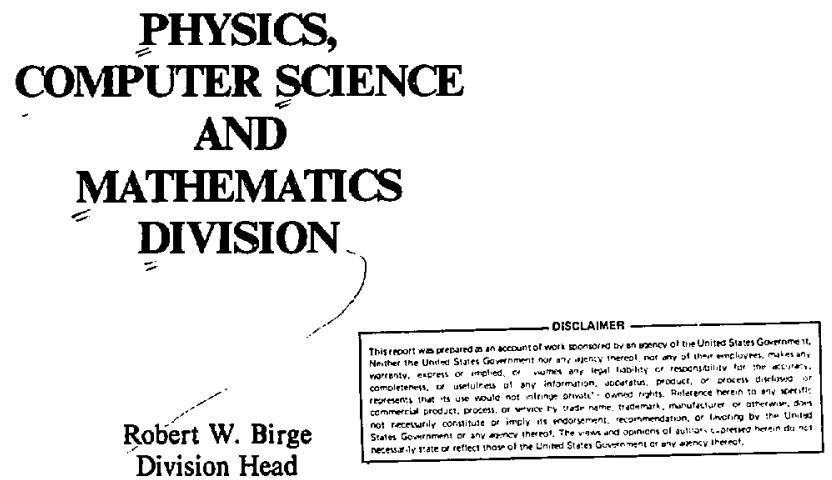

\title{
ANNUAL REPORT,
}

\section{January - 31 December 1981}

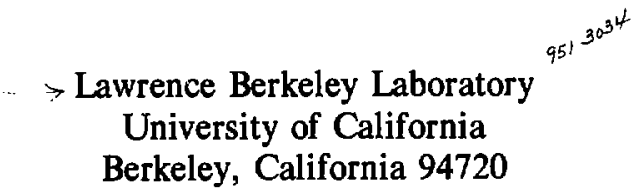

Major portionis of this work were supported by the Office of Energy Research, Divisions of High Energy and Nuclear Physics, Basic Energy Sciences, and Health and Environmental Research, and the Energy Information Administration, Statistical Support Division of the U.S. Department of Energy under Contract No. DE-AC03-76SF00098, Additional support was provided by the U.S. Department of Labor, the U.S. Department of Defense, the National Science Fcundation, the National Aeronautics and Space Administration, the National Oceanographic and Atmospheric Administration, the U.S. Army Corps of Engineers, and the University of California. 


\section{EXPERIMENTAL PHYSICS}

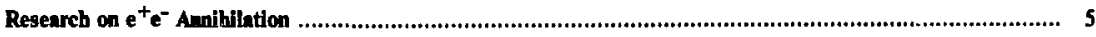

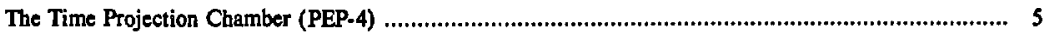

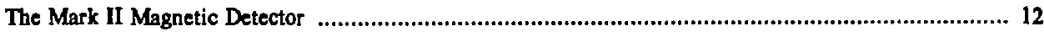

Free Quark Search ....................................................................................................... 17

PEP Experimental Facilities Coordination .......................................................................... 18

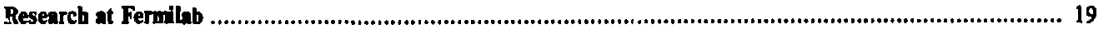

Hadron Calorimeter for CDF Collaboration ............................................................................ 19

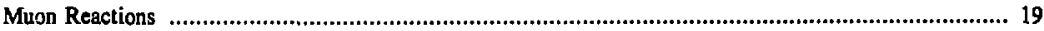

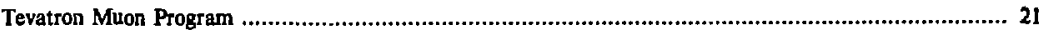

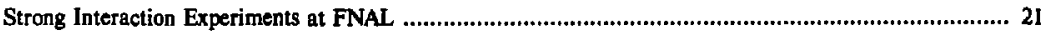

Experiments Using Polarized Proton and Antiproton Beams ....................................................... 21

Search for Effects of a Rlght-Handed Gauge Boson ......................................................................... 22

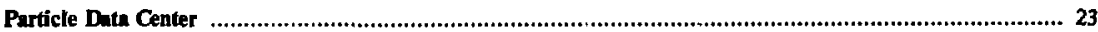

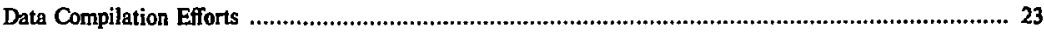

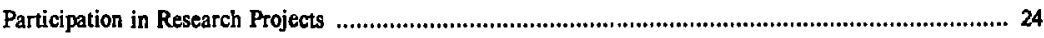

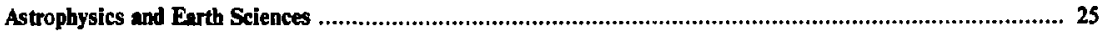

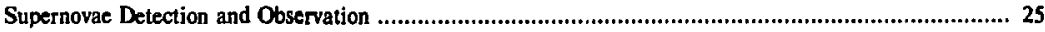

Cosmic Background Radiation/Primordial Blackbody Radiation .............................................. 25

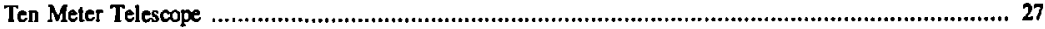

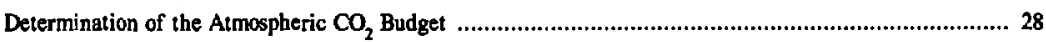

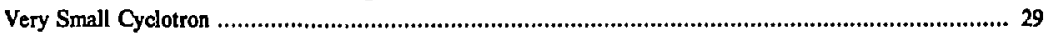

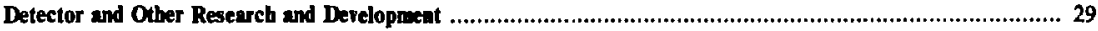

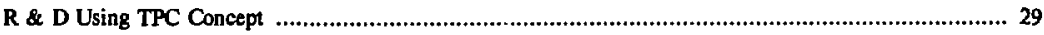

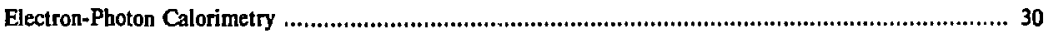

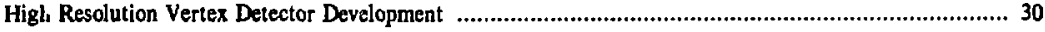

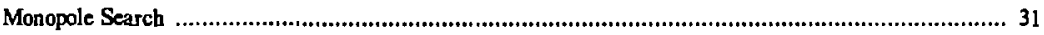

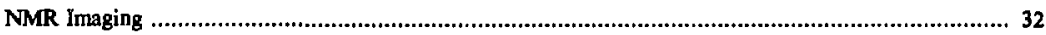

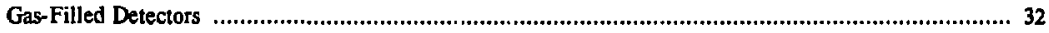

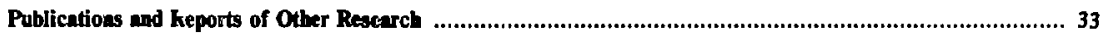

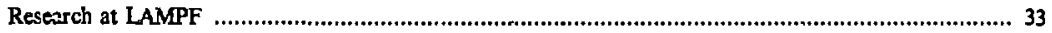

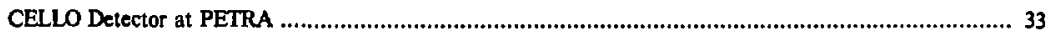

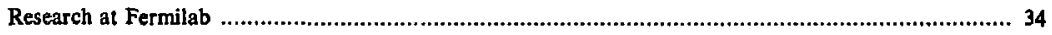

Kaonic-Atom X Rays ............................................................................................................. 34

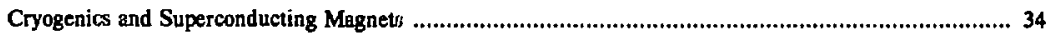

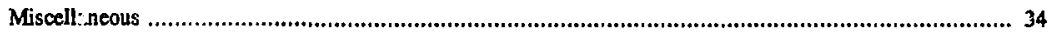

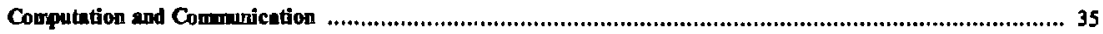

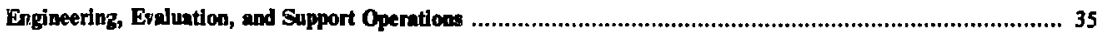




\section{THEORETICAL PHYSICS RESEARCH}

Particle Physics

Henvy Ion Fusion

\section{COMPUTER SCIENCE AND MATHEMATICS}

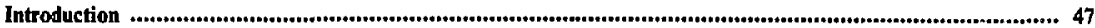

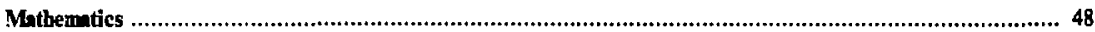

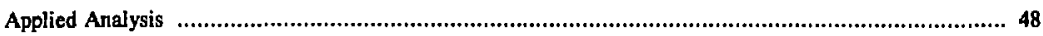

Computational Mathematics .......................................................................................... 48

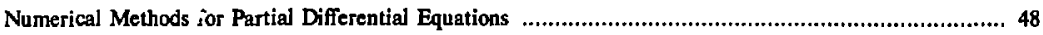

Mathematical Software and Consulting Services ..................................................................... 50

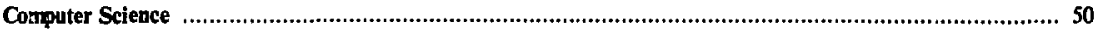

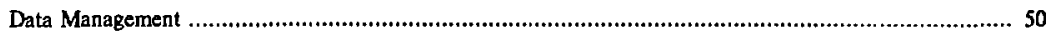

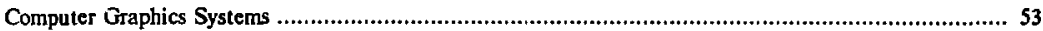

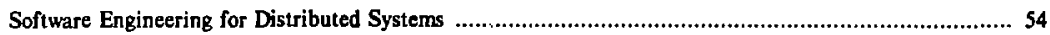

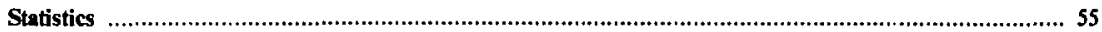

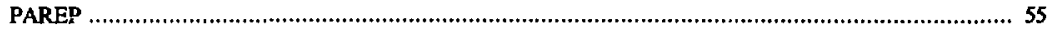

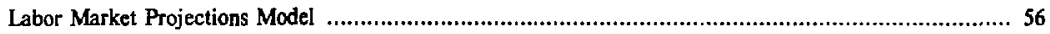

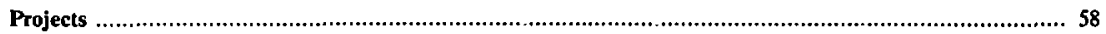

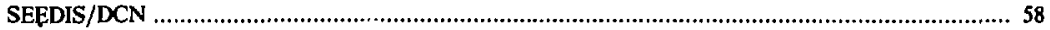

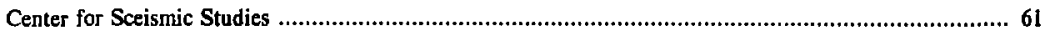

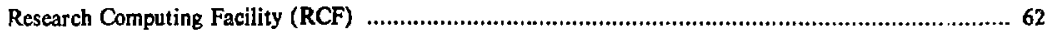

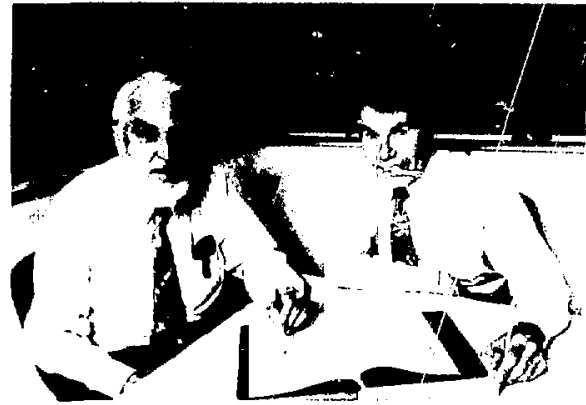

XBB 82127246

R. W. Birge (left) educates J. D. Jackson in planning and budgetary matters. Like neutrinos, division heads apparently corne with only one helicity. 


\section{INTRODUCTION}

This report summarizes the research performed in the Physics, Computer Science and Mathematics Division of the Lawrence Berkeley Laboratory during calendar year 1981. The major activity of the Division is research in high-energy physics, both experimental and theoretical, and research and development in associated technologies. A smaller, but still significant, program is in computer science and applied mathematics. At the beginning of 1981 there were roughly 200 people in the Division active in high-energy physics, not all full-time; at the end of 1981 the number was approximately 150 . In computer science and mathematics, the staff remained stable at about 46.

The Division draws considerable strength from a close association with the Berkeley campus of the University of California. Or the total Department of Physies faculty of 60, 25 have their research activities within the Physics CSM Division of LBL, 17 in experiment and 8 in theory. Also from the Berkeley campus are about 45 Physics graduate students, working at the Laboratory for their Ph.D. thesis research in particle physics. At the Laboratory itself, in high-energy physics there are 20 Staff Serioz Scientists, roughly equivalent to tenured faculty on campus, 15 Stafi Scientists with indefinite appointments, and 16 postdoctoral or equivalent term appointees. The technical and clerical/administrative staff in high-energy physies now number 20 and 13, respectively.

In the Department of Computer Science and Mathematics there are 30 permanent Staff Scientists, 8 term appointees, and 1 Staff Senior Scientist, plus one Faculty Senior Scientist and 6 Associated Faculty, and a small number of graduate students. The relative size of Computer Science to Mathematics is approximately 3:1.

In addition to the subject areas of the Division's title, research is pursued in astrophysics, geoscience, and pure astronomy.

During the year under review the Division devoted roughly half its effort to the final construction stages of the Time Projection Chamber and other equipment for the PEP-4 facility at SLAC. The year was marked by the successful passage of milestone after milestone-the two-sector test of the TPC with cosmic rays in July 1981, the full TPC test in November 1981, and the roll-in onto the PEP beam line on 6 January 1982. For the LBL participants and their collaborators from other institutions, 1981 was a year of great achievement on the PEP-4 experiment.

In other $\mathrm{e}^{+} \mathrm{e}^{-}$experiments, the Mark II detector continued its productive data-taking at PEP. Among the discoveries during 1981 were a first measurement of the lifetime of the tau lepton and firm establishment of scaling violations with energy for the inclusive pion cross section. The Free Quark Search conducted its experimental run during the first half of 1981 and completed data analysis by the end of the year. No free quarks were found and limits on the production cross section in $\mathrm{e}^{+} \mathrm{e}^{-}$collisions were set.

In other areas, the final stages of data analysis, particularly for the structure functions, proceeded for the inelastic muon scattering experiment performed at Fermilab, a muon polarimeter experiment was developed and mounted at TRIUMF to probe for the presence of right-handed currents in muon decay, and the design and then construction began of fine-grained hadron calorimeters for the end caps of the Colliding Detecter Facility at Fermilab. The Particle Data Group intensified its activities, despite financial constraints, as it proceeded toward production of a new edition of its authoritative Review of Particle Properties carly in 1982.

- To maintain an outstanding research program in a rapidly changing field like high-energy physics, it is necessary continually to explore new experimental techniques and devices. Within the Division, detector research and development is motivated by the desire to improve the evolving existing facilities and the desire to strike out in new directions. Because the TPC was invented here, it is natural that a considerable fraction of the $R \& D$ on detectors should center around the TPC concept-high density TPC's, either liquid or very high pressure gas, as well as improvements for the existing TPC of PEP-4. Among other R \& D projects during 1981 were a ring-imaging Cerenkov detector combined with a TPC, electron-photon calorimetry, very high-resolution detectors, and gas-filled multielectrode detectors.

During 1981 the Theoretical Physics Group pursued a diverse spectrum of research in its own right and also interacted effectively with the experimental program.

The Division's modest activities in astrophysics include continuing study cf the anisotropy of the cosmic background radiation, its polarization and spectrum, and initiation of an automated supernova search. Research and development continued on the segmented mirror for the ten-meter telescope proposed by the University of California.

Activities in the Compliter Science and Mathematics Department encompassed networking, database management, software engineering, and computer graphics, as well as basic research in nonlinear phenomena in combustion and fluid flow. 
For the Division, the year 1981 may well stand as a watershed. It was a year of great achievement but also, at the end, a year of crisis brought on by financial pressures and uncertainty about the future. For considerable earlier time a gradual transformation had been taking place, driven by the enormity of the TPC project. The traditional autonomous group structure had been breaking down, to be replaced by a program built around projects. Vestiges, and sometimes more, of the groups remain, and a totally smooth administrative replacement has yet to emerge, but the old regime is gone. A new mode of operation is emerging; with centralized planning and enhanced capability, we hope to seize and exploit successfully appropriate physics opportunities. The Division has unique capabilities to offer the national high-energy physies program because of its superb staff, its situation inside a laboratory with extensive and first-class technical support, and its close coupling to a great university campus. These qualities must surely emerge as you read the following pages.

Presiding over the Division for the past eight-and-one-half years (1 July, 1973 - 31 December 1981) as Head and Associate Director of the Laboratory has been Robert W. Birge. He has guided the research program most effectively during a glorious period for high-energy physics. In his first year and a half as Head, the revolutionary discovery of the psi particles was made by a joint LBL-SLAC team and (for the first one) by an MIT-BNL group. For the next five years, the Mark I and then the Mark Il delector group held center stage as exciting discoveries followed in rapid succession. Midway in his term of office the TPC was invented and parlayed daringly into the impressive device now at the heart of the PEP-4 facility. These are but a few of the outstanding achievements of tbe LBL scientists working under Birge's eye. His years as Head of the Division will be remembered fondly for his calm demeanor, his balanced scientific judgment, his sympathy and understanding, and his utter fairness and integrity. For his part he can take pleasure in a job well done in sometimes trying circumstances. For the Division and the Laboratory I say thank you, Bob Birge.

J. D. Jackson

Head 


\section{EXPERIMENTAL PHYSICS}


Abrams, Gerald S.

Armstrong, Frances E. Ashicy, Robert P.

Balke, Brisn

Barrera, J ssephine R. Bingham, Harry $H$.

Birge, Roivert W.

Bistirlich, lames

Blackman, , Jonald L

Blondel, Alain

Briges, Donald D.

Brink, Aslette

Bross, Alan D.

Buckingram, Sharon

Bucksbaum, Dessa

Buffington. Andrew

Burge, James $\mathbf{W}$,

Carithers, William C.

Caron, John L.

Chamberlain, Owen

Chen, Chaoqing

Chew, Denyse M.

Chinowsky, William

Clark, Alan R.

Covey. Charles L

Crawford, Frani.S.

Crawford, Theda W.

Crebbin, Linda J.

Crowe. Kenneth $\mathbf{M}$.

Culver, John A.

Dahl, Orin I.

Daly, Tim

Davenport, Julia A

Day, Christopher T.

Del Guerra, Alberto

Delpierre, Pierre

Deneau. Meivin G.

Dodge. William J.
Eberhard, Philippe H.

Elola, Mark A

Ejy, Robert E.

Fancher, David 1.

Frank-Dieterle, Christina A

Fujii, Hirofumi

Fuzesy, Raymond Z

Gabioud, Bernard

Galtieri, Angela B.

Galtieri, Paolo E.

Garnjost, Margaret A

Gibson, George $\mathrm{H}$.

Gidal, George

Goldhaber, Gerson

Goozen, Frederick R

Graham, Christina K.

Gu, Weixin

Harding. Peter C.

Heuptman, John M.

Heck, Burkhard W.

Horne, Charles P.

Hunkler, Angela

Iwasaki, Hiroyuki

Johansen, Walter $\mathbf{E}$.

Jancs, Dorne $\mathbf{G}$.

Kadyk, John A

Kamae. Tunyos hi

Kaplan, Selig N.

Kastning, Vivian E

Kelly. Robert L.

Kennedy, Jane

Kenney, Robert L.

Kerth, Letoy $T$.

Koellner, Werner $\mathbf{O}$.

Kolier, Richard R.

Koop, Beverly C.

Kramer, Bridget R.
Lankford, Andrew J.

Lee, Edward $E$.

Loken, Stewart C.

London, Georges W.

Long, Michael R.

Ludwig, Laura A.

Lynch, Gerald R.

Lys, Jeremy E.

Madaras, Ronald J.

Martin, Philip S.

Martin, Teri E

Marx, Jay $\mathbf{N}$.

Mast, Terry S.

McCann, Earl

Miller, Jeanne $\mathrm{M}$.

Mulere, Terrance A.

Muller, Richard A.

Murphy, Patricia A

Nelson, Jerry E.

Nemethy, Peter

Nuzum, Timothy $E$

Nygren, David R.

Oddone, Piermaria J.

Pardoe, Barric

Pennypacker, Carlton

Perez-Mendez, Viclor

Peterson, Steven

Pripstein, Morris

Przbylski, Gerald T.

Pun. Ting P.

Reinsch, Paul S.

Rice, Harriett J.

Reynolds, Wendy L

Rittenberg, Alan

Robrish, Peter R.

Ronan, Michael T.

Ross, Ronald R.
Sagle, Allan L. Scales, Laura C.

Schnurmacher, Gerald L.

Shapira, Gilbert

Sherman, Steven

Shigley, Douglas B.

Shimada, Tohuzo

Shinsky, Kirk A

Smith, Garth W.

Smits, Robert G.

Smool, Goorge F.

Soroka, David P.

Steiner, Herbett $M$.

Steverson, M. Lynn

Strait, James B.

Strovink, Mark W.

Tans, Petrus P.

Taylor, John D.

Trilling, Csorge

Tripp, Robert D.

Trippe, Thomas G.

Van Slyke, Harold W.

Vercelli, Donna L.

Vuletich, Tony

Wang, Yunyong

Waters, John N.

Weber, Paul W.

Weber. Thomas F.

Wenzel, Willam A

Whipple, Edgar $T$.

Wiedenbeck, Pamela E. Williams, Gary A

Wissmar, Jocl C.

Wohl, Charles $\mathbf{G}$.

Yamamoto, Hiroaki

Yost, George

Zhao, Guozhe

\section{GRADUATE STUDENT RESEARCH ASSISTANTS}

Aihara, Hiroaki

Amidei, Dante E.

Derby, Kevin A.

Dieterle, Willam E.

Dillon, John B.

Falon, Mark W.

Gary, John W.

Gold, Michael

Goulding, Laurence J.
Hadley, Nicholas J.

Huth, John E,

Jodidio, Alexander E.

Krivicich, Joseph

Mannucci, Anthony

Markewicz, Thomas W.

Meyers, Peter D

Mireshghi, Ali

Moses, William
Nelson, Mark

Patrick, James

Rouse, Forest

Rowson, Peter

Schellman, Heidi $M$.

Schnetzer, Stephen

Shapito, Marjorie

Sheldon, Paul

Sleatord, Bradley
Stoker, Devid

Toge, Nobulazu

Van Tyen, Raymond

Vella, Eric N.

Weinstein, Paul

Welch, James J.

Yamauchi, Mesanori

Zarbalthsh, Farshid 


\title{
EXPERIMENTAL PHYSICS
}

\author{
Research on $\mathrm{e}^{+} \mathrm{e}^{-}$Annihilation
}

Experiments at the PEP electron-positron storage ring at the Stanford Linear Accelerator Center continued during 1981 to occupy a dominant place in the research program of the Physies Division. Major involvement occurred in three different areas. The largest single effort was on the PEP-4 experiment, mounted by a collaboration of physicists from LBL, UCLA, UCR, Johns Hopkins, Yale and Tokyo. The second major involvement was in the Mark II detector, running successfully at PEP after many triumphs at SPEAR. This work continued a long and fruitful collaboration with physicists from SLAC and Harvard. The third and more modest effort was the Free Quark Search, in collaboration with Stanford, SLAC, and Hawaii. In addition, LBL staff members continued to coordinate the experimental facilities at PEP.

\section{The Time Projection Chamber (PEP-4)}

During 1981 great progress was made on the detector based on the Time Prujection Chamber (TPC). During this time the fabrication and testing of most of the major components were finished, the various subsystems were integrated and tested as a complete detector, and the whole PEP-4 detector was rolled into the PEP beamline in Interaction Region 2 (IR2) at SLAC.

\section{TPC Chamber}

All twelve TPC proportional chamber endplane sectors were carefully fabricated and instrumented. Each of the sectors was tested at full operating voltage and pressure, and all performed very satisfactorily. Maps of the sense wire gain over the whole area of each sector were made using an external radioactive source. It was found that the gain was quite stable and reproducible (to approximately $1 \%$ ) and that it was quite uniform among the sense wires (to approximately $2 \%$ ).

The TPC high voltage syster, which creates the uniform stable electric field inside the TPC, had its final tests at full design voltage and pressure. This system includes the high voltage insulation, field cages, midplane electrode, cable and power supply. The high voltage system operated stably at $100 \mathrm{kV}$ for several weeks, with no evidence of anly corona being seen. The ratios of the field cage resistor chain currents were constant to several parts in 100,000 , and preamplifiers connected to antenna wires inside the high-voltage structure were very quiet and showed no evidence of any discharges. One of the final TPC sectors was installed for part of the tests, and its preamplifiers were also very quiet and stable. After the tests were completed the high-voltage system returned to the shops for a new, flatter midplane electrode and a final precise mechanical alignment. It was then delivered to IR2 at PEP at the end of May. (See Figures 1,2,3,4.)
The full high-voltage system and two endplane sectors were integrated at IR2 for the first cosmic ray test of the final TPC detector at full operating voltage $(75 \mathrm{kV})$ and pressure $(8.5 \mathrm{~atm})$. Cosmic ray tracks drifting the full $1-\mathrm{m}$ length of the TPC were immediately seen at the beginning of the test, on July 1, 1981 . The TPC operated well, with spatial and $\mathrm{dE} / \mathrm{dx}$ resolutions close to those expected. Gas purity was good, and electron capture over the $1-\mathrm{m}$ drift distance was small $(<10 \%)$. It was found, however, that the tracks had spatial distortions of several centimeters near the inner and outer field cage surfaces. Tests were maje to find the cause of the spatial distortions, and it is now believed that they are electrostatic in origin, possibly due to unexpected potentials on the field cage surface between the field-shaping electrodes.

After the TPC cosmic ray test, the field-shaping copper strips on the high voltage field cages were modifiod by making them wider. This reduced the spatial distortions of the tracks near the field cage surfaces $b_{j}$ a factor of ten, to several millimeters. All twelve sectors were then added to the high voitage system, and the full TPC was installed inside the conventional magnet at the end of September. After installing the plumbing for the source actuators and sector cooling, cabling all the sectors to the 10-atm feedthrough ring, and checking out and calibrating the sectors and electronics, the IPC was ready in early November for the full PEP-4 system coemic ray teats. (See Figure 5.)

\section{TPC Gas System}

The TPC gas system, consisting of tube trailer supply sources, a gas cart facility for mixing, purifying and swi: ;hing gases, accumulator storage tanks, interconnecting piping, vacuum pumps and interlocks was completed and became fully operational. It supplied $80 \%$ argon - $20 \%$ methane gas to the TPC during the summer TPC cosmic ray test and the November PEP-4 


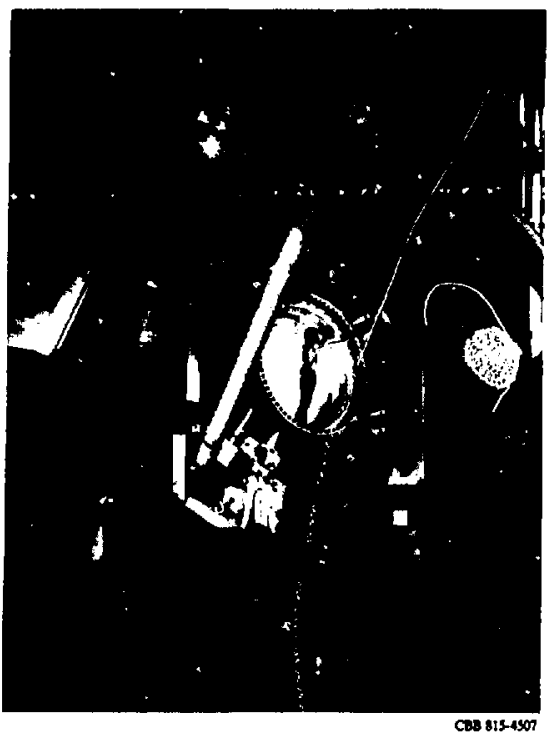

Fig. 1. Pressure tank of the PEP-4 experiment waiting for the TPC to be inserted at Interaction Region 2 (IR-2) of the large electronpositron storage ring called PEP at the Stanford Linear Accelerator Center. David Badtke of Johns Hopkins, a member of the collaboration, sets the scale.

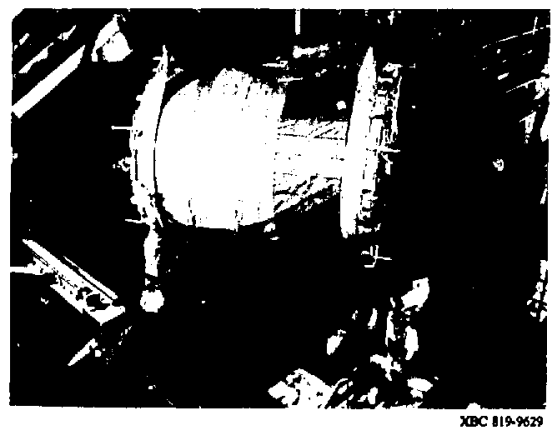

Fig, 2. Time Projection Chamber arriving at IR-2. The 2-m long by $2-\mathrm{m}$ diameter detector is being guided by Harold Van Slyke (head not visible) and Wayne Lee.

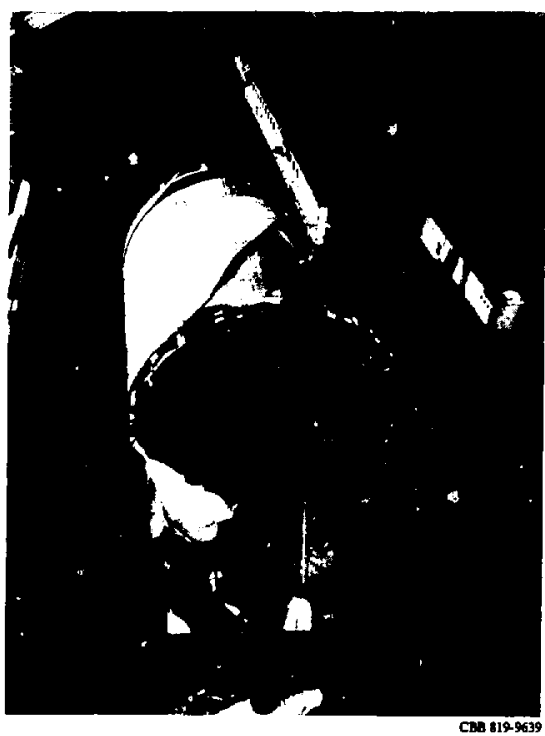

Fig. 3. TPC being jockeyed into position by Fred Catania (SLAC) on the left and Wayne Lee on the right.

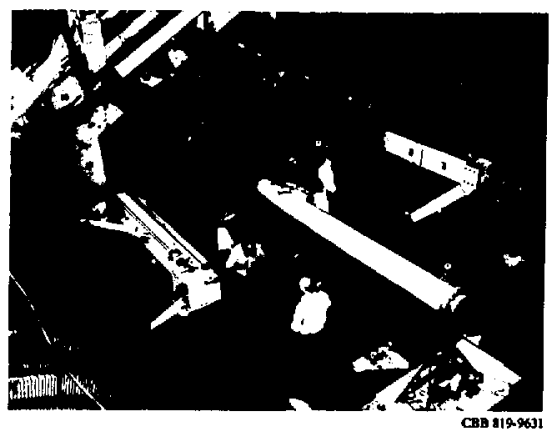

Fig. 4. TPC now in place inside the pressure vessel. A kneeling Harold Van Slyke and Roy Kerth discuss the next step, while Dave Fancher, Ron Madaras and others admire the fit. 


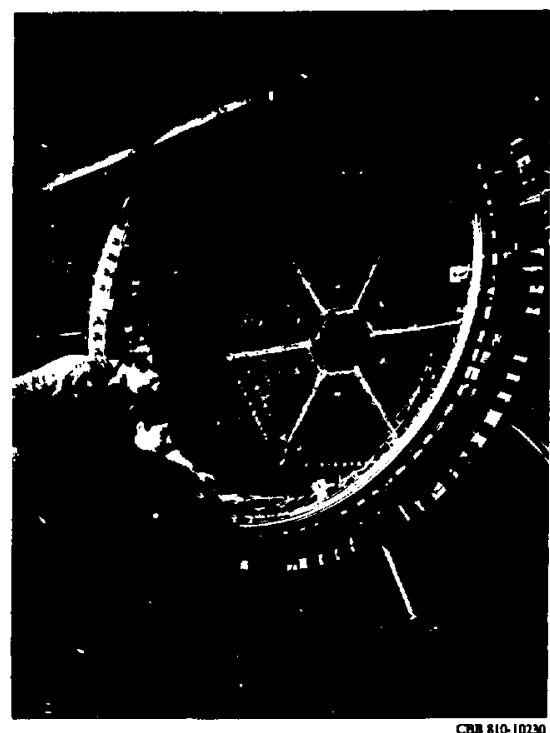

Fig. 5. End face of TPC shows elaborate pattern of electric cabling required to get the timing and ionization information from the TPC end planes to the electronic house. Marjorie Shapiro and Garth Smith make final adjustments.

system cosmic ray test. The gas system was able to supply gas with an oxygen contamination as low as 0.2 ppm. For the PEP-4 system cosmic ray test, however, the contamination increased by a factor of ten by the time the gas entered the TPC. After several studies, it is now believed that the problem is due to inadequate gaskets at the joints of the exterior manifold that distributes the gas to the TPC. This will be corrected in 1982.

\section{Electronics}

The electronics system is essentially complete. Almost all shaper, amplifier, $C C D$, digitizer and readout master controljer boards have been produced, installed and tested. The Large Data Buffer (LDB) was completed and moved to IR2 in April and has been tested and brought online. All 37 read-out lists have been debugged and are up and running. The test pulsc system for the TiC will require additional work in 1982 to make it comple:ely satisfactory.

The fabrication of the charged particle trigger electronics has been completed, and the system has been thoroughly debugged. The TPC ripple trigger, majority trigger, and midplane source trigger were studied during the two TPC cosmic ray runs. In addition, a special cosmic ray trigger was developed for those runs. The trigger control and interrupt system was completed and used in tests of the trigger system and the cosmic ray runs. The Inner Drift Chamber and associated trigger electronics were used with the UC Riverside group in a beam run at PEP to check the trigger operation and to study backgrounds. The neutral particle trigger for the Pole Tip Calorimeter was designed and partially fabricated. It will be completed early in 1982 .

The hardware and software for the monitor system are essentially complete, except for the superconducting magnet monitoring, which has been postponed until 1982. The system is monitoring approximately 900 different channels of voltages, currents or thermistor resistances. It checks the readings against allowed limits and gives an alarm if the reading is outside the limits.

An interlock system to protect personnel and equipment has been designed, fabricated and implemented for the TPC fieid cage high voltage system and the TPC gas system. The state of the interlock system is annunciated in the PEP-4 Control Room, as well as the Gas House and Electronics House.

\section{PEP-4 Computer System}

The TPC VAX 1!/780 computer system, used for online data taking, was moved to IR2 in April. Two megabytes of memo-y were added to it, and all of its hardware is now complete. It has two 6250-bpi tape drives, and four megabytes of memory.

The PDP 11/70 computer system, used for online data taking and hardware testing and calibration, is complete. It has one 6250-bpi tipe drive, and 1.5 megabytes of memory.

The PEP 11/04 computer used with the hardware monitoring system, is complete.

The physics division VAX 11/780 was installed in the LBL Computer Center in January. It is used by PEP-4 for offline data analysis. It has two 6250-bpi tapdrives, and four megabytes of memory. Another tape drive will be installed in 1982 .

DECNET links together all of the above computers, as well as providing links to the VAXs of the PEP-4 collaborating institutions, the VAXs of other PEP experiments, and the other two VAXs of the LBL Computer Center.

\section{Soltware}

During 1981 the PEP-4 software had its first use in the acquisition and analysis of cosmic ray data from the TPC and other detectors in PEP-4. Right at the beginning of the TPC cosmic ray test the software was 
able to successfuily read the data in, analyze the data and find tracks, display cosmic ray events on a CRT, and write the data to tape. Many processes are now included in the $\mathrm{VAX}$ realtime program:

MASTER to initiate and control the running of the other processes listed below

EVENTSIN to read in the data

TAPEOUT to write the data to tape

ANL to analyze the data

TALLY to make histograms

OED to display events on a CRT

ALARM to handle alarm messages

COM to handle communication between the user and the other processes.

A continual effort has gone on to improve the operation of the VAX programs using the cosmic ray data, and especially to increase the efficiency and speed of the real-time system. In addition, data from the monitor system and electronic calibration, which are essential for achieving precise spatial and $\mathrm{dE} / \mathrm{dx}$ resolutions, have been integrated into the analysis program on the VAX.

On the PDP 11/70 the supervisor programs that handle intersupts and communication with the trigger electronics, that coordinat; the ieading of the data from the LDB, and that coordinate the test pulsing of the electronics and the reading and writing of registers in the electronics are all completed and well tested. Programs have been implemented for the calibration of the electronics, data quality monitoring, initializing the clectronics, performing diagnostics on the electronics, and accumulating and displaying histograms.

\section{Conventional Magnet Coil}

A conventional magnet coil capable of pr juiding a 4-kG magnetic field was fabricated and tested during 1981. The coil was wound in June. The high voltage and water connectors were then added, the inner and outer heat silields were installed, the Outer Drift Chamber was mounted around the coil, and the coil was insiatled in the magnet iron in IR2 in September.

\section{PEP-4 System Cosmic Ray Test}

During November the full TPC, Inrs and Outer Drift C'M mbers, Muon Chambers and Pole Tip Calorimeters (but excluding the Hexagonal Calorimeter) were assembled together in their final configuration and tested for one month using cosmic rays. The test used the full PEP-4 data acquisition system, trigger system, and on-line and off-line software programs. Data were taken for all the detectors, showing that they were all performing satisfactorily.
Data for the TPC were taken under a variety of operating conditions: pressure at 1.0,4.0 and $8.6 \mathrm{~atm}$ of $80 \%$ argon - $20 \%$ methane gas; TPC electric field from 15 to $100 \mathrm{kV} / \mathrm{m}$; and magnetic field of $0,+3.9$ and $-3.9 \mathrm{kG}$. The TPC performed very well. By comparing the energy loss of costnic rays in two opposing endplane sectors, it was possible to determine that the $\mathrm{dE} / \mathrm{dx}$ resolution is unsurpassed by any other large detector in high energy physics today. (See Figures 6 and 7.) An analysis of the power of the track reconstruction is shown in Figures $\mathbf{8}$ and 9, where an electron spirals down the length of the chamber. Under the same conditions, the TPC spatial resolution in the plane orthogonal to the axial drift direction was measured,

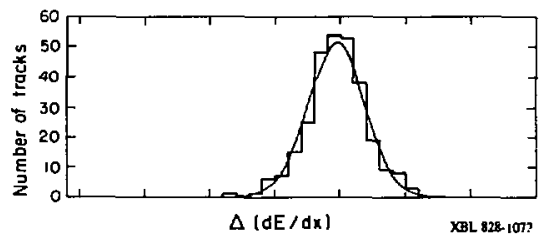

Fig. 6. Measured distribution of differences of ionization of cosmic ray muons in two different sectors of the TPC. The width of the Gaussian fit corresponds to a resolution of $3 \%$ in $\mathrm{dE} / \mathrm{d} \mathbf{x}$.

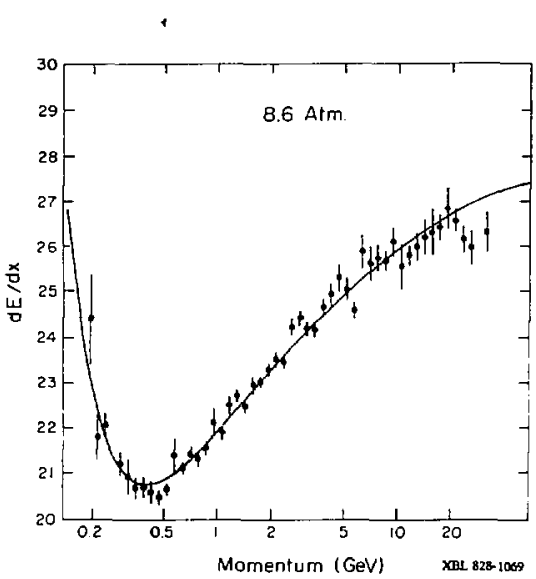

Fig. 7. Measured relative values of $\mathrm{dE} / \mathrm{dx}$ versus muon momentum in the TPC, showing the relativistic rise. Note the suppressed zero for the ordinate. The curve is theoretical, with its magnitude adjusted for a best fit. 


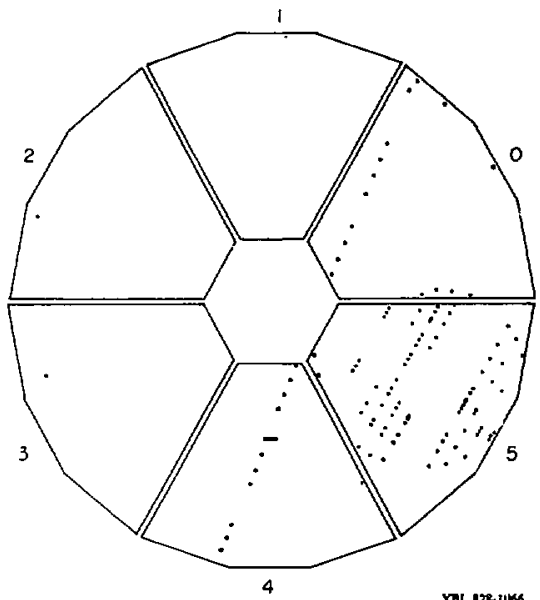

Fig. 8. Computer display of the preliminary endview information on "hits" of the pad rows of the TPC end-plane for an election spiraling in the chamber.

\section{All sectors $r$ vs $Z$}

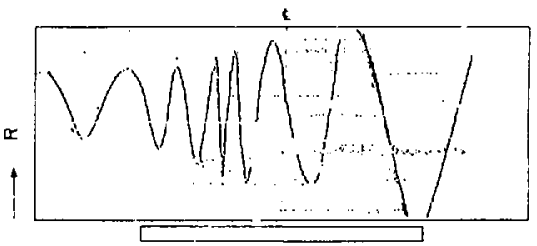

Z

XAL 828-3013

Fig. 9. Computer display of the longitudinal (tempora) information on the sector wires. The horizontal direction is parallel to the axis of the TPC. The vertical direction is radial, will all six sectors folded together. The computer makes a preliminary fit to the track of a spiraling election.

with a preliminary result of $250 \mu \mathrm{m}$. Both of these results are expected to improve with further calibration of the delector and the electronics, and with more analysis of the data.
Because the TPC and other detectors operated so successfully in the cosmic ray test, the decision was made to move PFP-4 into the PEP beam, beginning in December.

\section{Hexagonal Calorimeter}

All of the production tooling for the fabrication of the hexagonal calorimeter modules, such as the wire binding machine and the laminate grooving machine, have been completed and made operational. All of the jigs and fixturing for grooving, drilling, cutting, suldering, gluing and stacking the laminate have been prepared. By the end of 1981, two of the six hexagonal calorimeter modules were fabricated, tested and installed in the FEP-4 detector. (See Figure 10.) The other four will be ready by the summer of 1982 , when they will be installed.

Cosmic ray tests of the modules, operated in the limited Geiger mode at about $1.2 \mathrm{kV}$ with argonethylbromide (3-4\%) gas, showed that they were working very well. The signal response of a single channel showed very clear, digitized steps, cha:dcteristic of the Geiger mode operation. The signals were vcry clean, with very low noise. The pulse heights in the anode : nd two cathode strips are tightly correlated, which will simplify eventual separation of electromagnetic showers in a chamber.

\section{Superconducting Magnet Coil}

During 1981 the design for the new TPC superconducting magnet coil was made. It is essentially the same as the design for the previous coil, except that safety factors have been increased. Insulation is improved, since insulation failure was the cause of the accident to the previous coil. In addition, voltages needed for quench protection have been reduced, and packaging is more compact to reduce cracking of insulation. In 1982 the coil will be rebuilt using clean-room procedures. The coil is expected to be tested in December 1982, with installation in PEP-4 during the shutdown of summer 1983 .

\section{Installation of PEP-4 in PEP Beam}

On January 6, 1982, PEF-4 was rolled into the PEP beamline in IR2. The detector started taking data with the beam in February 1982 when the PEP run cycle began. It was found that the TPC worked very wel] under yctual beam conditions. The background in the TPC due to the beam was very small, and no large distortions due to positive ion feedback were observed. Events with many charged particles were successfully reconstructed both online and offline. 


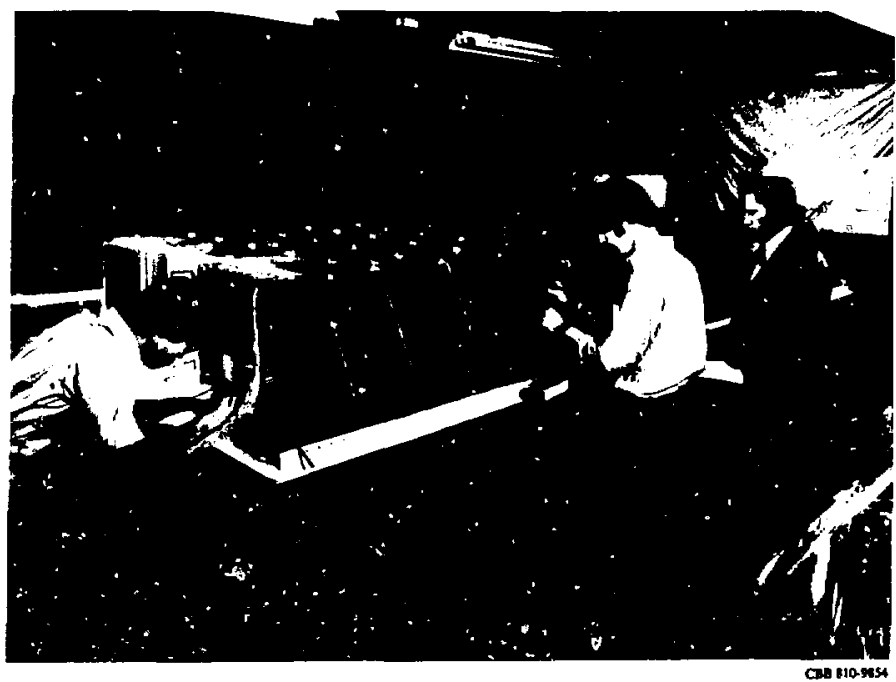

Fig. 10. Wiring one of the bex calorineter modules for PEP-4. Carrying out the long and painstaking task are (left to right) Hiro Aibara, Bill Moses and Tim Nuzum. The calorimeter construction was the joint responsibility of the groups from LBL and the University of Tokyo.

\section{Research Staff}

Hiroaki Aihari, Margaret Alston-Garnjost, Angela Barbaro-Galtieri, Alan V. Barnes, Alan Bross, William C. Carithers, Oven Chamberlain, Chaoging Chen, Alan R. Clark, Orin I. Dahl, Kevin A. Derby, Chrisopher T. Day, Philippe H. Eberhard, David L. Fancher, Hirofumi Fujii, Raymond Z. Fuzesy, Bernard Gabioud, John W. Gary, Fre't:rick R. Goozen, Weixin Gu, Nicholas J. Hadley, John M. Hauptman, Burkhard W. Heck, Hiroyuki Iwasaki, Tunyoshi Kamae, Robert W. Kenney, Leroy T. Kerth, Werner O. Koellner, Rjchard R. Kofler, Stewart C. Loken, Georges W, London, Gerald R. Lynch, Ronald J. Madaras, Philip S. Martin, Jay N. Marx, William Moses, Peter Nemethy, David R. Nygren, P. J. Oddone, Barrie Pardoe, M. Pripstein, P. Robrish, Ron?ld R Ross, Forest R. Rouse, David P. Soroka, Gerald L. Schnurmacher, Gillert Shapiro, Marjorie D. Shapjro, Garth W. Smith, Robert G. Smits, David P.Soroka, M. Lynn Stevenson, John D. Taylor, Nobukazu Toge, Harold W. Van Slyke, Edgar T. Whipple, Hiroaki Yamamoto, Masanri Yamauchi, William A. Wenze!.

\section{Publications}

Future Prospects of the TPC Idea. D.R. Nygren, Physica Scripta, Vol. 23, 584-589, 1981.

Gas-Filled Radiation Detectors in High Energy Physics. J.N. Marx. to be published in "Applied Atomic Collision Physics", edited by E. MeDaniel, Academic Press.

The Superconductor to Rebuild the TPC Coil. P.H. Ebethard and M.A. Green. TPC-LBL-BI-I, 12/17/80.

Analogue Trigger for Calorineter. W.A. Wenzel. TPC-LBL-81-2, $1 / 8 / 81$.

Time Projection Chamber (TPC) Solenoid Fault Loeation, Magnetic Studies. M.I. Green. TPC-LBL-81-3, 1/16/81

PEP-4 Report to DOE K. Mirk. TPC-LBL-81-5, 1/29/81.

Measurement of Energy Loss in the TPC. Gerry Lynck. TPC-LSLBi.6. 
Minutes of TPC Executive Board, Decomber 18 19, 1980 at $L B L$. $R$. Madaras, TPC-LBL-81-7, 1/29/81.

Analog Summing for Calorinkter Trigger, Dnatt 2. W.A. Wenzel. TPC-LBL B1+8, 2/10/81.

A Triangular Tube Proportional Wire Chamber System D.H. Badtke, et a: TPC-JHU-81+1, 2/81,

Resistance Measurements and the Resistance Matrix for the TPC Damaged Coil. P. Eberhard, et at. "TPC-LBL-81+9, 2/B1.

Lucation of Shorts in the TPC Magnet by Inductance Measurements. J. DeOlivares, et al. TPC-LBL-81-10, 1/18/81.

Current Evaluation and the Magnet. K. Derby and 3. DeOlivares. TPC-LBL-8)-11, 2/23/81.

Repart to the SLAC Users Meating on the PEP Program. P. Oddonc. TPC-LBL-81-12, 2/20/B1.

TPC - Bus Controller Users Manual. A Clark. TPC-LBL-BI-13, $3 / 5 / 81$.

Sujpronducing Wire Production and Insulation. J. Taylor. TPCLBL-8I-15, 2/81.

PEP-4 Cable Data Base on the VAX Rocommended Datatrieve Pto cedures for lafiomation Retrieval. W. Koellner. TPC-LBL-81-16, $3 / 81$.

Pads Pertout - 1I. W.A Wenzel. TPC-LBL-B1-17, 3/81.

X-ray Aralysis of the TPC Coil. P. Martin and P. DetPierre. TPC. LEL-81-18, 3/12/8l.

Minutes of the TPC Executive Board Meeting, February 12-13, 1981 at LBL R. Madaras. TPC-LBL-81-19, 3/5/81.

TPC Gas Tube Trailer Pressure Containment G.L Schnurmacher. TPC-LBL-81-20, 3/81.

PEP.4 Progress Report Effort at LBL and SLAC. Many Authors. TPC-LBL-81 -21 $23 / 20 / 8$ :

Pad Channel and Wire List Assignments for Cosmic Ray Test. G. Shapiro. TPC-LAL-81 $22,3 / 24 / 81$.

PEP-4 Status Report Talk Given at SLAC DOE Review, J. Marx. TPC-LAL-81-23, 4/2/81.

BINNAMES - A Way to Store Status of Electonic Bins. F. Rouse. TPC-LBL-8:-24, 4/Bi.

How to Add Oneself to the Analysis System. F. Rouse. TPC-LBL $81.25,4 / 81$.

How to Add Oneself to the Alam System. F. Rouse. ThC-LBL-B!$26,4 / 81$.
Tubes Monte Carto: User's Guide. C.T. Day. TPC-LBL-81-27, $4 / 81$.

11/70 LDB Supervisor (How to Use It). B. Pardoe. TPC-LBL-8t-28, $5 / 81$.

Minutes of the TPC Exerulive Board Meting, April 3, 1981 at SLAC. R. Madaras. TPC-LBL-81-29, S/BI.

Space Charge Neutralizaion in the TPC. W.A Wenzel. TPC.LBL, $81=30,5 / 14 / 8$.

Analysis of Temperature Equilibrium in Electical Shorts. J. de Olivares, et al. TPC-LBL-81m31, $1 / 29 / 81$.

Status Report for the May 18 TFC Board Meetiisg. Various Authors. TPC-LBL-8]-32, 5/81.

Supereonducling Wire for Rebuilding. I. Taylor. TPC-LBL-BI-33, $5 / 21 / 81$.

EXB Drift Consequences of Shortening the Superconducting Coil. ML L Stevenson, TPr.4BL-81*34, 6/2/8i.

$\vec{E}_{\mathrm{D}} \times \vec{B}$ Cotrections for the Conventional Coil, Mi.L. Stevenson. Physies Note No. 921 (1981).

The User Task Interface to the Monitoring System Software, J. Caton. TPC-IBL-81-35, 6/81.

The TPC Moniloring System Software. J, Caron. TPC-LBL-81-36, 6/81.

Minules of TPC Board Meeting, May 18, 1981. R, Madaras. TPCLBL-81-37, 6/8/81.

Two.Photon Evenl Generation in GLOBAL Using the Equivalent Photon Approximalion. B. Shen and G.J. VanDalen. TPC-UCR-81-1, $6 / 11 / 81$.

The Pulse Testing of TPC Insulators Both Large and Small Radius. E.C. Hartwig. TPC.LBL $81+38.7 / 7 / 81$.

A Proposed Method of Rearranging the Sector-To-Foed-Thru-Ring Cables to Unscramble the Pad Signals. M.L Stevenson. TPC.LBL $81-39,7 / 16 / 81$.

HEX Cal - LBL-Tokyo. W. Wenzel. TPC-LBL $81-40,7 / 2 ! / 81$,

What Causes the Hooks? D. Nyeren. TPC-LAL-81-4I, $7 / 29 / B 1$.

Charging Time for the TPC Coil. R. Ross. TPC-LBL-81-42, 8/7/81.

Minules of the TPC Executive Roard, July 9, 1981, at LBL R. Midaras. TPC-LBL-81-43, 8/18/81.

Cautions and Suggestions for TPC Motherboard Maintenance. J.L Harton. TRC-LBL-81-44, 8/21/81. 
Estimate of $\vec{E} \times \vec{B}$ Drifts Due to End Effects in the Conventional Doil. J.L Harton. IPC-LBL-81-45, 9/1/81.

Development and Performance of a High Pressure Hydrogen Time Projection Chamber. T.J. Chapin, et al. TPC-LBL-81-46, 9/81.

Minutes of the TPC Executive Board, August 7, 1981, at 1BL TPCLBL-B1-47, $9 / 8 / 81$.

Superconducting Magnet Comrents. P.H. Eberhard. TPC-LBL-81$48,9 / 14 / 81$.

Electron Drift Velocity in $80 \%$. $20 \% \mathrm{CH}_{4}$ from the TPC Cosmic Ray Test. N. Hadley and G. Lynch. TPC-LBL-81-49, 9/14/81.

Talk Given to SLAC - SPC and LBL - SEAC - Status of PEP.4. J Marx. TPC-LBL-81-S0, 9/BI and 10/81.

TPC-Normal Coil. J.C. Sens. TPC-LBL-81-51, 9/21/81.

TPC Resolution Studies, G. Lynch. TPC-LBL-B1-52, 10/13/81.

TPC Electrostatics Calculations: The Relaration Method for Culculating Electrastatios. C.J. VanDalen. TPC-UCR-81-02, 10/12/81.

Heal Deposited by Magnetic Pulses. P. Eberha:d. TPC-LBL-81-53, $10 / 26 / 81$

Status of Software Needed for TPC Cosmic Ray Test. L Galtieri. TPC-LBL-81-54, 10/21/8;

Minutes of the TPC Exccutive Board, Oetober 9, 1981. R. Madaras. TPC-LBL-81-55, 10/29/81.

E $\times$ B Corrections for the Conventional Coil. M.L Stevenson. TPC-LBL- $81-56,11 / 6 / 81$.

Parameters of the Center. P. Eberhard. TPC-LBL-81-57, 11/81.

SLAC Seminar - "States of PEP-4". J. Marx. 'TPC-LBL-81-58, $12 / 3 / 81$.

Triggering the LBL Time Projection Chamber. M. Ronan, et al. TPC-LBL-81-59, 10/81.

Analog Signal Processing for the Time Projection Chamber. R.C. Jared, et al. TPC-LBL-81-60, 10/81.

Low Noise Preamplifiers/Amplifiers for the Time Projection Chamber. D.A Landis, et al. TPC.LDL-81-61, 10/81.

Use of CCDs in the Time Projection Chamber. R.C. Jared, et al. TPC.LBL-81-62, 10/81.

Energy Resolution Versus Catorimeter Depth. J. Hauptman. $10 / 10 / 81$.

Hex Calorimeter Redesign: Shower Calculations. J. Hauptman. $10 / 15 / 81$.
Hexagonal Calorimeter Calibration. J. Haupman. 7/6/80.

Pole Tip Calorimeter Analysis for the November Test. J. Hauptman, 9/25/81.

Aralog Calorimeter Triggers. J. Haupiman and M. Nakamura. $8 / 5 / 80$.

Ground Plane Insulation Failure in the First TPC Superconducting Coil. M.A Green, et al. 7th International Conference on Magnet Technology IEEE Transactions on Magnetios Vol. MAG 17,5 1855 (March 1981). LBL-12326.

Triggering the LBL Time Projection Chamber. M. Ronan, ef al. Presented at the JEEE Nuclear Science Symposium, San Francisco, CA (October 1981) and to be published in the [EEE Transactions on Nuclear Science, Vol. NS-29, No. 1, February 1982. LBL-13427.

Unpretected Quenches in the First TPC Coil. P.H. Eberhard and R.R. Ross. Physics Note No. 915 (1981).

Gas System Operating Manual (TPC). R.W. Kenney.

\section{The Mark II Magnetic Detector}

The SLAC-LBL Mark II dc ector is a collaborative effort among scientists at $\mathrm{LB}_{\mathrm{L}}$, the $\mathrm{B}$. Richter and M. Perl groups at SLAC, ano the group of $R$. Sciwitters at Harvard University. The program during calendar year 1981 iasd five main thrusts:

1. The operacion of the Mark II detector at PEP: initial operation of a "vertex chamber."

2. Analysis of the PEP data: New results included (i) the first measurement of the $\tau$ lepton lifetime, (ii) measurements of single particle inclusive spectra, which, when compared with SPEAR data, showed scaling violations, (iii) studies of baryon and kaon production, (iv) analyses of multi-jet phenomena, and (v) studies of electroweak interference phenomena.

3. The continuing analysis of data taken with the detector at SPEAR during the period March 1978 to June 1979.

4. Study of modifications to the Mark II for use at the SLC.

5. Study for new calorimeter designs.

\section{Operations at PEP}

During the period between January and July 1981, approximately 15,000 inverse nanobarns $\left(\mathrm{nb}^{-1}\right)$ of integrated luminosity were accumulated. These data were taken with the trigger chamber operating inside the drift chamber. During the summer the new "vertex 
chamber," a high resolution drift chamber which allows more precise track measurements, was instailed. This vertex chamber was designed to detect and measure particles with short lifetimes and has a space resolution $₹$ 100 microns. Another $2000 \mathrm{nb}^{-1}$ were accumulated with the first data of the vertex chamber, during December 1981.

The major responsibility for the vertex chamber design and construction came from John Jaros of SLAC with a substantial contribution of LBL personnel. The sstware and track reconstruction techniques which will allow the chamber to measure short lifetimes were developed at LBL.

Figure 11 shows a $\tau$ lepton pair candidate obtained in the December 1981 running. One $\tau$ decays to a single charged particle and one or two neutrinos, the other to charged particles and one neutrino. Figure $11 \mathrm{c}$ illustrates how the experimenters can zoom in on the vertex region. Figure lla shows the event as observed in the entire detector. The inner circle represents the vertex chamber; then comes the drift chamber; the outer octagon represents the liquid argon shower counters. The rectangles represent the timo-offight counters. The number next to them is the approxinate flight time in nanoseconds. Figure $11 \mathrm{~b}$ shows an enlargement of the vertex chamber, the inner circle in Figure 1la. Figure 1lc shows the sense wires (large X) and actual vertex chamber points (small stars) selected is the fit of the tracks at the inner set of wires (atout $10 \mathrm{~cm}$ radius). Figure $: 1 \mathrm{~d}$ shows the vertex region with a $0.5 \mathrm{~cm}$ calibration length as indicated. The approximate beam-beam collision region is also shown.

\section{Analysis of PEP Data}

Elaborated here are a few of the new topics studied in the Mark II detector.

a) The $\tau$ lepton lifetime.

A study of over $100 \tau$ lepton decays to 3 charged pions allowed the first measurement (as opposed to limits available earlier) of the $\tau$ lifetime. The result

$$
\tau_{\tau}=(4.6 \pm 1.9) \times 10^{-13} \mathrm{sec}
$$

is consistent with the value expected: $(2.8 \pm 0.2) \times 10^{-13} \mathrm{sec}$ if the $\tau$ is a sequential heavy lepton similar $t$ the muon.

b) Scaling violations.

A comparison of single particle spectra between the SPEAR energies, $5.2 \mathrm{GeV}$, and the PEP energy, $29 \mathrm{GeV}$, gave clear indication of scaling violations. Here the fact that both measurements were carried out in the same detector-the Mark II -was important in reducing systematic errurs in the comparison. The measurements showed that in the high $x$ regions $\left(x-2 p / E_{x m}\right)$ the quantity $s \frac{d \sigma}{d x}$ which had scaled very well (that is, had remained constant) in the SPEAR energy region (3-7 GeV) now gives definite scaling violations; namely, values at $29 \mathrm{GeV}$ are about a factor 2 lower than corresponding values at $5.2 \mathrm{GeV}$. (Ste Figure 12.)

c) Baryon and kaon production.

Both $\mathrm{A}^{0}$ and $\mathrm{K}^{0}$ are clearly observed in the Mark II detector. These particles with their distinctive Vee signatures are important in tlat they allow definite identification up to the maximum energy. Energy spectra and cross sections have been presented at the 1981 International Sympesium on Lepton and Photon Interactions at High Energies, Bonn, Germany.

Figure 13(a) gives the $\mathrm{K}_{\mathrm{S}}{ }^{0}$ signal observed in the data of spring 1981. The introduction of the vertex chamber gives substantial improvements in the $K^{0}$ and $\Lambda^{0}$ identification and reduces the background to a negligible level, as shown in Figure 13(b).

\section{Analysis of SPEAR Data}

Detailed analyses of data from the SPEAR detector have continued during 1981. Some of the topics covered are:

a) A first observation of semileptonic decays of charmed baryons.

b) A study of meson spectroscopy, and in particular the observation of the $S^{*} \rightarrow \pi^{+} \pi^{-}$in $\psi$ decay.

c) One-virtual-photon annihilation to various hadronic states.

d) A search for $Q=-2 / 3$ quarks.

e) The observation and study of Bose-Einstein statistics effects on multi-pion correlations in two and three pion clusters at the $\psi$ and other SPEAR energies.

f) A search for rare $\tau$ decay modes and the observation of the Cabibbo-suppressed decay mode

$$
\tau \rightarrow K^{-} v_{\tau} \text {. }
$$

In addition the interaction of two virtual photons has now been studied, in particular, two and four-pion production in $2 \gamma$ decays. 


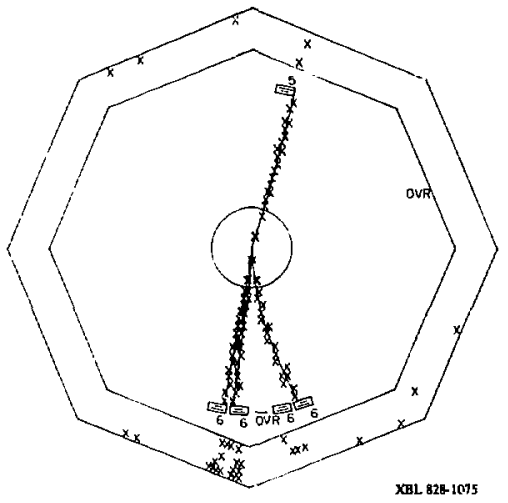

(a) Computer display of the event in the whole detector. Lines emerging from the central region are fits by the computer.

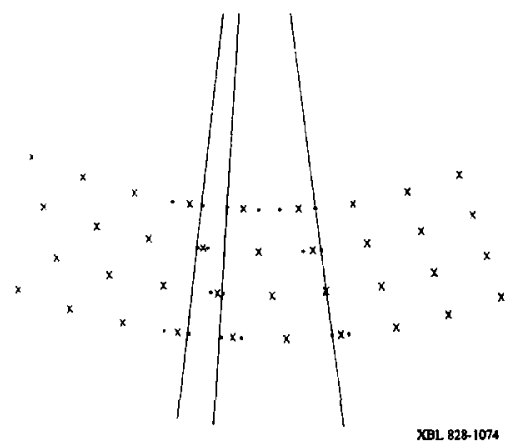

(c) Further enlargement showing the sense wires (X) and the actual points (") selected in the fit of the tracks at the inner set of wi ss of the vertex chamber (at $12 \mathrm{cr}$ 'adius).

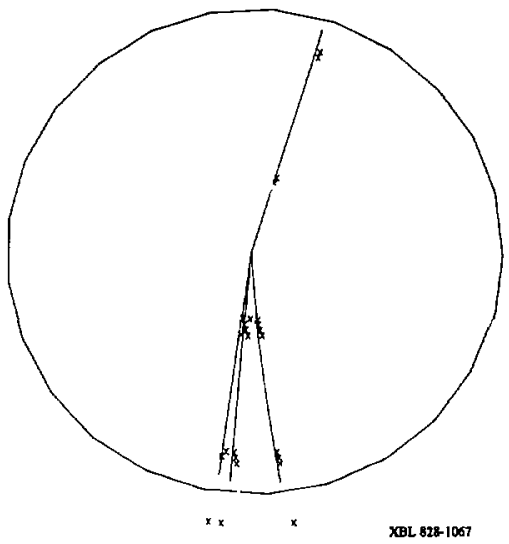

(b) Enlargement of the display, showing only the vertex chamber (the inner circle in (a), approximately $35 \mathrm{~cm}$ in radius).

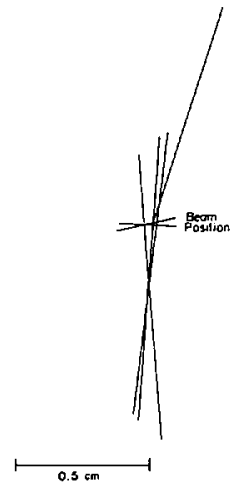

XgL $828-1068$

(d) Detail of vertex region, with fitted tracks and beam position shown. A line 0.5 $\mathrm{cm}$ long is shown to set the scale. With the theoretical lifetime $\tau=2.8 \times 10^{-13} \mathrm{sec}$, the expected mean length of flight is $\gamma \mathrm{ct}=0.068 \mathrm{~cm}$ $=680 \mu \mathrm{m}$.

Fig. 11. A candidate for tau-lepton pair production at $29 \mathrm{GeV}$ in the Mark II magnetic detector (Experiment PEP-5 at IR-12). One tau decays into a single cherged particle plus a neutrino, the other into three charged particles plus a neutrino. 


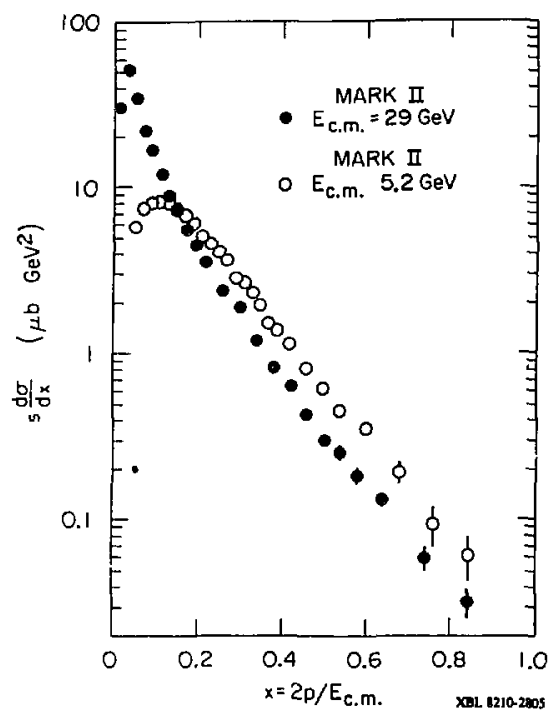

Fig. 12. The so-called scaling inclusive charged particle production cross section $s \frac{d \sigma}{d x}$ versus $\mathrm{x}=2 \mathrm{p} / \mathrm{E}_{\mathrm{cm}}$ for $\mathrm{F}_{\mathrm{kn}}=5.2 \mathrm{GeV}$ and $\mathrm{E}_{\mathrm{con}}=29$ $\mathrm{GeV}$. There is clear evidence for violation of scaling.

Preparations for Use of the Mark II at the SLC

A study is in progress for a proposal to use the Mark II detector as a first detector at the SLC. This study concerns itself with the upgrades needed to make the Mark II compatible with running at the SLC. There is a clear need for a new central tracking drift chamber to measure events having the higher energy and multiplicity that will be encountered at the SLC in $Z^{0}$ mass reăion.

\section{Research Staft}

Gerry Abrams, Dante Amidei, Alain Blondel, William Chinowksy, Pierie Dolpierre, William Dieterle, John Dillon, Mark Eaton, George Gidal, Michael Gold, Gerson Goldhaber, Latrence Golding, A. David Johnson, John Kadyk, Andrew Lankford, Mark Nelson, James Patrick, Peter Rowson, Heidi Schellman, James Strait, George Trilling, Eric Vella, Yunyong Wang, Guozhe Zhao.
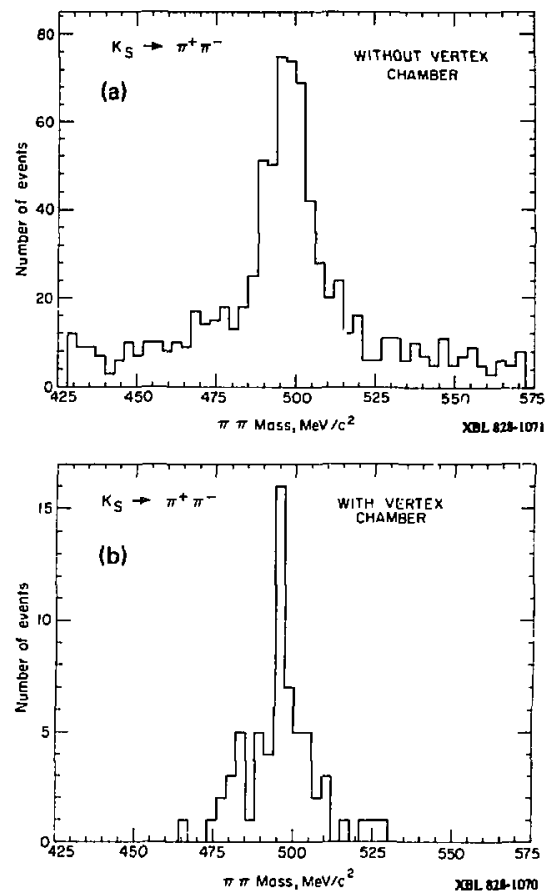

Fig. 13. Two-pion invariant mass distributions of $\mathrm{K}_{\mathrm{a}} \mathrm{C}_{-\pi}{ }^{+} \pi^{-}$candidates i.t the Mark II detector, (a) in Spring ' 81 without the vertex chamber, and (b) in Fail ' 81 with the vertex chamber.

Publications

Observation of the $n_{\mathrm{d}}$ (2980) Produced in the Radiative Decay of the $\checkmark(3684)$. T.M. Himel, G.H. Trilling, G.S. Abrams, M.S. Nam, C.A Blocker, A.P. Blondel, A.M. Boyarsk, M. Brejdenbach, D.L Burke, W.C. Carithers, W. Chinowsky, M.W. Coles, S. Cooper, W.E. Dieterte, J.B. Dillon, J. Doren, boseth, J.M. Dorfan, M.W. Eaton, G.J. Feldman, M.E. B. Franklin, G. Liddal, G. Goldhaber, G. Hanson, K.G. Hayes, D.G. Hitlin, R.J. Hollebxek, W.R. Innes, J.A Jaros, P. Jenni, A. Johnson, J.A Kadyk, A.J. Lankford, R.R. Larsen, M.E. Levi, V. Lith, R.E. Millikan, M.E Nelson, C.Y. Pang, J.F. Patrick, M.L. Perl, B. Riehter, A. Roussarie, D.L Scharre, R.H. Schindler, R.F. Schwitters, J.L Siegrist, J. Strait, H. Taureg, M. Tonutti, E.N. Vella, R.A. Vidal, I. Videau, J.M. Weiss, H. Zacoone Phys. Rev. LetI. 45, 1146 (1980), LBL-1166. 
Direct Pholon Production at the $\psi$. D.L. Scharre, M.S. Alam, AM. boyarski, M. Breidenbach, D.L Burke, J. Dorenbosch, J.M. Dorfan, G.J. Feldman, M.E. B. Franklin, G. Hanson, K.G. Hayes, T. Himel, D.G. Hitlin, R.J. Hollebcek, W.R. Innes, J.A. Jaros, P. Jenni, V. Lith, M.L Perl, B. Richter, R.H. Schindter, R.F. Schwitsers J.L Siegrist, H. Taureg, M. Tonutti, R.A. Vidal, J.M. Weiss, H. Zacoone. G.S. Abrams, C.A. Blocker, W.C. Carithers, M.W. Coles, S. Cooper, W.E. Dieterle, J,B, Dillon, M.W. Eaton, G. Gida], G. Coldhaber, A.D. Johnsan, J.A Kadyk, A.J, Lankford, R.E Millikan, M.E, Nelson. C.Y. Pang. J.F. Patrick. J. Strait, G.H. Trilling. E.N. Vella, and I. Videas. Phys. Rev. D 23, 43 (1981), LBL-10930.

Observation of the Radiative Transition $\psi \rightarrow \gamma E(1420)$. D.L Scharre. G.H. Trilling, G.S. Abrams, M.S. Alam, C.A Blocker, A.M. Boyarski. M. Brcidenbach, D.L Burke, W.C. Carithers, W. Chinowsky, M.W. Coles, S. Cooper, W.E. Dieterle, J.B. Dillon, J. Doreabosch, J.M. Dortan, M.W. Eaton, G.J. Feldman, M.E.B. Franklin, G. Gidal, G. Goldhaber, G. Hanson, K.G. Hayes, T. Himel, D.G. Hitlin, R.J. Hollebeek, W.R. Innes, J.A Jaros, P. Jenni, A.D. Johnson, J.A. Kadyk, A.J. Lankford, R.R, Larsen, V. Lith, R.E. Millikan, M.E. Nelson, C.Y. Pang, J.F. Patrick, M.L Perl, B. Richter, A Roussarie, R.H. Schinḋler, R.F. Schwitters, J.L Siegrist, J. Strait, H. Taureg, M. Tonutti. EN. Vella, R.A. Vidal, I. Videau, J.M. Weiss, and $H$. Zuccone. Phys. Lett 978, 329 (1980). LBL-10931.

Measurement of the Branching Fraction for the Cabibbo-Suppressed Decay K“-(292) $\nu_{r}$ J.M. Dorfan, C.A. Blocker, G.S. Abrams, M.S. Alam, A.M. Boyarski, M. Breidenbach, D.L Burke, w.C. Carithers, W. Chinowsky. M.W. Coles, S. Cooper, W.E. Dieterle, J.B. Dillon, J. Dorenbosch, M.W. Eaton. G.J. Feldman. M.E.B. Franklin. G. Gidal, G. Goldhaber. G. Hanson. K.G. Hayes, T. Himel, D.G. Hitlin, R.J. Hollebeek. W.R. Innes, J.A Jaros, P. Jenni, AD. Johnson, J.A Kadyk, A.J. Lankford, R.R. Larsen, V. Lïh, R.E Millikan, M.E. Nelson, C.Y. Pang, J.F. Patrick., M.L Perl, B. Richter, A Roussaric, D.L Scharre, R.H. Schindler, R.F. Schwitters, J.L Siegrist, i. Strait, H. Taureg, M. Tonutti, G.H. Trilling, E.N. Vella, R.A. Vidal, 1. Videau, J.M. Weiss, and H. Zaccone. Phys. Rev. Let1. 46, 215 (1981). LBL 11394.

Low Energy Weak Interactions and Decays. G.H. Trilling. Rapporteur's Talk, XXth International Conference on High Energy Physics, University of Wisconsin, Madison, WI, July 17-23, 1980. LBL- 11510.

The Properties of Charmed Particles. G.H. Trilling. Phys. Reports 75,57 (1981), LBL- 12283 .

Measurements of the Properties of D Meson Decays. M.S. Alam, A.M. Boyarski, M. Breidenbach, D.L Burke, J. Dorenboseh, J.M. Dorfan, G.J. Feldman, M.E.B. Franklin, G. Hanson, K.G. Hayes, T. Himel, D.G. Hitlin, R.J. Hellebeek, W.R. Innes, J.A Jaros, P. Jenni, R.R. Larsen, V. Lith, M.L Perl, B. Richter, A Roussarie, D.L Scharre, R.F. Schwitters, J.L Siegrist, H. Taureg, M. Tonutti, R.A Vidal, J.M. Weiss, H. Zacoone, G. Abrams, C.A. Blocker, A. Blondel, W.C. Carithers, W. Chinowsky, M.W. Coles, S. Cooper, W.E. Dieterle, J.B. Dillon, M.W. Eaton, G. Gidal, G. Goldhaber, A.D. Johnson, J.A Kadyk, A.J. Lankford, R.E. Millikan, M.E. Nelson, C.Y. Pang, J.F. Patrick, J. Strail, G.H. Trilting, EN. Vella, and I. Videau. Phys. Rev, D 24, 78 (1981), LBL-10905
A Search for Stable Particles Heavier than the Proton and for $\mathbf{Q}-$ -2/3 Quarks Produced in $c^{+} c^{-}$Annihilation, G.S. Abrams, M.S. Alam, C.A Blocker, A Blendel, A.M. Boyarski, M. Breidenbach, D.L Burke, W.C. Carithers, W. Chinowsky, MW. Coles, S. Cooper, W.E. Dieterle, J.B. Dillon, J. Dorenbosch, J.M. Dorfan, M.W. Eaton, G.J. Feldman, ME.B. Franklin, G. Gidal, G. Goldhaber, G. Hanson, K.G. Hayes, T. Himel, D.G. Hitlin, R.J. Hollebeek, W.R. Innes, J.A Jaros, P. Jenni, AD. Johnson, J.A Kadyk. A.J. Lankford, R.R. Larsen, V. Lith, R.E Millikan, M.E Nelson, C. Y. Pang, J.F. Patrick, M.L Perl, B. Richter, A Roussarie, D.L Scharre, R.H. Schindler, R.F. Schwitters, J.L Siezrist, J. Strait, H. Taureg, M. Tonutti, G.H. Trilling. E.N. Vella, R.A Vidal, I. Videau, H. Zaceone. Phys, Lett. 101B, 439 (1981), LBL-122I2.

A Measurement of the Cross Section for Four Pion Production in $\boldsymbol{r}$ Collisions at SPEAR. D.L Burke, G.S. Abrams, M.S. Alam, C.A. Blocker, A Blondel, A.M. Boyarski, M. Breidenbach, w.C. Carithers, W. Chinowsky, M.W. Coles, S. Cooper, W.E Dieterle, J.B. Dillon, J. Dorenbosch, J.M. Dorfan, M.W, Eaton, G.J. Feldnan, M.E.B. Franklin, G. Gidal, G. Goldhaber, G. Hanson, K.G. Hayes, P. Jenni, AD. Johnson, J.A Kadyk A.J. Lankford, R.R. Larsen, M. Levi, V. Lith, R.E. Millikan, M.E Nelson, CY, Pang, J.F. Patrick, M.L Perl, B. Richter, A Roussarie, D.L Scharre, R.H. Schindler, R.F. Schwitters, J.L Siegrist, J. Strail, H. Taureg, M. Tonutti, G.H. Trilline, EN. Vella, R.A. Vidal, I. Videau, J.M. Weiss, and H. Zaccone. Phys. Lett. 103B, 153 (1981), LBL- 12315.

Measurement of the $\tau$ Lifetime. G.J. Feldman, G.H. Trilling, G.S. Abrams, D. Amidei, A. B̈̈cker, C.A. Blocker, A Blondel, AM. Boyasski, M. Breidenbach, D.L Burke, W. Chinousky, M.W. Coles, G. von Dardel, W.E Dieterle, J.B. Dillon, J. Dorenbosch, J.M. Dorfan, M.W. Eaton, M.E.B. Franklin, G. Gidal, L. Gladney, G. Goldhaber, L. Colding. G, Hanson, R.J. Hollebeek, W.R. Innes, J.A. Jaros, A.D. Johnson, I.A Kadyk, A.J. Lankford, C.R. Larsen, B. LeClaire M. Levi, N. Lockyer, B. Lohr, V. Lith, C. Matteuzzi, M.E Nelson, J.F. Patrick, M.L Perl, B. Richter, A Roussaric, D.L Scharre, H. Schellman, D. Schlatter, R.F, Schwitters, J.L Siegrist, J. Strait, R.A Vidal, I. Videau, Y. Wang, J.M. Weiss, M. Werlen, C. Zaiser, and G. Theo. Phys. Rev. Lett. 48, 66 (1982), LBL-13412.

A Study of the Decay $\boldsymbol{r}^{-} \rightarrow \pi^{-} \eta_{r}$. G.S. Abrams, M.S. Alam, A Blondel, A.M. Boyarski, M. Breidenbach, D.L Burke, W.C. Carithers, W. Chinowsky, M.W. Coles, S. Cooper, W.E. Dieterle, J.B. Dillon, J. Dorenbosch, M.w. Eaton, G.J. Feldman, M.E.B. Franklin, G. Gidal, G. Goldhaber, G. Hanson, K.G. Hayes, T. Himel, D.G. Hitlin, R.J. Hollebeek, W.R. Innes, J.A. Jaros, P. Jenni, A.D. Johnson, J.A Kadyk, A.J. Lankford, R.R. Lassen, M. Levi, V. Lïth. R.E. Millikan, M.E. Nelson, C.Y. Pang, J.F. Patrick, M.L. Perl, B. Richter, A Roussarie, D.L Scharre, R.H. Schindler, R.F. Schwitters, J.L Siegrist, J. Strajt, H. Teureg, M. Tonutti, G.H. Trilling. R.A. Vidal, I. Videau, J.M. Weiss, and H. Zaccone. Submitted to Phys. Latt., LBL-13413.

Pion Pair Production in Photon-Photon Collisions at SPEAR. A Roussarie, D.L Burke, G.S. Abrams, M.S. Alam, C.A Blocker, A. Blondel, A.M. Boyarsi: M. Breidenbach, W.C. Carithers, W. Chinowsky, M.W. Coles, S. Cooper, W.E Dieterle, J.B. Dillon, J. Dorenbosch, J.M. Dorfan, M.W. Eaton, G.J. Feldman, M.E.B. Franklin, G. Gidal, G. Goldhaber, G. Hanson, K.G. Hayes, T. Himel, D.G. 
Hitlin, R.J. Hollebeek, W.R. Innes, J.A. Jaros, P. Jenni, A.D. Johnson, J.A Kadyk, A.J. Lankjord, R.R. Larsen, M. Levi, V. Lith, R.E Millikan, M.E Nelson, C.Y. Pang, J.F. Patrick, M.L. Petl, B. Richter, D.L. Scharre, R.H. Schindler, R.F. Schwitters, J.L. Siegrist, J. Strait, H. TaureB, M. Tonutil, G.H. Trilling, EN. Vella, R.A Vidal, t. Videau, J.M Weiss and H. Zacoone. Phys. Lett. 105B, 304 (1981), LBL.13196.

Observation of $S^{*}-\pi^{+}-$in $\psi$ Decay. G. Gidal, G. Goldhaber, j.G. Guy, R.E. Millikan, G.S. Abrams, M.S. Alam, C.A Blocker, A Blondel, A Boyarsk, M. Breidenbach, D.L Burke, W.C. Carithers, W. Chinowsky, M.W. Coles, S. Cooper, W.E Dieterle, J.B. Dillon, J. Dorenbosch, J.M. Dorfan, M.W. Eaton, G.J. Feldman, M.E.B. Franklin, G. Hanson, K.A. Hayes, T. Himel, D.G. Hitlin, R. Hollebeck, W. Innes, J. Jaros, P. Jenni, A.D. Johnson, J.A. Kaulyk, A.J. Lankford, R.R. Lnisen, V. Lith, M.E. Nelson, C.Y. Pang, I.F. Patrick, B. Richter, A Roussarie, D.L Scharre, R.H. Schindler, R.F. Schwitlers, J.L. Siegrist, J. Strait, H. Taureg, M. Tonutti, G.II. Trilling. E.N. Vella, R.A. Vidal, I. Videau, J.M. Weiss, and H. Zacoone. Phys. Lelt. 107B, 153 (1981), LBL 13239.

Multipion Correlations in $\mathrm{e}^{+} \mathrm{e}^{-}$Annihilation at SPEAR, G. Goldhaber. Presented at European Physical Society International Confererce on High Energy Mysics, Lisbon, Portugal, July 9-15, 1981. LBL-1329].

Baryon Protuction at PEP. G. Goldhaber and J.M. Weiss. Fresented at the Annual Mecting of the Division of Particles and Fields of the APS. Santa Cruz. California, September 8-11, 1981. LBL-13360.

Study of the Reaction $e^{+} e^{-\rightarrow \gamma}$ at 29 GeV. G.S. Abrams, LJ. Golding, D.E. Amidei, A. Bäcker, C.A. Blocker, A Blondel, A.M. Boyarsk, M. Breicenbach, D.L. Burke, W. Chinowsky, M.W. Coles, G. vonDardel, W.E. Dieterle, J.E. Dillon, J. Dorenbosch, J.M. Dorfan, M.W. Eatan, G.J. Feldman, M.E.B. FrankJin, G. Gida], L Gladney, G. Goldhaber, G. Hanson, R.J. Hollebeek, W.R. Innes, J.A Jaros, A.D. Johnson, J.A Kadyk, A.J. Lankford, R. R. Larsen, B. LeClaire, M.E. Levi, N. Lockyer, B. Lohr, V. Lith, C. Matteuzzi, M.E. Nelson, J.F. Patrick, M.L. Perl, B. Richter, A. Roussarie, D.L. Scharre, H.M. Schellman, D. Schlatter, R.F. Schwitters, J.L Siegrist, J. Strait, G.H. Trilling, R.A. Vidal, I. Videau, Y. Wang, J.M. Weiss, M. Werlen, C. Zaiser, and G. Zhao. LBL-13493.

Hudron Production by $\mathrm{c}^{+} \mathrm{c}^{-}$Arnihilation at Center-of-Mass Energies Between 2.6 and $7.8 \mathrm{GcV}$. Purt II. Jet Structure and Related Inclusive Distributions. G. Hanson, M.S. Alam, A.M. Boyarsk, M. Breidenbach, F. Bulos, J.? Dakin, J.M. Dorfan, G.J. Feldman, B. Jean-Marie, R.R. Larsen, V. Lith, H.L Lynch, D. Lyon, C.C. Morehousc, J.M. Patersion, M.L. Perl, I. Peruzzi, M. Piccolo, T.P. Pun, P. Rapidis, B. Richter. R.H. Schindler, R.F. Schwitters, J.L Siegrist, W. Tanenbaum, F. Yannucci. G.S. Abrams, D. Briggs, W.C. Carithers, W. Chinowsky, S. Cooper, R.G. DeVoe, C.E. Friedberg, G. Goldhaber, R.J. Hollebeek, A.D. Johnson, J.A. Kadyk, A.M. Litke, R.J. Modaras, H.K. Nguyen, F.M. Pierre, B. Sadoulet, G.H. Trilling, J.S. Whitaker, F.C. Winkclmann, and J.E Wiss. Submitted to Phys. Rev. D. LBLI38B7.

Part 1. Tota! Cross Section Multiplicities and Inclusive Momentum Disuributions. G. Hanson, MS. Alam, AM. Boyarsk, M. Breidenbach, F. Bulos, J.T. Dokin, J.M. Dorfan, G.J. Feldman, G.E. Fisher,
D. Fryberger, D.L. Hertili, J.A Jaros, B. Jean-Marie, R.R. Larsen, V. Lith, H.L. Lynch, D. Lyon, C.C. Morehouse, J.M. Paterson, M.L. Perl, I. Peruzzi, M. Piccolo, T.P. Pun, P. Rapidis, B. Richter, R.H. Schindler, P.F. Scbwitters, J.L. Siegrist, W. Tanenbaum, F. Varnucci, G.S. Abrams, D. Briggs, W.C. Carithers, W. Chinowsky, S. Cooper, R.G. DeVoe, C.E. Friedberg, G. Goldhaber, R.J. Hollebeek, A.D. Johnson, J.A. Kadyk, A.M. Litke, R.J. Madaras, H.K. Nguyen, F.M. Piare, B. Sadoulet, G.H. Trilling, J.S. Whitaker, F.C. Winkelmann, and J.E. Wiss. Phys. Rev. D, LBL-13464.

Measurement of Energy Correlations in $e^{+} e^{-\rightarrow}$ Hedrons. D. Schlatter, G.S. Abrams, D. Amidei, \& Bäcker, C.A. Blocker, A Blondel, A.M. Boyarski, M. Breidenbach, D.L. Burke, W. Chinowsky, M.W. Coles, G. von Dardet W.E. Dieterle, J.B. Dillon, J. Dorenbosch, J.M. Dorfan, M. w. Eaton, G.J. Feldman, M.E.B. Franklin, G. Gidal, L Glndney, G. Goldhaber, L Golding, G. Hanson, R.J. Hollebeek, W.R. Innes, J.A. Jaros, A.D. Johnson, J.A. Kadyk, A.J. Lankford, R.R. Larsen, B. LeClaire, M. Levi, N. Lockyer, V. Lith, C. Matteuzzi, M.E. Nelson, J.F. Patrick, M.L. Perl, B. Richter, A Kouszarie, T. Schaad, D.L. Scharre, H. Schellman, R.F. Schwitters, J.L Siegrish, J. Strait, G.H. Trilling, R.A Vidal, I. Videau, Y. Wang, J.M. Weiss, M. Worien, J.M. Yelton, C. Zaiser, and G. Zhao, To be subritted to Phys. Rev. Lett. LBL-13599.

Meson Spectroscopy Using Hadronic Psi Decays. Invited Talk at New York APS Meeting. G. Gidal. Bull. Am Phys. Soc. Yol. 26, No. 1, 47 (1981).

Proposed Parameterization of the Measurement Capabilities of Electromognetic Calorimeters. J.M Hauptman. SLC-27, 6/22/81.

Can the Decay of Tau to Rho + Neutrino be Measured Directly? J.M. Hauptman. SLC. $37,8 / 25 / 81$.

An Azimuthally Symmetric. Induction Field Drift Chamber as a Vertex Detector. J.M. Hauptman. SLC-52, 10/17/81.

Report of the Subgroup on Calorimeter Geometries. J.M. Hauptman, Chairman. To be included in the procsedings of the Workshop, $10 / 5 / 81$.

A Proportional Wire Calorimeter with Both Strip and Tower Geometries for the SLC. J.M. Hauplman, $7 / 7 / 81$.

\section{Free Quark Search}

During the first six months of 1981 the experimental phase of the Free Quark Search was accomplished, and $17000 \mathrm{nb}^{-1}$ of luminosity were collected. Following the summer shutdown of PEP the detector was used to collect two months of data on cosmic rays.

Analysis was carried out concurrently with the collection of data. To date no quarks have been found, and new limits on quark production have been set in both $\mathrm{e}^{+} \mathrm{e}^{-}$collisions and in cosmic rays. Papers reporting the preliminary analysis were presented at the Washington APS meeting, the EPS conference in Lisbon and the SLAC and Bonn conferences in $\mathrm{e}^{+} \mathrm{e}^{-}$physics. 
Research Staff

Denyse Chew, Robert Ely, Sherwood Parker, Ting Pun.

\section{PEP Experimental Facilities Coordination}

During 1981 the PEP experimental coordination involved the following activities:

1) Coordination of the continuing development of the support systems for PEP experiments, such as online computers, real time links to the central computers, refrigeration plants for superconducting magnets, improvement of masks for backgrouind control, and development of new vacuum pipes.

2) Liaison between the users of PEP and the engineering and physics personnel operating the machine.

Search for Fractionally Charged Cosmic Rays. J. Napolitano, D. Besset. D. Chew, R.P. Ely, S.J. Freedman, R.Fries, B. Gobbi, W. Guryn. F.A. Harris. 1. Karliner, AM. Litke, A Marini, D.H. Miller, S.1. Parker, I. Peruzzi, M. Picoolo, T.P. Pun, F. Ronga, M.C. Ross, V. Vuillemin, T.C. Wang, and D. E. Yount. Absiract submitted to the Spring APS Mecting. Baltimore, MD, April 20-23, 1981, Bull. Avn. Phys. Soc. 26, 584 (1981).

Search for Fractionally Charged Particles al PEP. M.C. Ross, D. Besset, D. Chew, R.P. Ely, S.J. Frocdman, R.Fries, B. Gobbj, W, Guryn, F.A. Harris, I. Karliner, A_M. Litke, A Marini, D.H. Miller, J. Napolitano, S.l. Parker, I. Peruzzi, M. Piccolo, T.P. Pun, F. Ronga. V. Vuillemin, T.C. Wang, and D. E. Yount. Ahstract submitted to the Spring APS Mecting, Baltimorc, MD, April 20-23, 1981, Bul1. Am. Phys. Soc. 26. 630 (1981).

Performance of the Thin Multi-Wire Proportional Chambers of the Free Quark Search Detector at PEP. W. Guryn, R. Fises, S. Parker, D. Yount, D. Besset, D. Chew, R.P. Ely, S. Froodman, B. Gobbi, F.A Harris, I. Karliner, AM. Litke, A Marini, D.H. Miller, J. Napolitano، I. Peruzzi, M. Piccolo, T.P. Pun, F, Ronga, M.C. Ross, V. Vuitlemin, and T.C. Wang. Abstract submitted to the Spring APS Meeting, Baltimore, MD, April 20-23, 1981, Bull. Am Phys. Soc. 26, 630 (1981).

A Scarch for Fractionally Charged Particles at PEP. A. Mariai, I. Peruzzi, M. Piocolo, F. Ronga, D. Chew, R.P. Ely, T.P. Pun, V. Vuillemin, R. Fries, B. Gobbi, W. Guryn, D.H. Miller, M.C. Ross, S.F. Fredmen, AM. Litke, J. Napolitano, T.C. Wang, F.A. Harris, 1. Karliner, S.L. Parker, and D.E. Yount. Presented at the International Conference on High Energy Physies, Lisbon, July 9-15, 1981; invited talk at the International Lepton-Photon Meeting, Bonn, August 1981 (to appear in the proceedings).

A Free Quark Search at PEP. A Morini, I. Peruzzi, M. Piccolo, F. Ronga, F. Harris, I. Karliner, S.I. Parker, D.E. Yount, D.M. Chew, R.P. Ely, T.P. Pun, V. Vuillemin, D. Besset, S. Freedman, A Litke, J. Napolitano, T. Wang, R. Fries, B. Gobbi, W. Guryn, D. Miller, and $M$. Ross. Presented at the SLAC Summer Institute on Particle Physics, July-August, 1981 (to apptar in the proceedings).
3) Evaluation of the impact of PEP proposals on equipment funds and operating resources of the Laboratory

4) Negotiation of agreements with the proponents on the detailed responsibilities of the participating institutions and on the distribution of funds for the arproved experiments.

5) Monitoring of the fabrication and the costs and commitments of detector projects.

6) Long range planning for the utilization of PEP, including funding projections to the agency.

At the beginning of FY 1982 the Time Projection Chamber was installed at PEP, completing the development of the initial phase of PEP detectors. A few months earlier the High Resolution Spectrometer had replaced the first completed experiment at PEP, the Free Quark Search, A total of seven detectors are thus operating at PEP: the Mark II, the TPC, the $2 \gamma$ detector, the Magnetic Calorimeter, the High Resolutioi Spectrometer, DELCO, and the Monopole Search. As these detectors collect data a variety of upgrades are proposed to improve the efficiency of operation and to expand the range of physics covered.

\section{Research Staff}

P. Nemeihy, P. Oddone.

\section{Publications}

Experimental Arens, the PEP Experience P. Nernethy. Isabelle, Proceedings of the 1981 Summer Workshop; edited by A Gordon, pp. 868-878. N.T.I.S. (1981). 
During 1981 work on Fermilab experiments consisted of either research and development toward the future or analysis and publication of data taken in earlier years. In the latter category are the inelastic muen scattering experiment of LBL, Fermilab, and Princeton, in its nearly final stages of analysis, and the inclusive $\pi^{0}$ production experiment of LBL, Cal Tech and MSU. Design, development, anó prototype construction began on the highly segmented end-plug hadron calorimeter for the CDF at Fermilab. Conceptual studies were also initiated on the secondgeneration inclastic muon spectrometer experiment approved for the new muon beam at TeV II. Finally, planning and prelimina-v work occurred on the LBL parts of the first round of experiments planned at the Tevatron using polarized proton and antiproton beams for lambda and antilambda decay.

\section{Hadron Calorimeter for CDF Collaboration}

Design studies for a highly segmented hadron calorimeter for the end plug region of the FNAL Collider Detector Facility (CDF) were initiated as described in the CDF Design Report.

Research on 2 new mode of saturated gas gain (radial streamer mode) has begun. This mode may be well suited to hadron calcitimetry. A shower counter prototype operating in the semi-proportional gas gain region was tested at SLAC.

A full scale prototype of one section of the CDF

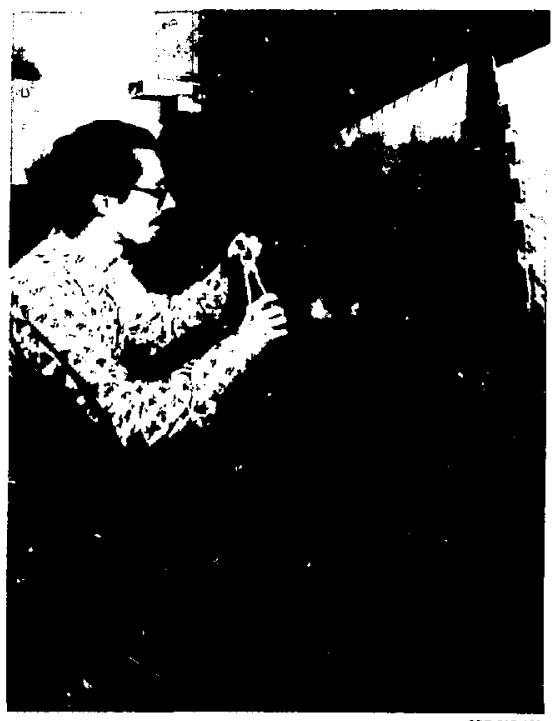

CBA 127.323$)$

Fig. 14. Bill Baldock of the LBL Assembly Shop working on a section of the CDF end plug hadron calorimeter. end plug hadron calorimeter (equaling $1 / 24$ of the full system) is being constructed. The device utilizes resistive plastic tubes with square cross section. This prototype will be tested in the FNAL M4 beam. (See Fig. 14.)

\section{Research Staff}

William Carithers, William Chinowsky, Robert Ely, Michael Gold, Robert Kelly, Peter Rowsen, Kirk Siinsky.

\section{Muon Reactions}

The Fermilab Muon Experiment is a collaboration between LBL Fermilab and Princeton University. Analysis of data on single and multimuon final states continued through 1981 and results were reported at conferences and published.

The study of three-muon final states provided information on elastic and inelastic production of $\psi(3100$ ) by muons. (See Fig. 15.) The data indicate that the first order QCD perturbative calculation, photon-gluon fusion, describes elastic production in most respects but that the $Q^{2}$ dependence is steeper than the model predicts. Inelastic $\psi$ production is adequately handled by expanding the calculations to second order where final state gluons provide the observed energy. The cross section predicted by these calculations is, however, significantly less than the measured value.

The study of "exotic" events containing add charge combinations or more than three final state muons has been completed. From a sample of sixty exotic events, a single odd-sign $3 \mu$ event (and also single $4 \mu$ and $5 \mu$ events) with unusual kinematics have been identified. Unfortunately, ro compelling evidence for new physics has emerged. (See Fig. 16.)

Work continued on the study of inelastic muon scattering and the determination of nucleon structure functions. This analysis will be completed in the near future. 


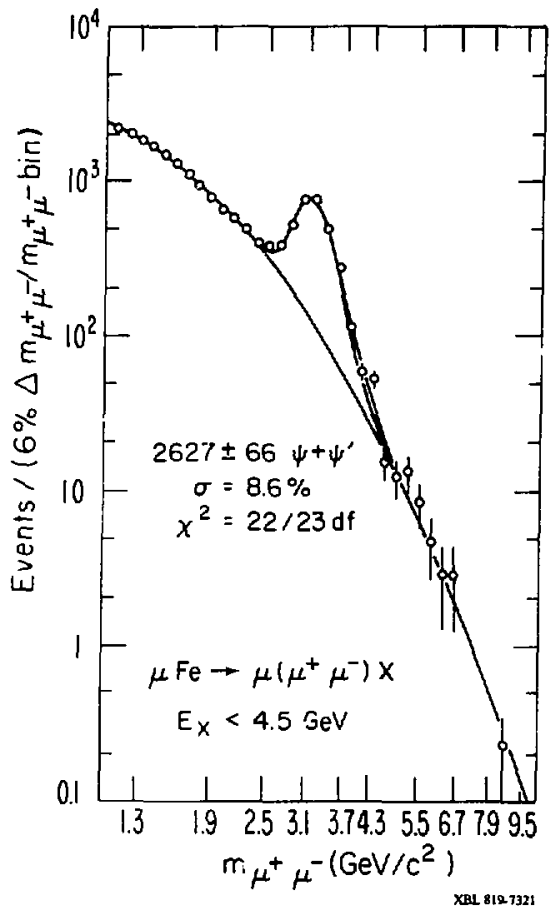

Fig. 15. Muon pair invariant mass distribution for masses above $1.12 \mathrm{GeV} / \mathrm{c}^{2}$ for the final sample of events from $\mu \mathrm{Fe} \rightarrow \mu\left(\mu^{+} \mu \rightarrow\right) \mathrm{X}$ observed in the Fermilab Muon Experiment (E-203A). The peak at $3-4 \mathrm{GeV} / \mathrm{c}^{2}$ is the sum of $\psi\left(3.1 \mathrm{GeV} / \mathrm{c}^{2}\right)$ and $\psi\left(3.7 \mathrm{GeV} / \mathrm{c}^{2}\right)$ production.

\section{Research Staff}

Alan R. Clark, Frederick R. Goozen, Karl J. Johnson, Leroy T. Kerth, Stewart C. Loken, Thomas W. Markiewicz, Peter D. Meyers, Timothy E. Nuzum, Wesley H. Smith, Mark W. Strovink, Thomas F. Weber, William A. Wenzel.

Publications

Charm Production by Muons and its Role in Scale-Noninvariance. G.D Gollın, F C. Schoemaker, P. Surko. A R. Clark. KJ. Johnson,
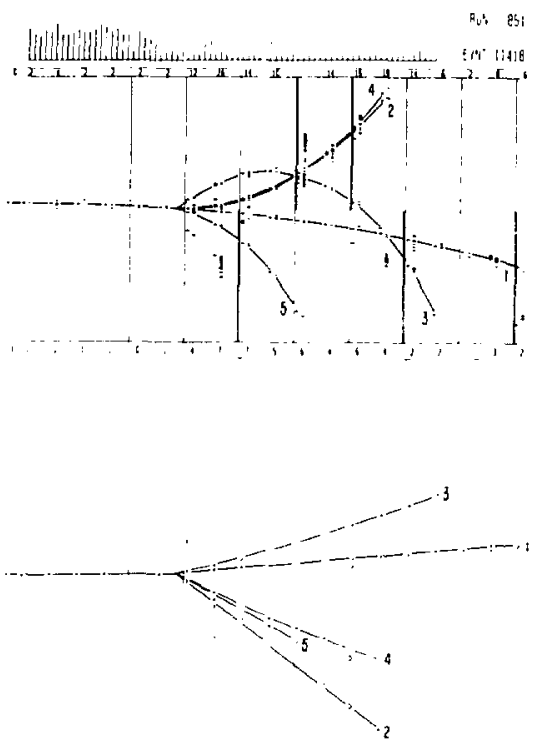

Fig. 16. Computer display of an example of exotic multi-muon production in the muon spectrometer at Fermilab. (XBL 814-2256)

L. T. Kerth, S. C. Loken, T. W. Markitwicz, P. D. Meyers, W. H. Smith, M. Strovink, W. A Wenzel, R. P. Johnson, C. Moore, M. Mugge, R. E. Shafer. Phys. Rev, D24, 559 (1981).

Siudy of Rare Processes Induced by 209-GeV Muons. W.H. Smith. Ph.D. thesis, LBL-12789, May 1981.

Elastic and Inelastic $\psi$ Production by Muons. S.C. Loken. Presented at the XVIth Recontre de Moriond, Elementary Particle Physies Mect* ings, Les Arcs, Savoie, France, March 15-27, 1981. LBL 12814, June 1981.

Review of Multimuon Production by Muons. M. Strovink. Invited talk presented at the 198I International Symposium on Lepton and Photon Interactions at High Energies, Bonn, West Germany, August 2423, 1981, and at Particles and Fields. American Physical Society Conference, Santa Cruz, CA, September 9-11, 1981. LBL-13478, October 1981.

Study of Rare Processes Induced by $209-\mathrm{GrV}$ Muons. W. H. Sinith, A. R. Clark, K. J. Johnson, L T. Kerth, S. C. Loken, T, W, Markiewicz, P. D. Meners M. Strovink, W. A. Wenzel, R. P. Johnson, C. 
Moote, M. Mugge, R. E Shafer, G. D. Colln, F. C Shoemaker, and P. Surko. LBL-13422 Preprint, October 1981, submitted to Physical Review D.

\section{Tevatron Muon Program}

Fermilab will build a new muon beam for use with the Tevatron. The Berkeley-Fermilab-Princeton collaboration has been approved to move the Multimuon Spectrometer to the new muon beam. The experiment will provide data on single and mu!timuon final states at incident muon energics up to $700 \mathrm{GeV}$.

The study of inelastic muon scattering will extend measurements of nucleon structure functions up to a momentum transfer squared $\left(Q^{2}\right)$ of $1000[\mathrm{GeV} / \mathrm{c}]^{2}$. These data will provide important tests of Quantum Chromodynamics.

The study of multimuon events is expected to provide information on the dynamics of heavy quark production and a measurement of high mass vector meson production. In addition, the sample of exotic events will be significantly increased.

\section{Research Staff}

Alan V. Barnes, Alan R. Clark, Frederick R Goozen, Karl J. Johnson, Leroy T. Kerth, Stewart C. Loken, Timothy E. Nuzum, Thomas F. Weber, William A. Wenzel.

\section{Sirong Interaction Experiments at FNAL}

Our latest FNAL experiment (E-350) investigated the reaction $\pi-p \rightarrow \pi^{0} X$ in the forward direction as a test of the triple-Regge formalism. The $200 \mathrm{GeV}$ data are in the tinal stages of analysis.

A discrepancy at small $|t|$ between the $p$ trajectory derived from this work and the $\rho$ trajectory deduced from our previous $\pi^{-} p \rightarrow \pi^{0} \mathrm{n}$ data in the same energy range has led to a further check of the tripleRegge model. A comparison of inclusive kaon and inclusive pion scattering in the triple-Regge region shows that the $\mathrm{SU}(3)$ relation $\sigma\left(\mathrm{K}^{-} \mathrm{p} \rightarrow \mathbf{K}^{0} \mathrm{X}\right)=$ $0.5\left(\sigma\left(\pi^{-} p \rightarrow \pi^{0} X\right)+2.2 \sigma\left(\pi^{-} \rightarrow \eta X\right)\right)$ is only approximately valid in the kinematic range of $x \geq 0.7$ and $-t$ $\leq \mathrm{l}(\mathrm{GeV} / \mathrm{c})^{2}$ for the final state particle. The observed SU(3) breaking corresponds (in Regge formalism) to the ratio of the $K \bar{K}$ to $\pi \pi(\eta)$ couplings of the $\rho$ and $A_{2}$ trajectories being $14 \%$ larger than SU(3) predictions. A paper is ready for submission to Phys. Rev. on this subject.

A summary article on our triple-Regge experiments at FNAL has recently appeared. There remains cne final article to be written on the entire set of exper- iments on $\pi^{-p}$ elastic and $\pi^{-p}$ triple-Regge (nearly elastic) results. It should be forthcoming this year.

\section{Research Staff}

O. Dahl, R. Johnson, R. W. Kenney, M. Pripstein

Publications

An SU(3)-Based Comparison Between Inclusive Knon and Pion Scattering in the Triple Regge Region. A.V. Barnes, G. C. Fox, R. G. Kennett, R. L. Walker, P. M. Yager, H. G. E. Kobrak, R. E. Pitt, R. A Swanson, K W. Edwards, M. Harrison. O. I. Dahl, R. W. Kenney, A. Ogawa, M. Prin, M. A Abolins, W. R. Francis, D. P. Owen. Abstract, CALT-68-886. Submitted for publication December 198i.

Inclusive $\pi^{0}$ and $\eta$ Production from Kaon, Proton and Anti-Proton Beams in the Triple-Rege Region. R.E Kennet, A. V. Barnes, G. C. Fox, R. L. Walker, O. I. Dahl, R. W. Kenney, A Ogawa, M. Pripstein. Nucl. Phys. B177, 1 (1981).

\section{Experiments Using Polarized Proton and Antiproton Beams}

Lambda and anti-lambda production at Tevatron energies is so abundant that enriched polarized proton, and polarized antiproton beams from lambda and antilambda decays will be built for counter physics as part of the Tevation II development at FNAL A first round of experiments has been approved to make the first use of the polarized beam. Measurements will include total cross-section differences with polarized beam and target, high-x low- $\mathrm{p}_{\mathrm{T}}$ inclusive pion production with polarized beam, lambda production with polkrized beam on unpolarized hydrogen target, and inclusive $\pi^{0}$-production under the same conditions.

This will be a collaborative effort, involving physicists from Argonne National Laboratory, Rice and Northwestern Universities; LAPP, Annecy, France; INFN, Sezione di Trieste, Italy; Serpukhov and Kyoto, as well as LBL.

\section{Research Staff}

G. Shapiro.

Publications

Physies with a High Energy Polarized Proton Beam at Fermilab. I. P. Auer, E. Colton, R. Ditzler, D. Hill, H. Spinka, G. Thoodosiou, K. 
Tostiaka, D. Underwood, R. Wagner, A Yokosaws, Y. Hemmi, R. Kikuchi, K.Miyaks. T. Nakamura, N, Tamura, R. Birsa, F. Bradamante, M. Giorgi, A Penzo, P. Schiavon, A Villari, K. Kuroda, A Michalowicz, D. Perrel-Gallix, G. Shspiro, H, E. Miettinen, T. A
Mulera, G. S. Mutchler, G. C. Phillips, J. B. Roberts. High-Energy Physics with Polarized Beams and Pnlarized Targets: Proceedings of the 1980 International Symposium, Lausanne, September 25-October 1, 1980, C. Joseph and J. Soffer, eds, (Basel 1981).

\section{Search for Effects of a Right-Handed Gauge Boson}

Experiment 185, a search for right-handed currents in $\mu^{+}$decay, is well underway at TRIUMF after proposal approval in November 1980. The data will be the first to measure precisely the endpoint decay spectrum for muons with extremely high ( $>0.995$ ) average polarization.

The experiment is provoked by the apparent absence of charged weak currents which do not possess the conventional (V-A) left-handed helicity. Electroweak gauge theories beyond the Glashow-WeinbergSalam "standard model," and most Grand Unified theories, instcrid tend to prefer a description of nature which is left-right symmetric.

Two parameters describe the phenomenology of right-handed charged weak currents: the mass $\mathbf{M}_{R}$ of the right-handed weak boson $W_{R}$ which mediates the current, and the mixing angle $\zeta$ which measures the extent to which the mass eigenstates of the $W_{L}-W_{R}$ system are not parity eigenstates. These parameters are significantly constrained by $(A)$ the $\xi$ parameter and $(B)$ the $\rho$ parameter in $\mu$ decay; (C) the electron polarization in Gamow-Teller $\beta$ decay; (D) the asymmetry parameter in ${ }^{19} \mathrm{NE} \beta$ decay; (E) calculation of the $\mathrm{K}_{\mathrm{L}}-\mathrm{K}_{\mathrm{S}}$ mass difference assuming the electroweak gauge group $\mathrm{SU}(2)_{\mathrm{L}} \times \mathrm{SU}(2)_{\mathrm{R}} \times \mathrm{U}(1)$; (F) limits on neutrinoless double $\beta$ decay. Experiment 185 measures the $\xi$ parameter (A), which is sensitive both to $\mathbf{M}_{R}$ and to $\zeta$ : a null result would limit $M_{R}>600 \mathrm{GeV} / \mathrm{c}^{2}$ and $|5|<0.03$ independently of each other. In terms of measured quantities, these limits would surpass by more than one order of magnitude the existing data on (A), (B), and (C). Constraints (B) through (F) are sensitive to $M_{R}$ and $\zeta$ only in particular combinations. While very precise, constraints (E) and (F) are modeldependent, and (E) is insensitive to 5; method (F) limits $M_{R}$ only in combination with lepton nonconservation.

Construction and installation of the Muon Polarimetcr is complete. (See Fig. 17.) One of its two LBL magnets, designed especially for the experiment, is powered by four independent coils, controlling the degree of stopped muon polarization and range of decay positron angular acceptance. The drift chamber system includes 120 planes of wire anodes and cathodes. (See Fig. 18.) Exclusive of $\mathrm{He}$ in the target flask and scintillators at the rear, the spectrometer presents less than

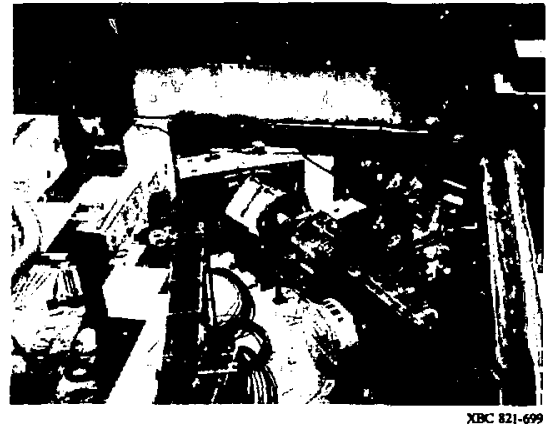

Fig. 17. Overall view of the muon polarimeter system at TRIUMF. Graduate student Alex Jodidio examines a cable. For a plan of the apparatus, see the 1980 Annual Report, p. 31.

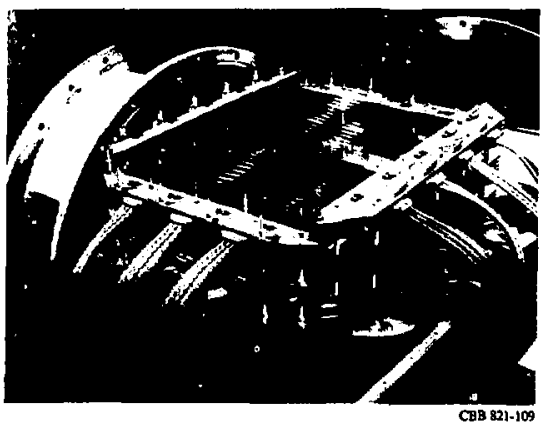

Fig. 18. Wire drift chamber ready for assembly as a positron detector. The "bass drum" chamber can be seen in position at the side of the Sagane magnet in Fig. 17. 
$200 \mathrm{mg} / \mathrm{cm}^{2}$ to the combination of incoming $\mu^{+}$and ou going $\mathrm{e}^{+}$. The LBL PDP-11/34 acquires data seliably at an average rate of $2 \times 10^{5}$ bits/second online; it and the offline VAX $11 / 780$ are supplying plentiful monitoring diagnostics.

Data collection will continue in a series of runs during 1982-83 in the M13 channel at TRJUMF.

\section{Research Staff}

John Carr, George Gidal, Bruno Gobbi (Northwestern Univ.), Alex Jodidio, Christopher Oram (Univ. of Brit- ish Columbia/TRIUMF), Kirk Shinsky, Herbert Steiner, David Stoker, Mark Strovinl., Robert Tripp.

Publications

Possible Deviations from (V-A) Charged Currents: Precise Measurement of Muon Decay Parameters. M. Strovink In Weak Interactions as Probes of Unification (Virginia Polytechnic Institute, Dec. 4-6, 1980), G. B. Collins, L N. Chang, and J.R Ficenec (eds.), America, Institute of Physics Conference Procedings No. 72 (AIP. New York, NY. 1981). pp. 46-70.

\section{Particle Data Center}

The Particle Data Center compiles high energy physics data, and makes it available to the physics community in easily usable form. The "Review of Particle Properties" and other publications are distributed to the community periodically, and computer searchable databases are also being made publicly available. Most members of the group are also active in research projects at LBL and elsewhere.

There are five major activities-

1) Compilation if particle properties, and the issuance of the "Review of Particle Properties," with the associated Data Booklet and Pocket Diary.

2) Compilation of descriptive and bibliographic information on published articles, preprints, and current experiments in progress. Publication of indexed guides to current literature (LBL-90) and experiments (LBL-91).

3) Maintenance of a database of compiled high energy physics reaction data. Periodic publication of reports and tapes covering these data.

4) Maintenance and continued development of a computerized database management system to store, maintain, update, retrieve, and display the compiled information referred to above. The systems described in 2), 3), and 4) comprise the "Particle Physics Data System."

5) Participation in various research projects in collaboration with LBL and other research groups.

\section{Data Compilation Efforts}

Although the year was marked by a considerable reduction in staff, there was solid accomplishment:

(a) The annual updating of the data for the 1982 publication of the "Review of Particle Properties" was done. Work commenced on the actual publication itself. The listings of particle properties continued to grow at a rate of about 10 per cent per annum.

(b) The annual edition of the Pocket Diary, with its cecompanying High Energy Physics laboratory Phone and Address List was published.

(c) The annual edition of LBL-91, "Compilation of Current High Energy Physics Experiments," was produced.

(d) A system for entering the encoding done by our Russian collaborators into our databases was implem-nted. The group was visited by N.A. Tyurin and V.V. Ezhela of Serpukhov to discuss the collaboration. A protocol was signed detailing the collaborative agreement between the two labs.

(e) The "EXTRACT" system for easy extraction of data from the reaction data database system was completed and implementer on the $\mathrm{Cal}$ Tech VAX 11/780 operated by the Fox group.

(f) Ail index to data available in the Experimental Reaction Data database, implemented on the 
Cai Tech VAX 11/780, was published as LBL-92 "Compilation of High Energy Physics Reaction Data: Inventory of the Particle Data Group Holdi. gs 1980," (1981).

\section{Participation in Research Projects}

Physicist members of the group carried out rescarch projects with other LBL groups and oulside groups. Trippe worked on an analysis of the consequences oi Bell's theorem. Wohl prepared a study of isospin relations and began a study of more generalized $S^{\prime}(n)$ relations. Yost completed work on a Four-View Geometry Program for a SLAC bubble chamber experiment to study the properties of charmed particles and to do other types of studies. Kelly, who was a member of the group for only part of the time, completed serving as High Energy Physics Section Editor for the $50^{\text {th }}$ Anniversary Handbook of the Amcrican Institute of Physics, worked on the problem of gluon bremsstrahlung in QCD, and worked on theoretical analysis of other problems associateJ with $\mathrm{e}^{+} \mathrm{e}^{-}$collisions. Shimada worked with Kelly before departing for Japan in March.

\section{Research Staff}

Charles P. Horne, Robert L. Kelly, Alan Rittenkerg. Tokuzo Shimada, Thomas G. Trippe. Charles G. Wohl, George P. Yost.

Publications

Compilation of High Energy Physics Reaction Dala: Inventory of the Particle Data Group Holdings 1980. G C. Fox, P. R. Stevens, B. J. Read, F, D. Gault, A. Rittenberg. M. S. Hutchinson, C. P. Horne, R. L Kelly. F. E. Arrstrong, D. R. Richards. T. G. Trippe, G. P. Yosi. R. G. Roberis, R L Crewford. LBL-92 (1981).

Compilation of Current High Energy Physics Experiment. C. G. Wohl. R. L Velly. F. E. Armstrong. C. P. Hurne, M. S. Hutetisison. A Rittenberg, T. G. Trippe, G. P. Yost, L Addis, C. E. W. Ward, N. Bagget, Y. Goldschmidt-Clermont, P. Joos, N. Gelfand, Y. Oyanagi. S. N. Gudtsin. Yu. G. Ryabov. IBL-9l Revised, UC-34C (198I)

Evidence for Hard-Gluon Bremsstrah]ung in a Deep Inelastic Neutrino Seattering Experiment. H. C. Ballagh, H. H. Bingham, W. B. Fretter, T. Lawry, L. R. Lyneh, J.Lys, J. Orte!, M. D. Sokoloft, M. L Stevenson, G. P. Yost, D. Ciec, F. R. Huson, D. J. Miller, E. Schmidi, W. Smart. E. Treadwell, R. J. Cence, F. A Harris, M. D. Jones, A Koide, S. I. Parker, M. W. Peters. V. Z. Pelerson, V. J. Stenger, and G. N. Taylor, T. H. Burnet, H. J. Lubatti, K. Moriyau. D. Kees, G. M. Swider, E. Wolis., U. Camerini, W. Fry. R. J. LoveIess, M. Ngai. D. D Reeder. Phys. Rev. Let?. 47, 596 (1981).
Diffractive Dissociation of Pions in $\pi^{-}$Interactions at $15 \mathrm{GeV} / \mathrm{c}, R$. Harris, L. A. Dunn, H. J. Lubatti, K.Moriyasu, H. H. Bingham, W. B. Fretter, W. R. Graves, F. C. Porter, G. P. Yost. Zeit. Phys. C9, 275 (1981).

AIP 50" Anniversary, Physics Vade Mecum, 1981. R. L. Kelly, section editor Iar High Enetgy Physics.

Photon and $x^{0}$ Production at the FNAL $\vec{p}-p$ Collider. I Hinchlifie and R.L Kelly, LBL-12274, (February 1981).

$\pi N, k N$ and $\bar{K}$ N Phenomenology. R.L. Kelly. Published in Procedings of the Workshop on Nuclear and Particle Physics at Energies up to $31 \mathrm{GeV}$, Los Alamos (198I).

Dilepton Signature in $e^{+} e^{--}+\mathrm{Hl}+l-$ R. L. Kelly and T. Shimada. Phys, Rev. D23, 1940 (1981).

Isospin Relations by Counting. C.G. Wohl. to be published in Am Journal of Physies (May 1981). LBL-12858.

Gluon Bremstrahlung Effects in Large P-transverse Hadron Hadron Seatlering. G.C. Fox and R.L. Kelly, LBL-13985 (1981).

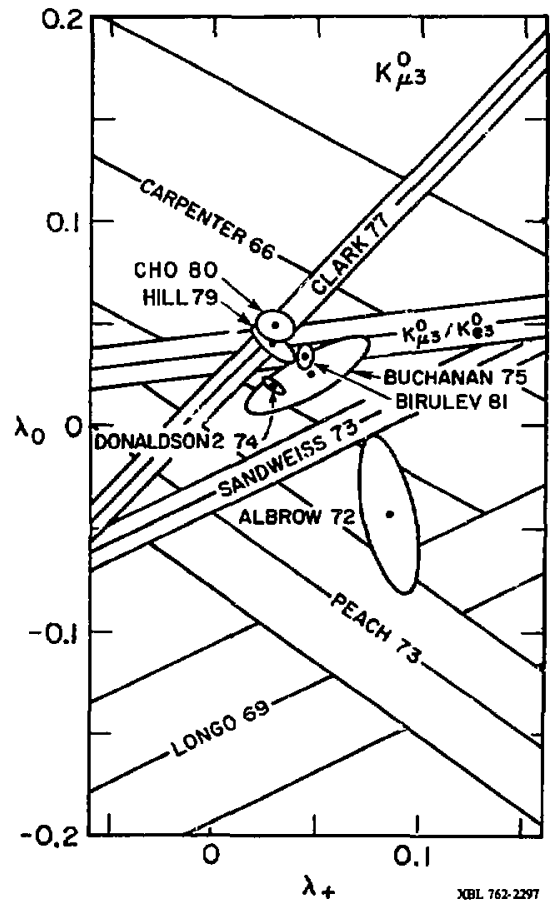




\section{Astrophysics and Earth Sciences}

During 1981 a number of physics-oriented programs in astrophysics and geoscience continued, with funding from both within and without the Laboratory. The "early warning" system of automated searching for new supernovae neared completion, while the detailed study of the anisotropies of th $=$ cosmic background radiation continued. A growing effort, funded by the University of California as a system, was on research and development for the ten-meter telescope. Smaller earth science projects were a study of the $\mathrm{CO}_{2}$ budget in the atmosphere and the development of a very small cyclotron for ultrasensitive detection of long-lived radioisotopes in meteorites and in the atmosphere.

\section{Supernovae Detection and Observation}

One group has built the hardware and is finishing the software for a program to search for and observe supernovae early in their explosions. Supernovae play a fundamental role in many areas of astrophysics. They are the progenitors of black holes, neutron stars, and pulsars; they are probably responsible for nucleosynthesis of all elements heavier than iron; stars, planets, and interstellar grains are made on supernova shockwaves; cosmic-rays are accelerated on the shockwaves either at the explosion itself, in interstellar space, or in the enormous electric fields at the pulsar. The remnants of $\mathrm{SN}$ explosions are strong sources of radio, optical, and $\mathrm{x}$ - and gamma-ray radiation.

Although about 400 supernovae have been reported in the last 6,000 years, only five were seen before maximum light. Calculations indicate substantial differences in the pre-maximum light curve, depending on the details of the explosion; yet this section of the light curve is where there is practically no data. The search should detect one supernova every three to six days at 10 to 40 percent of maximum light before the peak. For supernova explosions in the nearby Virgo cluster of galaxies, one should see supernovae at a few percent of maximum light.

An RCA charge-coupled imaging device (CCD) wil be used with a 36-inch automated optical telescope to repeatedly scan about 7500 galaxies, cycling through this sample every three days. The image of the galaxy will be compared to standard (no supernova present) image of the galaxy, and checked for a brightness increase in any one image element. $A$ dedicated PDP $11 / 44$ mini-computer is used for controlling the $\mathrm{CCD}$ and telescope and calculating the brightness differences. When a supernova is discovered, it will be studied in detail with existing ground-based and orbiting satellites to take optical, U.V., and gamma-ray spectra.

During calendar year 1981 the group finished building and testing the CCD support electronics, and testing and wiring the $\mathrm{CCD}$ dewar, the software for reading the image into the computer and displaying it on a graphics monitor, the servo amplifiers for automatically moving the telescope from galaxy to galaxy, the overall design for much of the crucial software, and snmic of the system support software. The group hopes to detect its first supernova in late summer or early fall of 1982.

\section{Research Staf}

M. Shane Burns, Frank Crawford, George Gibson, Jordin Kare, Rich Muller, Carl Pennypacker, Robbie Smits, Roger Williams, John Yamada.

\section{Publications}

The Berkeley Automated Supernovae Search. J. Kare, C. Pennypacker, R. Muller, T. Mast, F. Crauford, S. Burns, R. Williems. Presented at NATO Conference on Supernovae, 6/29-7/I0/81, Cambridge, England To be published in conference proceedings.

\section{Cosmic Background Radiation Primordial Blackbody Radiation}

\section{Angular Distribution/Anisotrupy}

The experimental program to detect anisotropies in the cosmic background radiation (CBR) has continued. An anisotropy at the $10^{-3}$ level has been measured using a $9 \mathrm{~mm}$ wavelength radiometer flown from the U.S. and Peru aboard a U-2 aircraft. This first order anisotropy is interpreted as due to the cosmological motion of the Milky Way Galaxy.

The group has built and successfully flown a balloon-borne liquid-helium-cooled radiometer to measure the anisotropy of the CBR at the $3 \mathrm{~mm}$ wavelength. (See Fig. 19.) The first flight, in July 1981, suffered from radar and telemetry interference problems, but the flight on 4 November 1981 returned $81 / 2$ hours of data from an altitude of 90,000 feet. The most recent flight, on 27 April 1982, returned aboui 10 hours of data, which is as yet unanalyzed. (See Fig. 20.)

The data, cleariy showing the first order anisotropy, is now being analyzed to characterize the dipole and to search for higher-order anisotropies. (See Fig. 21.) 


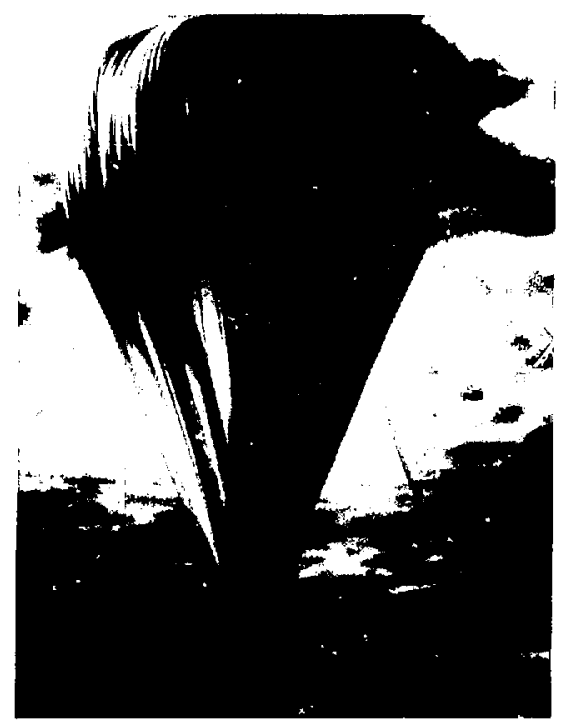

Cas i27-64)

Fig. 19. Balloon carrying liquid helium-cooled radiometer ready for an early morning launch at Palestine, Texas.

The April flight will symmetrize the sky coverage from the 4 November flight. The results from these three flights will be analyzed and published quickly because of the implications on high-frequency spectral distortion and the report of quadrupole anisotropy.

Additional flights of the liquid-helium-cooled 3mm system are planned to complete as much sky coverage as possibie. This probably means at least one additional fight from the U.S. and from the Southern Hemisphere. It is hoped to add another frequency to the payload for direct comparison of measured anisotropy.

The group has continued designing a satelliteborne CBR anisotropy experiment at 13,9,6, and $3 \mathrm{~mm}$ wavelengths. It also has constructed 9 and $3 \mathrm{~mm}$ prototypes and has conducted tests and begun other experiments.

In April 1982 the satellite anisotropy experiment will begin building and testing engineering units of the radiometers and subassemblies of the space flight system.

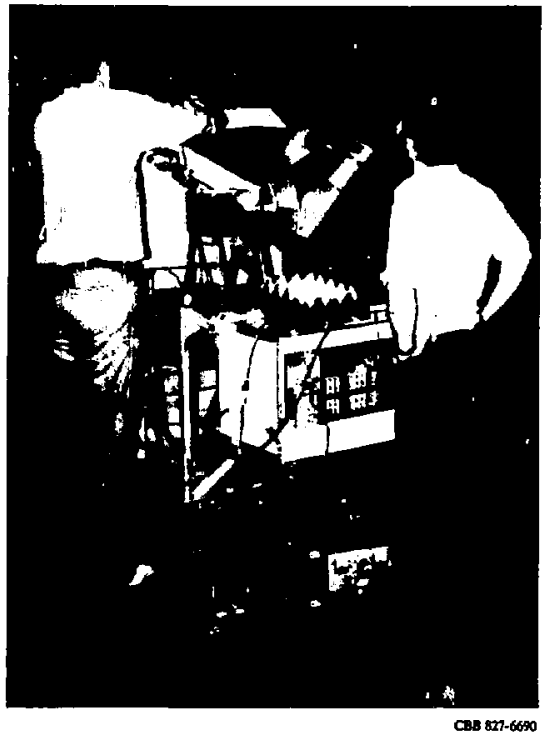

Fig. 20. Pre-dawn final adjustment of radiometer instrument package by George Smoot (left) and Phil Lubin.

\section{Polarization}

The physicists have made 9-min measurements of the polarization of the CBR and begun measurements at $30 \mathrm{~mm}$. At $9 \mathrm{~mm}$ and $30 \mathrm{~mm}$ no polarization greater than $10^{-4}$ is seen. Measurements of the CBR polarization are continuing at 30 and $9 \mathrm{~mm}$ and will soon begin at $3 \mathrm{~mm}$. For 3 and $0.9 \mathrm{~cm}$ wavelengths the system noise seems to be the limiting factor, so that continued observation improves the measurement and sky survey.

\section{Spectrum}

The design ind construction of equipment to measure the low-frequency spectrum of the CBR has begun. Many theories predict that a spectral distortion in the cosmic microwave background is most readily observable in the Rayleigh-Jeans region. This is because it is much easier to modify the energy or create low-frequency (low-energy) photons than high-fiequency (high-energy) photons. 


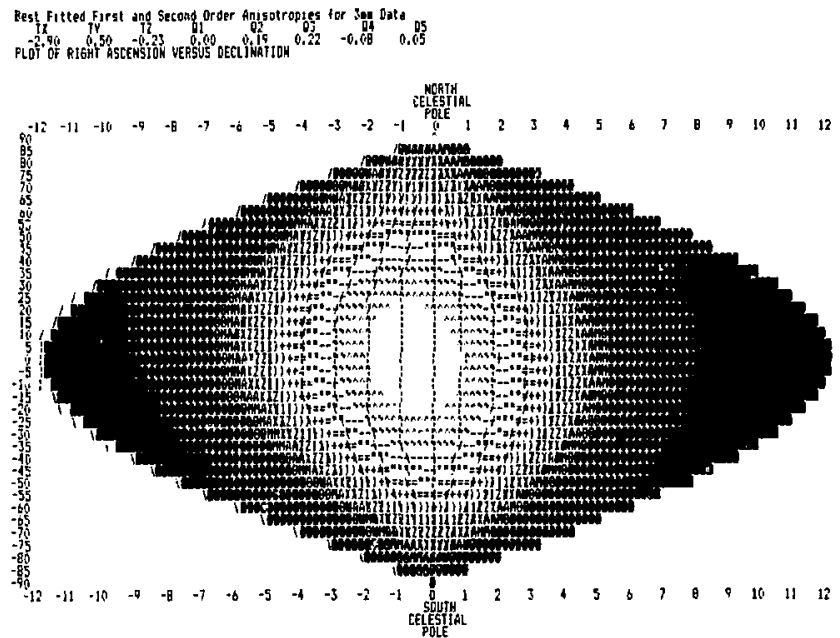

SAHFLE OF GRAY SCALE,

XBL 8210-2959

Fig. 21. Computer display of the intensity of the cosmic background radiation at a wavelength of $3 \mathrm{~mm}$, showing the anisotropy attributed to the motion of our galaxy.

During the summer of 1982 the group will make measurements of the low-frecuency spectrum of the CBR. The measurements will be made at five wavelengths $(12,6,3,0.9$, and $0.3 \mathrm{~cm})$ below the peak of the spectrum. The radiometers will use a single liquid-helium cold-load calibrator in order to reduce the systematic errors. The experiment will be done from the White Mountain High Altitude Research Station in California. It is a collaboration of groups led by Giorgio Sironi (Milano, Italy), Reno Mandolesi (Bologna, Italy), Bruce Partridge (Haverford College, Pennsylvania), and George Smoot (with the Astrophysics Group at LBL).

Previous measurements in this frequency region were made more than a decade ago by several groups using different techniques. This experiment will be using technology that was not available at that time. It suffers fewer systematic errors because it will use a single calibrator for all five radiometers, and the calibration will be referencent to the front end of each.

It is planned tc continue measurements of the low-frequency spectrum with development of recejvers that can be tuned over a wide frequency range. This should match and extend the previous and possibly repeated measurements at the five fixed wavelengths.

\section{Research Staff}

Giovanni De Amici, Jerry Epstein, Scott Friedman, John Gibson, Phil Lubis, Philip Melese-d'Hospital, George Smoot, Chris Witebsky, John Yamada.

Publications

Cosmic Quadrupole? R. Muller. Natuse 291, 609 (1981).

Large-Angular-Seale Arisotropy in the Cosmic Background Radiation. M. Gorenstein and G. Smoot. Ap. J. 244, 35 ( (1981).

Polarization of the Cosmic Background Radiation. P. Lubin and G. Smool. Ap. J. 245, I (1981).

\section{Ten-Meter Telescope}

The research effort of the group has become centered on the University of California's Ten-Meter Telescope (TMT) Project. A decision was made last year to build a full scale prototype, and the design and con- 
struction of this technical demonstration is now in progress. The goals are to design, build, and test all those commonents that are critical to the feasibility of the TMT. This involves most of the active control system for the primary mirror, and much of the passive support system for the mirror segments. Many of the problems anticipated in the optical fabriculion of the mirnor segments will also be addressed. Much of the design has been completed and fabrication is well underway.

Along with this effort, more general work to develop the design of the TMT itself is proceeding. Funding for this project comes entirely from the University of California.

The technical demonstration for the TMT is expected to be completed by early 1983 . This includes the testing of the components, the apparatus, and the control system. Pending additional funding, further detailing of the design of the TMT will begin and will involve the design of the telescope and additional design of the optical system.

A fund-raising campaign for the design and for the telescope project is underway. We will provide any technical assistance needed for this effort.

Assuming the success of the fund-raising campaign for the TMT, the detailed design of the entire observatory will begin. This includes the telescope, the building and dome, the optical fabrication facilities, the first round of scientific instrumentation, etc.

Actual construction of the observatory is expected to begin in 1984.

\section{Research Staff}

David Blakely, Michael Budiansky, Dave Cudaback, Terry Mast, Jerry Nelson, Barbara Schaefer, Marc Temple-Raston.

\section{Publications}

The Use of the Ten Meter Telescope at Infrared Wavelengths. $K$. Malthews. January 1981.

Estimation of Wind Loads and the Effects. J. Nelson. January 1981.

Mirror Segment Motions from Gravitational Deformations of the Cell. J. Nelson bnd T. Mas!. 1981

Geometrical Relations for an Altitude Azimuth Telescope. J. Nelson. January 1981.

Preliminary Design of the Tube for the TMT, J. Lubliner. January 1981.
Effexts of Altude on Performance and Health: Stress and $4 \mathrm{~km}$ High Telescopes. D. Curlaback. January 1981.

Setting Acceptable Limits for the Thermal Coefficient of Segments. J. Nelson. February 1981.

Soil Defornutions Due to Wind Action on the Building. $S$. Medwadowski. February 1981.

Project Summary for Executive Managemt i. Committec Meeting. Telesrope Design Team. February 11, 1981.

Formulae Relevant to the Optical Performance. S. Faber. April 1981.

The Contribution of Focus Errors to the Optical Error Budget. S. Faber. April 1981.

Estimated Image Diameters at 10 microns. S. Faber. April 1981.

Milestones for a Prototype Mirror. A Dubois. April 1981.

Conceptual Design of Structure of U.C. Team Meter Telescope: Final Keport, Phase II. S. Medwadouski. May 1981.

Funding Proposal for the Technical Demonstration and Preliminary Design of the UC TMT. Coordinator, A Dubois. June 1981.

The Optimum Match between Image Size and the Size of Detector Pixels: The Choice of Angular Scale. S. Faber. Jure 1981.

Matching Representative Instruments and Detoctors to the Ten Meter Telescope: Specific Examples. S. Faber. June 1981.

Science for the Ten Meter Telescope. S. Fober. June 1981.

Figure Confol for a Fully Segmented Primary Mirtor. T. Mast and J. Nelson. July 1981.

\section{Determination of the Atmospheric $\mathrm{CO}_{2}$ Budget}

The rise in the atmospheric $\mathrm{CO}_{2}$ concentration, due at least in part to the combustion of fossil fuels, is expected to have a profound impact on the climate everywhere on earth. The credibility of current predictions of the future rise of atmospheric $\mathrm{CO}_{2}$ is seriously impaired by the fact that the present $\mathrm{CO}_{2}$ budget does not seem to be in balance. Very precise messurements of changes in the global atmospheric $\mathrm{O}_{2} / \mathrm{N}_{2}$ ratio in the course of a few years could provide the ky to the solution of the "missing $\mathrm{CO}_{2}$ " problem.

This year an apparatus has been built that utilizes Raman scattering from a laser beam to make precise comparative measurements of the $\mathrm{O}_{2} / \mathrm{N}_{2}$ ratio in a sample and a standard. The scattering targets (air) are located inside the laser cavity. Interference filters match the spectral signatures of $\mathrm{O}_{2} / \mathrm{N}_{2}$. Coupled choppers rotating at about $50 \mathrm{~Hz}$ interchange the signal 
from sample and standard. Efforts are presently underway to obtain the required stability while just looking at the well-mixed air inside the scattering chamber.

\section{Research Staff}

Stu Bloom, Pieter Tans, Paul Weinstein.

Publications

${ }^{13} \mathrm{C} /{ }^{12} \mathrm{C}$ of Industrial $\mathrm{CO}_{2}$. P. Tans. In Carbon Cycle Modelling, pp. 127-130. Ed, R Bolin (SCOPE Report 16). John Wiley, 1981.

A Compilation of Bomb ${ }^{14} \mathrm{C}$ Data for Use in Global Carbon Model Calculations. P. Tans. In Carbon Cycle Modelling, pp. 131-158. Ed. R. Bolin (SCOPE Report 16), John Wiley, 1981.

\section{Very Small Cyclotron}

A small low energy cyclotron is being developed for the purpose of ultrasensitive detection of long lived radioisotopes such as ${ }^{10} \mathrm{Be}\left(\mathrm{t}_{1 / 2}=1.6 \times 10^{6}\right.$ years $),{ }^{26} \mathrm{Al}$ $\left(t_{1 / 2}=0.72 \times 10^{6}\right.$ years $),{ }^{36} \mathrm{Cl}\left(t_{1 / 2}=0.30 \times 10^{6}\right.$ years $)$, and ${ }^{129} \mathrm{I}\left(t_{1 / 2}=16 \times 10^{6}\right.$ years $)$. Mass spectrometry using cyclotrons and tandem Van de Graaf accelerators has proven to be a far more sensitive means of detection of many long lived radioisotopes than other methods. Minimum sample sizes are in most cases more than two or three orders of magnitude smaller. Measurements of
${ }^{10} \mathrm{Be},{ }^{36} \mathrm{Cl}$, and ${ }^{129} \mathrm{I}$ in meteorites help determine the cosmic ray bombardment history as well as the terrestrial age of the meteorite. Measurements of ${ }^{10} \mathrm{Be}$ and possibly other radioisotopes in the atmosphere can indicate the movement of stratospheric air, where they are most abundant, into the troposphere. The low energy cyclotron will use the great selectivity and suppression of ions on nonresonant $\mathrm{g} / \mathrm{m}$ of the cyclotron to perform particle jdentification. Unlike high energy accelerators, this low energy machine does not require massive radiation shielding, high magnetic fields, or a large magnet; and it will be dedicated solely to radioisotope measurements.

Development is continuing of ion sources appropriate for use in the 88-Inch Cyclotron external ion source that have fast sample changing capability and can ionize solid samples, which is essential for this method of radioisotope dating. The 88-Inch Cyclotron has been used to produce the rare radioisotope ${ }^{10} \mathrm{Be}$ wtich will be used to calibrate the amount of ${ }^{10} \mathrm{Be}$ in sar: jles to be measured.

Research Staff

Branch Archer, Peter Friedman, Don Morris, Richard Muller, Jim Welch.

Publications

Mass Spectrometry with a Very Smalt Cyciotron. R. Multer, et al. LBL 12797 .

\section{Detector and Other Research and Development}

\section{R \& D Using TPC Concept}

The technique of drifting ionization over long distances used in the PEP-4 TPC finds natural extensions in attempting several new experiments. A group of physicists who collaborate on the PEP-4 TPC is also carrying on an active program of research and development extending TPC concepts to new regimes.

\section{High Background Environments: Gated Grid}

Up to now the TPC techrique has been applicable on'v in low rate and low background environments. The long drift associated with the TPC necessarily impiies that a long time is needed to collect and clear the positive ions generated in the detecting elements. The posi- tive ions distort the TPC electric drift field and create track distortions. This limitation in be avoided by using switching grids to close the electric field lines that normally connect the detecting elements to the long drift region.

The group has operated a small prototype in a fully gated mode. They have demonstrated that a tetrode geometry is needed to perform the switching without affecting the sensitive electronics coupled to the detecting elements. They have also confirmed calculations on the transparency and closure of the gate, determined the tolerances needed to build gated grids, and developed a pulser capable of performing the switching functions adequately. Future work associated with the gated grid will involve building a full-size grid for the PEP-4 TPC. 
Muon Neutrino Mass: Tracking Imaging Cerenkov Counter

The present upper limit on the muon neutrino mass, namely $0.5 t \mathrm{MeV}$, could allow this "massless" particle to be as heavy as the electron. It appears possjble, however, to improve this mass limit significantly by a new approach: the use of a segmented differential cerenkov counter with internal tracking for a precise measurement of the velocities in pion decay in flight: $\pi \rightarrow \mu \nu$.

The experiment requires a clean pion beam of a few $\mathrm{GeV} / \mathrm{c}$ such as may be provided by the Brookhaven AGS. The differential counter with internal tracking is needed to prevent multiple scattering and energy loss from destroying the otherwise superb velocity resolution of Cerenkov counters. The present research and development effort is aimed at building one segment of the approximately fifty segments needed in the final experiment. The present plan is to operate a modest hydrugen TPC with approximately $10-\mathrm{cm}$ drift inside a $1-\mathrm{m}$ long counter. The TPC measures the particle trajectory without obscuring the Cerenkov light cone. The position of the Cerenkov photons is measured in a position sensitive image intensifier. In this arrangement each photon gives an independent measurement of the particls velocity. The eventual experiment depends crucially on the resolution achieved in the prototype counter, and the research and development effort is designed to demonstrate that the expected resolution is in fact achievable.

\section{New Proton Decay Detector: Higher Density TPC}

The groul is exploring the possibility of using an yltra-high pressure TPC (500 atm) to study nucleon decay. At such high pressures the density of argon is approximately equal to the density of water, and the device becomes not only a tracking detector but also a detector capable of measuring total energy. A 10-kton detector would be approximately $22 \mathrm{~m}$ on a side and would be operated in the ocean at approximately 5-km depth. All particles from proton decays would be contained in the detector. Multiple measurements of the ionization $\mathrm{dE} / \mathrm{dx}$ along the tracks, together with the integral of $\mathrm{dE} / \mathrm{dx}$ along the tracks (total energy) would completely identify the types of particles present in the decays.

The group is investigating the operation of proportional cells in a 500 -atm environment, as well as the difficulties in drifting electróns at such densities. They use a counter of 8 -in. diameter and $72 \mathrm{in.}$ long to test electrostatic stabilities of various cell designs, purity requirements and methods of recirculating and cleaning the gas.

A proposal actually to build a complete proton decay detector would represent a major commitment for the Laboratory and will await completion of the research program.

\section{Research Staff}

Peter Nemethy, Piermaria J. Oddone, William Wenzel.

\section{Electron-Photon Calorimetry}

Two new techniques of wire calorimeter design are currently under investigation for possible use with the Mark II Detector at the SLC. These involve use of the streamer mode or the saturated avalanche mode for the operating region of the sense wires. These modes each have two advantages: (1) much higher gains, which eliminates the need for preamplifirss, and (2) some pulse height saturation, which suppresses the delta rays that degrade the calorimeter energy resolution. In addjtion, investigations are being conducted of the newly discovered electrodeless drift chamber principle, by means of which spontaneous charging of the inside walls of the chamber causes a uniform drift field to be generated. The self-compensating nature of this device has some very attractive features for its potential use in drift calorimeter design, because of the lack of sensitivity to geometry, or possibly even the insensitivity to placement in magnetic fields.

\section{Research Staff}

John Kadyk

\section{High Resolution Vertex Detector Development}

Work continued on device studies and prototyping for high-resolution, sampling, solid-state detectors. (See Fig. 22.) Part of the work has been based on hybrid CCD technology, in particular, in copperation with Rockwell International on a $32 \times 32$ element CCD. Work is continuing on low-noise electronics, including clock drivers; double-correlated sample and hold techniques; and low-noise, high-speed amplifiers. In parallel to this effort design work has begun on a thick, ultra-high-purity silicon detector suitable for connection to the Rockwell CCD Multiplexer. Hybrid device studies include noise and efficiency measurements, radiation damage effects and beam studies.

In addition consideration has been given to the possible use of optical CCDs for a high-resolution vertex detector for the SLC. (See Fig. 23.) Studies have already been completed using a Fairchild optical CCD, and they have shown the viability of such a detector. A collaborative effort is now under way with SLAC in order to do beam studies with a CCD telescope consist- 


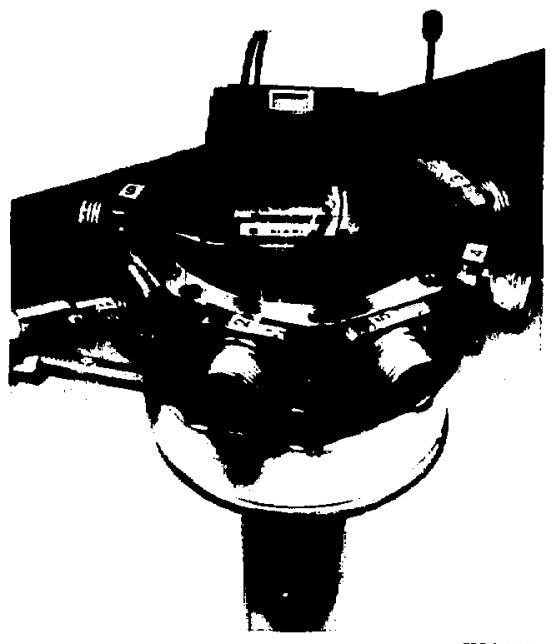

Fig. 22. Experimental charge-coupled device (CCD) detector mounted on a test stand. The actual CCD unit can be seen inside the lighter toned rectangular frame at the top of the setup. (CBB 810-9604)

ing of 4-5 CCDs. The beam tests are scheduled for the fall of 1982. All of the low noise electronics studies will be directly applicable to this effort.

\section{Research Staff}
A. Bross.

\section{Publications}

High Resolution, Pesition Sensitive, Solid State Detectors Utilizing the CCD Concept. A. Bross. Paper presented at Silicon Detector Workshop, Fermilab, Oct. I5-I6, I8I.

\section{Monopole Search}

A proposal to look for magnet monopoles in flight is being considered by the Physics Division. Several square meters of superconducting shield would be
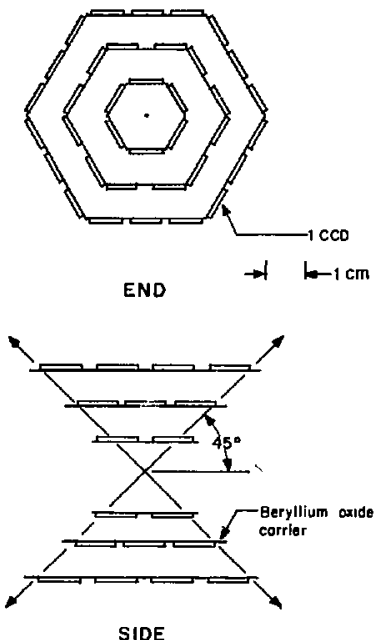

TOTAL NO. OF CCO'S = $2 \times 6+3 \times 12+4 \times 18=120$

X8L 821-7567

Fig. 23. Conceptual design of a possible vertex detector for an $\mathrm{e}^{+} \mathrm{e}^{-}$collider using 120 CCDs.

mapped periodically, during a year or so. The experiment could detect monopoles predicted by the grand unification theories if they are present in the cosmic rays at the level of $1 / \mathrm{m}^{2}$ year. No other experiment could have detected them at this low fiux level.

\section{Research Staff}

P. Eberhard.

Publicitions

Possibjlity of New Monopole Search. P.H. Eberhard. Physics Note No. 912 (1981).

A Detector for Magnetic Monopoles in Flight. P.H. Eberhard. Physics Note No. 925 (1981). 


\section{NMR Imaging}

A new technique for NMR imaging was suggested at the end of 1981 . The basic principle should be tested during calendar 1982 using existing surplus normal coils. If the tests confirm that the spatial resolution is better and/or that the spectrum of time constants is easier to investigate than the present techniques, a proposal for a large scale program of NMR imaging of the human body will be submitted to NIH.

\section{Research Staff}

P. Eberhard, R. Ross.

Publications

Threc Dimensional NMR Imaging. P.H. Eberhard. Physies Note No. $923(1981)$

Conments About Tomography. P.H. Eberhard. Physies Note No. 924 (1981).

\section{Gas-Filled Detectors}

Development work was done on various forms of gas-filled multi-electrode detectors. This included studies of the properties of various gas mixtures to provide (a) higher electron drift velocity, (b) higher avalanche gain by use of organic low ionization potential additives, (c) limiicd streamer discharges using hydrocarbon and carbon dioxide additives.

a) Work on gas-filled detectors capable of recording large multiplicity events by creating glow discharge memories at the locations of their tracks was continued. Various configurations of wire planes, gas fillings and readout electronics were investigated. A final configuration has been selected and an experimentally usable prototype is under construction.

b) Developments and measurements were done on gas-filled linear chambers with delay line readout for various applications including high-precision, small-angle scattering of $x$ rays from metallic alloys and powder samples. This work, done in cooperation with the metallurgy group of $D$. de Fontaine (MMRD), allowed us to investigate the properties of fast printed circuit delay lines capable of high data rates and position accuracy in the $100-\mu \mathrm{m}$ region. Simple high-speed electronics developed for this application using I.C. amplifiers and comparators enabled both of our objectives to be realized. c) Applications of gas-filled detectors in biomedical and biophysical problems have been continued. Lead glass converters for high efficiency detection of $\gamma$ rays in the energy range $200 \mathrm{keV}-20 \mathrm{MeV}$ have been made with high density $(p=6.3$ $\mathrm{g} / \mathrm{cm}^{3}$ ) out of fused lead glass tubing. The fused lead glass matrices are made surface conducting by high temperature reduction in a hydrogen furnace. Interaction electrons produced by incident gamma rays are drifted out of the glass matrix and into the wire plane region of MWPC, thus providing position resolution. Applications of these to medical positron imaging cameras and to position imaging of electron distributions within crystals are proposed. Work is also continued on computer methods to solve for the electron density distribution within three-dimensional objects by imaging with annihilation gammas from positron capture or by single gamma-emitting radioisotope distributions within the object. These computer methods are applicable to MWPC detectors covering finite angles or in completely different fields such is electron microscopy, or for study of reactor cores, using MWPC with ${ }^{10} B$ plates for neutron detectors.

\section{Research Staff}

Alberto del Guerra, E. Grove, Terrance A. Mulera, Victor Perez-Mendez, G. Schwarz, Bradley Sleaford, Pamela E. Wiedenbeck.

Publications

Computer Graphic Display of Cardiac C.T. Scans. Paimer, Yen, Kuo, Feinberg. Wiedenbeck, Perez-Mendez, Skioldebrand, Carlsson. Cardiovascular Interventional Radiology 5 (1982).

Multiplicity Detector Using a Glow Discharge Memory. Mulera, Elola, Perez-Mendez, Wiedenbeck. IEEE Trans. Nuc. Sci. NS-29 (1982), 425.

Small Angle X-Ray Scattering System with Linear Position Sensıtive Delector, Forouhi, Perez-Mendez, Sleaford, De Fontaine, Fodor. LBL-12587. IEEE Trans. Nuc. Sci., NS-29 (1982), 257.

Contribution of Time-of-Flight Information to Limited Aingle Positron Tomography. MacDonald, Perez-Mendez. Tam IEEE Trans. Nuc. Sci. NS-29 (1982), 510.

Otservation of Large Saturated Pulses in Wire Chambers Filled with Argon-Carbon Dioxide Mixtures. T. Mulera and V. Perez-Mendez, LBL-14003 (March 1982). To be published in N.I.M. 
Medical Positron Imaging with a Dense Drift Space Multi-wire Pro portional Chamber. A Del Guerra, C. B. Lim, G. K Lum, D. Orendahl and V. Perez-Mendez, LBL-14043 (March 1982). To be published IEEE Trans, Nuc. Sci.
Lnrge Solid State Detectors, V. Perez-Mendez and P. Wiedenbeck, LALPUB-5069.

\section{Publications and Reports of Other Research}

\section{Research at LAMPF}

Pion Double Charge Exchange on "He and Meson Exchange Currents. Davis, Carroll, Fawcet, Kallne, Minehart, Stetz, Segle, PerezMendez, MoClelland, Whitney. Phys. Rev. Lett, 47 (1981) 782-785.

Pion Double Charge Exchange on ${ }^{3} \mathrm{He}$ and ${ }^{4} \mathrm{He}$. Stetz, Swanson, Davis, Kallne, Minehard, Fawcelt, Perez-Mendez, Sagle, Carroll, Meclelland, Whitney. Oregon State University preprint (Deomber 1981). To be published Nucl. Phys.

Muon Decay Neutrinos and Limits on Neutrino Oscillations. Gauge Theories, Massive Neutrinos and Proton Decay. P. Nemethy. Proceedings of Orbis Scientiae 1981; edited by A Perlmutter. pp. 263-270. PJenum Press (1981).

Multiplicative Versus Additive Muon Conservation. P. Nemethy and v.W. Hughes. Comments on Nucl. and Part. Phys. 10, 147 (1981).

\section{CELLO Detector at PETRA}

CELLO - A New Detector at PETRA H.-J. Behrend, J. Field, V. Schroder, H. Sindt. W.-D. Apel, J. Bodenkamp, D. Chrobaczek, J. Engler, D. C. Fries, G. Fluzge, H. Muller, H. Randoll, G. Schmid, H. Schneider, W. de Boer. G.Buschhorn, G. Grindhamuner, P. Grosse-Wiesmann, B. Gunderson, C. Kiesling, R. Kotthaus, H. Lierl, D. Luers, T, Meyer, L. Moss, H. Oberlack, P. Schacht, M.-J. Schachter, A Snyder, H. Steiner, G. Carnesecchi, A Cordier, M. Davier, D. Fournier, J. F. Grivaz, J. Haissinski, V. Journe, F. Lalache, F. Le Diberder, J.-J. Veillet, A Weitsch, R. Goorge, M. Goldberg, B. Grossetete. F. Kapusta, F, Kovaes, G. London, L Poggioli, M. Riyoal, R. Aleksan, J. Bouchez, G. Cozzika, Y. Ducros, A Gaidot, J. Pamela. J. P. Pansart, and F. Pietre. Physica Scripla 23, 610 (1981).

Measurement of $e^{+} e^{-\rightarrow} e^{+} e^{-}$and $e^{+} e^{-\rightarrow \gamma r}$ at Energies up to 36.7 GeV. H.-J. Behrend, J. Field, V. Schroder, H. Sindt, W.-D. Apel, J. Bodenkamp, D. Chrobaczek, J. Engler, D. C. Fries, G. FlugBe, H. Muller. H. Randall, G. Schmidt, H. Schneider, W. de Boer, G. Buschhom, G. Grindhamurer. P. Grosse-Wiesmann, B. Gunderson, C. Kiesling, R. Kotthaus, H. Lierl, D. Luers, T. Meyer, L. Moss. H. Oberlack, P. Schachh M.-J. Schachter, A. Snyder, H. Stciner. G. Carnesecchi, A. Cordier, M. Davier, D. Fournier. J. F. Grivaz, J. Haissinski, y Journe, F, Lalache, F, Le Diberder, J.-J. Veillet, A Weitsch. R. Grorge. M. Goldberg, B. Grossetete, F. Kapusta, F. Kovacs, G. London, L Poggioli, M. Rivoal, R. Aleksan, J. Bouchez, G. Cozzika, Y. Ducros, A Gaidot, J. Pamela, J. P. Pansart, and F.
Pierre. Physics Letters, 103B, 148 (198I)

Measurement of the Reaction $e^{+} e^{-} \rightarrow \pi^{+} \pi^{-}$at PETRA, CELLO, H.-J. Behrend, J. Field, V. Schroder, H. Sindt, W.-D. Apel, J. Bodenkamp. D. Chrobaczek, J. Engler, D. C. Fries, G. Flugge, H. Muller, H. Rendoll, G. Schnidh, H. Schneider, W. de Boer, G.Buschhorn, G. Grindharnmer, P. Grosse-Wiesmann, B. Gunderson, C. Kiesling, R. Kotthaus, H. Litrl, D. Luers, T. Meyer, L. Moss, H. Oberlack, P. Schacht, M.-J. Schechter, A Snyder, H. Steiner, G. Carneseochi, A. Cordier, M. Davier, D. Fournier, J. F. Grivaz, J. Haissinski, V. Journe, F. Lalache, F. Le Diberder, J.-J. Veillet, A Weitsch, R. Goorgh M. Goldberg, B. Grossetete, F. Kapusta, F. Kovacs, G. Lor don, L. Poggioli, M. Rivoal, R. Aleksan, J. Bouchez, G. Cozzika, Y. Ducros, A Gaidot, J. Pamela, J. P. Pansart, and F. Pierre. Contributed paper to International Conference on High Energy Physics, Lisbon, July 1981 .

Search for Scalar Leptons at PEJRA, CELLO. H.-J. Behrend, J. Field, V. Schroder, H. Sindt, W.-D. Apel, J. Bodenkamp. D. Chro baczek, J. Engler, D. C. Fries, G. Flugge, H. Muller, H. Randall, G. Schmidt, H. Schneider, W. de Boer, G.Buschhorn, G. Grindhammer, P. Grosse-Wiesmann, B. Gunderson, C Kiesling, R. Katthaus, H. Lie?l, D. Luers, T. Meyer, L Moss, H. Oberlack, P. Schacht, M.-J. Schachter, 凡 Snyder, H. Steiner, G. Carnesecchi, A Cordier, M. Davier, D. Fournier, J. F. Grivaz, J. Haissinski, V. Journe, F. Lalache, F. Le Diberder, J.-J. Veillet. A Weitsch, R. George M. Goldberg. B. Grossetete, F. Kapusta, F. Kovacs, G. London, L. Poggioli, M. Rivaal, R Aleksan, J. Bouchez, G. Cozzika, Y. Ductos, A Gaidot, J. Pamela, J. P. Pansart, and F. Pierre. Contributed paper to International Conference on High Energy Physics, Lisbon, July 1981.

Searcb for Narrow Quarkonium States and Pair Production of New Heavy Quarks at C.M. Energies of $25 \mathrm{GeV}$ and from 33.0 to 36.7 GeV. H.-J. Behrend, J. Field, V. Schroder, H. Sind, W.-D. Apel, J. Bodenkamp, D. Chrobaczek, J. Engler, D. C. Fries, G. Flugge, H. Muller, H. Randoll, G. Schmidt, H. Schneider, W. de Boer, G. Buschhorn, G. Grindhammer, P. Grosse-Wiesmann, B. Gunderson. C. Kiesling, R. Kotthrus, H. Lierl, D. Luers, T. Meyer, L Moss. H. Oberlack. P. Schachi, M.-J. Schachter, A Snyder, H. Steiner, G. Carnesecchi, A Cordier, M. Davier. D. Fournier, J. F. Grivaz, J. Haissinskj, V. Journe, F. Lolache, F. Le Diberder, J.-J. Veillet, A Weitsch, R. George M. Goldberg, B. Grossetete, F. Kapusta, F. Kovacs, G. London, L Poggioli, M. Rivoal, R. Aleksan, J. Bouchez, G. Cozzika, Y. Ducros, A Gaidot, J. Pamela, J. P. Pansart, and F. Fierre. Contributed paper to International Conference on High Energy Physics, Lisbon, July 1981. 
Comparison of the Neutral and Charged Components of Hidronic Jets in $\mathrm{e}^{+} \mathrm{e}^{-}$Annihilation at Energies up $1036.7 \mathrm{CeV}$. H.-J, Eehrend, J, Field, V. Schroder, H. Sindi, W.-D. Apel, J. Bodenkamp, D. Chrobasczek, J. Engler, D. C. Fries, G. Flugge, H. Muller, H. Randoll, G. Schmidt, H. Schneider, W. de Bocr, G.Buschhorn, G. Grindhammer, P. Grosse-Wiesmann, B. Gunderson, C. Kiealing, R. Kothaus, H. Lierl. D. Luers, T. Meyer, L Moss, H. Oberlack, P. Schacht, M.-J. Schachter, A Snyder, H. Steiner, G. Carnesecchi, A. Cordier, M. Davier, D. Fournier, J. F. Grivaz, J. Haissinski, V. Journe, F. Lalache, F. Le Diberder, J.-J. Veillet, A Weitseh, R. Goorge, M. Goldberg, B. Grasseteie, F. Kapusta, F. Kovacs, G. Landon, L Poggioli, M. Rivoal, R. Aleksan, J. Bouchez, G. Cozzika, Y. Ducros, A Gaidot, J. Pamela, J. P. Pansart, and F. Pierre. Contributed paper to International Conference on High Energy Physics, Lisbon, July [98I.

Measurement of the Reactions $r y \rightarrow \pi^{+} \pi^{-}+\pi^{-}, \mathrm{m}^{-f_{0} \rightarrow x^{+}} \pi^{-}$with the CELLO Detector at PETRA, CELLO. H.-J. Behrend, J. Field, V. Schroder, H. Sindt, W.-D. Apel, J. Bodenkamp, D. Chrobaczek, J. Engler, D. C. Fríes, G. Flugge, H. Muller, H. Kandoll, G. Schmidt, H. Schneider, W. de Boer, G.Buschhorn, G. Grindhamuer, P. Grosse-Wiesmann, B. Gunderson, C. Kiesling, R. Kotthaus, H. Lierl, D. Luers, T. Meyer, L Moss, H. Oberlack, P. Schacht, M.-J. Schachter, A Snyder, H. Steiner, G. Carnesecchi, A Cordjer, M. Davier, D. Fournier, J. F. Grivaz, J. Haissinsk, V. Journe, F. Lalache, F. Le Diberder, J.-J. Veillel, A Weitsch, R. George, M. Goldberg, B. Grossetete, F. Kapusta, F. Kovacs, G. London, L. Poggioli, M. Rivoal, R. Aleksan, J. Bouchez, G. Cozzika, Y. Ducros, A Gaidot, J. Pamela, J. P. Pansart, and F. Pierre Contributed paper to International Conference on High Energy Physics, Lisbon, July 1981.

\section{Research at Fermilab}

\section{Neutrino and Anti-Neutrino Reactions}

Dilepton Production by Neutrinos in the Fermilab 15-Foot Bubble Chamber. H.C. Ballagh, et al. Phys. Rev. D 24, 7 (July 1981).

Evidence of Hard-Gluon Bremsstrahlung in a Deep-Inelastic Neutrino Seattering Experiment. H.C. Ballagh, et at. Phys. Rev. Letters, 47, 556 (Augus! 1981).

\section{Elastic Scattering}

A High Statistics Study of $\pi^{4} p, \pi p$, and pp Elastic Seattering at 200 GeV/c. A Schiz, J.N. Merk, et al. Phys. Rev. D 24 (1981) 26. The Real Part of the Forward Elastic Nuclear Amplitude for pp, $\bar{p} p, \pi^{+} p$, $\pi^{-p}, K^{+} p$, and $K-p$ Seattering Between 70 and $200 \mathrm{GeV} / \mathrm{c}$, L. A. Fajardo, R. Majka, J. N. Marx, P. Nemethy, L. Rosselet, J. Sandweiss, A. J. Slaughter, C. Ankenbrandi, M. Arac, R. Brown, S. EckJund, P. J. Gollon, J. Lach, J. Meclachlan, A. Roberts, G. Shen. Phys. Rev D 24 (1981) 46.

\section{Kaonic-Atom X Rays}

Ksonic Alass by Critical Absorption of Kaonic-Alom $X$ Rays. C.K.
Lum, C. E Wiegand, E. G. Kessler, Jr., R. D. Deslattes, L. Jacobs, W. Schwitz, R. Sekj. Phys. Rev. D 23, 2522 (1981).

\section{Cryogenics and Superconducting Magnets}

Cryogenic Tests of Glass-Epoxy Based Electrical Insulation. J.D. Taylor, P. S. Martin, M. Pripstein, M. A. Green In Proceedings of the 8th International Cryogenic Materials Conference, San Diego, CA (August 1981) and to be published in Advance in Cryogenic Engineering, Vol, 28. LBL- 12782.

Gas Cooled Electrical Leads for Use on Forced Cooled Superconducting Magnets. R. G. Smits, P. L Andrews, W. A. Bums, C T. Day, J. W. Gary, G. H. Gibsan, M. A. Green, M. Pripstein, R. R. Rass, J. D. Talor. Presented at the Cryogenic Engineering Conference, San Diego, CA (Augusi 1981) and to be published in Advances in Cryogenic Engineering, Vol. 27. LBL-12783.

The Measurement and Theoretical Calculation of Quench Velocity within Large Fully Epoxy Impregnated Coils. P. Eberhard, R. R. Ross, J. D. Taylor. Th International Conference on Magnet Technology IEEE Transactions on Magneties, Vol. MAG 17, 51803 (March 1981). LBL-12327.

Enthalpy Changes in Superconducting Wires. P.H. Eberhard. Physies Note No. 914 (1981).

Deteeting a Change in Quantity of Copper in a Rectangular Superconductor with an Eddy Current Change Detector. G.H. Gibson and R.G. Smits. Physies Note No. 918 (1981).

\section{Miscellaneous}

Antiproton-Nucleon Experiments. R.D. Tripp. In Proceedings of the Workshop on Nuclear and Particle Physics al Energies up to $31 \mathrm{GcV}$ : New and Future Aspects, Los Alamos, NM (January 1981).

Upper Limil on the Branching Fraction $x(3554) \rightarrow \sqrt{\pi}+\pi^{+}{ }^{0}$. R. Barate, P. Bareyre, P. Bonams, P. Borgeaud, M. David, F. X. Gentit, G. Laurens, Y. Lemoigne, G. Villet, S. Zaninoitti, P. Astbury, A Duane, G. J. King, B. C. Nadi, R. Namjoshi, D. M. Websdale, J. Wiejak, J. G. MeEwen, B. Pitrzyk, R. Tripp, B. B. Brabson, R. Crittenden, R. Heinz, J. Krider, T. Marshall. Phys. Rev D 24, 2994 (1981).

Reactions $K^{-} \mathbf{P} \rightarrow \Sigma^{-}+$and $K \longrightarrow p \rightarrow \Sigma^{+}{ }^{-}$in the Momentum Range from 220 to $470 \mathrm{MeV} / \mathrm{c}$. R.O. Bangerter, M. Alston-Garnjost, A Barbaro-Galtieri, T. S. Mast, F. T. Solmitz, and R. D. Tripp. Phys. Rev, D 23, 1484 (April 1981). 


\section{Computation and Communication}

To provide computation facilities for the high energy physics program, the Division has installed a computer system at LBL and has implemented a highspeed microwave system between LBL and SLAC.

The computer system is used for off-line analysis of data for the TPC and from other experiments. In addition the facilities are used for program development, general computation and for the development and testing of detectors. The system consists of a VAX $11 / 780$ processor, three high density tape drives and three large disks. Interactive computing is provided through the Computer Center's Develcon switch. Computer network hardware and Decnet software are used to link DEC computers at LBL, at PEP, and at a number of universities where groups are collaborating in PEP experiments.

The microwave system utilizes transmitters and receivers at LBL and SLAC to provide high-speed computer-to- computer networking, terminal-to-computer communication, and voice lines. There are at present 8 channels linking terminals at LBL to the SLAC central computing facility, 15 channels connecting LBL terminals to online computers at PEP, and 5 lines linking terminals at PEP to LBL computers. There is also a direct telephone link connected to the LBL Centrex service. A microwave Decnet link permits transfer of up to 230,400 bits per second between the TPC online computer at PEP and the Physics VAX at LBL. The system has been designed with extensive growth capability, and it is expected that more links will be added in the near future.

The microwave link was originally established to provide a TV conferencing facility. It is still used for that purpose and has been enlarged to include two rooms at SLAC (one in IR-2 at PEP via an optical fiber cable) and three rooms at LBL.

\section{Research Staf}

Christopher Day, Stewart C. Loken, Edgar T. Whipple.

\section{Engineering, Evaluation, and Support Operations}

The development engineering of instruments and instrumentation systems which have specific as well as broad and general applications is a continuing task. Other tasks include the research and development of particle detectors and the evaluation of commercially available devices and instruments. Operating manuals are supplied, and maintenance and support of instruments in the Counting Pool as well as previously designed systems used in HEP experiments are provided. In addition, inventory records of all equipment are maintained, new equipment is procured, and consulting service on all aspects of instruments and detectors used in research programs is provided.

\section{Large Scale Digitizer System}

The Large Scale Digitizer (LSD) System has been a continuing activity of the group since 1976. The LSD is an economical modular system for simultaneous digitization of analog data from hundreds of signal channels. Oider LiD systems are reconfigured and reused to meet new experimental requirements as they arise.

\section{Multiwire Proportional Chambers}

Several multiwire proportiunal chambers (MWPC) hove been constructed and installed this past year. A 16-channel preamplifier was developed for one of the MWPC systems to prolong the life of the detector by allowing lower voltages to be used on the chambers. Ongoing technical support for the MWPC systems in the field is provided.

\section{Hybrid CCD Solid State Detector}

The nirst phase for the development of the CCD driver electronics and readout-buffer memory system has been completed. The hybrid CCD solid state detector will be used as a high-resolution, position-sensitive target for neutral-beam, high-energy experiments. The second phase of electronic development has already begun.

\section{Microcomputer Systems}

Three microcomputers (two LSl-11 and one PET) continue to be used and developed to aid in test and checkout of various electronics subsystems and systems. Software is being developed to go along with hardware improvements.

\section{PEP-4 TPC System}

Specifications and partial design for a high-voltage interlock and protection system for TPC have been 
made. Installation and checkout of the Hex Calorimeter electronics as well as other miscellaneous jobs have taken place this last year.

\section{Technical Support and Pool Operations}

Services are provided in connection with all electronic instruments used in Physics Division programs, both on site and off site, on a continuing basis. Repair and maintenance of electronic equipment, maintaining inventory records, arranging temporary loans among research groups, procurement of new equipment, and providing operating manuals and consulting on all aspects of instruments and detectors are the types of services rendered. The base of the equipment pool continues to expand by coordinating it with those serving other LBL divisions. The operation of this combined multi-division equipment pool provides better utilization of equipment than the previous separate pools.

\section{Research Staff}

G. A. Constantian, E. J. Lampo, A E. Larsh, K. L.
Lee, B. Leskovar, C. C. Lo, M. Nakamura, C. Nunnally, S. R Olson.

\section{Publications}

A Time Projection Chamber Digitizer Test System Using a Microcomputer. C. Nunnally. IEEE Transactions on Nuclear Science (19811), Vol. NS-28, No. 2, p. 394.

A Triangular Tube Proportional Wire Chamber System D. H. Badtke, J. A Bakken, B. A. Barnett. B. I Bismenfeld. C. Y. Chien. L Madansky, J. A J. Matthews. A Persner, W. J. Spangler. K L. Lec, M. Nakamura and S. R. Olson. NIM (1981) 18B, 497-506.

Evaluation of the New Generation RCA8854 Photomultiplier. C. C. Lo and Branko Leskovar. LBL-13489, Presented at IEEE Nuclear Science Symposium San Francisco, October 1981.

Afterpulse Time Spectrum Measurement of RCA88850 Photomultiplier. C C. Lo and Branko Leskovar. LBL I 3619, Dec. 1981. 
THEORETICAL PHYSICS 


\section{THEORETICAL PHYSICS STAFF}

Abarbancl. Henry

Bardakei, Korkut

Cahn, Robert iv.

Caldi, Daniel G.

Chanowilz, Michael S.

Chew, Gooffirey F.

Gaillard, Mary $\mathrm{K}$

Halpern, Martin B.

Han, Chang G.

Hinchlifte, lan

Hung. Pham

Jackson, J. Dayid

Juod, David L
Lepore, Joseph V.

Mandelstam, Stanley

Neunann, Luanne K

Overacker, Susan J.

Riddell, Robert J.

Sheimnn, Jonathan L

Shizuye, Ken-ichi

Snydermar, Neal

Stapp, Henry $P$.

Stcrling. Thomas

Sublett, Betiy J.

Suzuki, Mehiko

Zumino, Bruno

\section{GRADUATE STUDENT RESEARCH ASSISTANTS}

Axelrod, Alan

Batrouni, Ghassan

De Forcrand, Philippe

Elser, Veit
Knng, Young

Levinson, Mark A.

Sharpe, Stephen R.

Smith, Adlai 


\section{THEORETICAL PHYSICS RESEARCH}

\section{Particle Physics}

The group in theoretical high-energy physics pursues a broad mix of research. Much of this work, e.g., the efforts in perturbative QCD, meson and glueball spectroscopy, and weak interactions, closely relates to the experimental program. Other research topics, such as work in quantum gravity, supergravity and supersymmetry, and nonperturbative QCD, involve fundamental issues that are not as closely related to the experimental program. Some of the particular research topics are described in more detail below. In addition, a substantial fraction of the theory group effort is devoted to supporting the experimental program, by formal and informal means such as workshops, seminars, and discussions on the planning and interpretation of experimental research.

\section{Supersymmetry and Grand Unification}

Supersummetry offers a hope of understanding the disparate hierarchy of scales in grand unified theories. This hope is as yet unrealized because of the extreme difficulty of constructing a realistic model based on broken supersymmetry. Gaillard, and independently, Cahn, Hall, and Hinchliffe have attempted to construct models. Chanowitz and Sharpe have studied the binding of gluinos in color-singlet hadrons. In related work, Zumino, with Wess, has studied conditions required for the spontaneous breaking of supersymmetry.

\section{Perturbative QCD}

With Furman, Haber, and Sheiman, Hinchliffe has evaluated high order corrections in a variety of processes, finding distressingly large results that cast doubt on the applicability of perturbation theory at presently available energies. He and Sheiman have considered predictions for quarkonium decays that are widely presumed to be independent of the structure of the potential (e.g., ratios of decays) but that they find have unmanageably large corrections, unless the bound state radius is much less than $\Gamma_{Q} \overrightarrow{C D}$. Sharpe has computed the one loop corrections to $\mathrm{e}^{+} \mathrm{e}^{-} \rightarrow \overline{\mathrm{q}} \mathrm{qg}$ and had identified the shape of the three jet angular distribution as a reliable test of QCD at PEP and Z-factory energies. Axelrod las used the operator product expansion to analyze photon-photon scattering for both photons offshell, which should give a useful QCD test at $\mathbf{Z}$ factory energies.

\section{Nonperturbative Field Theory}

Halpern, independently and with Schwartz, has developeó systematic classical methods for extracting the quantum physics of any Hamiltonian in the large $\mathbf{N}$ limit. Bardakci has derived the large $\mathbf{N}$ equations of QCD and used them to construct a model of the vacuum energy, of which he then studied the phase structure. Snyderman has studied static baryons in a generalized version of Polyakov's compact QED model in 2+1 dimensions. Kowall and Greensite have used computer methods to determine what fie!d configurations are responsible for confinement in lattice QCD. Though his immediate interest is quantum gravity, Mandelstam has in recent years made major contributions to the elucidation of confinement, c.g., the analysis of the Schwinger-Dyson equations and the magnetic superconductor ansatz for the QCD vacuum.

\section{Hadron Spectroscopy}

Having analyzed the fifteen-year experimental record of the "E" meson, Chanowitz argued that two different states were being confounded, one a $\mathrm{J}^{\mathrm{P}}=1^{+} \overline{\mathrm{q}} \mathrm{q}$ meson and the other a $\mathrm{J}^{\mathrm{P}}=0^{-}$glueball. He and Sharpe have used the bag model to study the spectrum of "meiktons" (mixed mesons made of $\bar{q} q \mathrm{~s}$ ). Sterling has considered some unsettled questions in glueball phenomenology, such as the inconsistencies in the bag model formulation of a glueball.

\section{S-Matrix}

Chew, Stapp, and collaborators (Finkelstein, Levinson, McMurray, Espinosa, Poenaru) have pursued an ambitious program known as the "topological bootstrap." The goal is to recover the successes of the standard model of strong, weak, and electromagnetic interactions using $\mathrm{S}$-matrix ideas in a topological framework. They have recovered the conventional particle spectrum, with a prediction of just four generations and an order of magnitude estimate of the fine structure constant. Work has continued to see if other features of the standard $S U(3)_{\text {color }} \times S U(2)_{L} \times U(1)$ model will also emerge.

\section{Weak Interactions}

Suzuki, with Shrock, has studied models in which the Higgs boson has predominantly unobservable decay modes, thus drawing attention to the importance of inclusive searches. DeForcrand has used the $K_{L}-K_{S}$ mass difference to obtain a bound on a right handed gauge boson that is insensitive to the mass of the right handed neutrino. Axelrod has studied $Z \rightarrow \vec{t} c$ as a means of detecting the $t$ quark if $M_{Z}$ is below the $t t$ threshold. Hung, with Bjorken and Buras, has exam- 


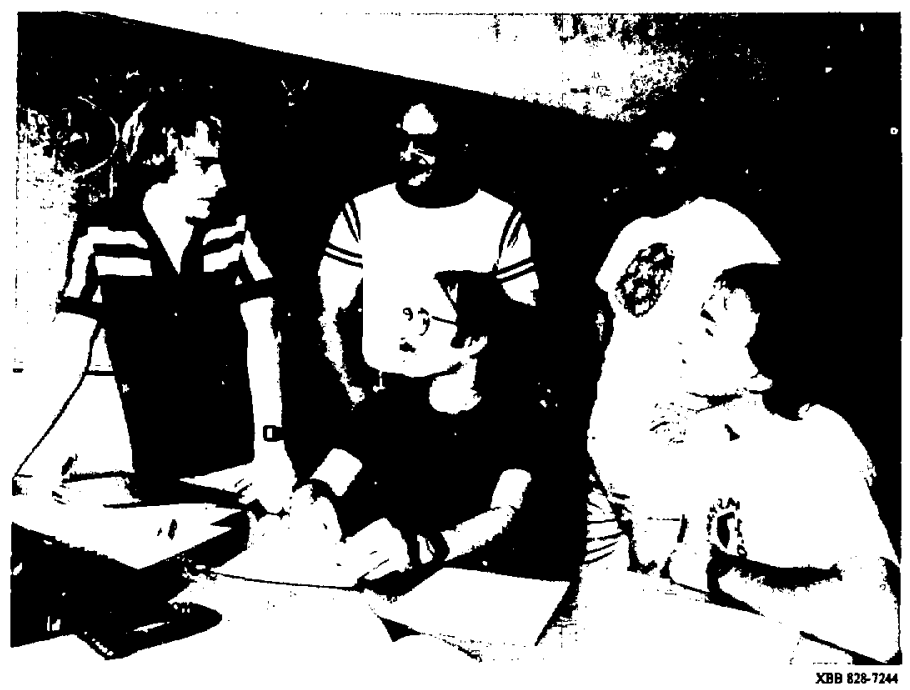

Fig. 24. Discussion on grand unification in the Theoretical Physics group. Ian Hinchliffe (left) makes a point to (clockwise) Professor Martin Halpern, graduate students Matt Visser and Stephen Sharpe, and Michael Chanowitz.

ined the possibility of a "petite unification" of weak and electromagnetic interactions at sub-GUT energies. Cahn, with Harari, has systematically compared the sensitivity of a variety of experimental processes as probes of flavor-changing "horizontal" gauge bosons.

\section{Quantum Gravity}

Mandelstam has investigated the relationship between quantum gravity and the dual string model. Gaillard and Zumino, with Ellis, have been involved in constructing an "ultimate" supergravity theory, based on $\operatorname{SU}(8)$, that would unify all the known forces and explain quark-lepton matter as composites of elementary "preon" supermultiplets.

\section{Cosmology}

Hung, with Nussinov, has considered the effects of heavy Majorana neutrinos on the evolution of the very early universe, such as formation of blauk holes with subsequent increased annihilation rates for monopoles. Greensite and Snyderman have looked for a solution of Einstein's equations in higher dimensions which has a "Koluza-Klein epoch," with 4 "normal" dimensions and $\mathrm{n}-4$ compactified dimensions.
Publications

Classical Solutions and the Large N Limit. K. Bardakci, Nuct. Phys. B 378, 263 (1981): UCP-PTH-80/8.

Dominant Euclidean Configuration for all N, K. Bardakci, D. Caldi and H. Neuberger, Nucl. Phys. B177, 233 (1981); LBL-10885.

Bianchin Identity, Strong Coupling Expansions and Duality for Abelian Lattice Gauge Theory, G. Batrouni, submitted for publiation in Nuclear Physies B; LBL-13424.

Impact Piclure Description of High Energy Elastic Proton-Proton Polarization, C. Bourrely, submitted for publication in Physical Review D, LBL-13490.

Chemizal Signatures for Superheavy Elementary Particles, R. Cahn and S. Glashow, Science 213, 607 (1981); LBL-12010.

Anomalies and the Particle Content of Grand Unified Theories, R. Cahn, Phys. Lett. I04B 282 (1981); LBL- 12379.

The Hierarchy Problem in Supersymmetric Grand Unified Field Theories, R. Cahn, L. Hall and I. Hinchlifte, accepted for publication in Physics Letters B; LBL 13726. 


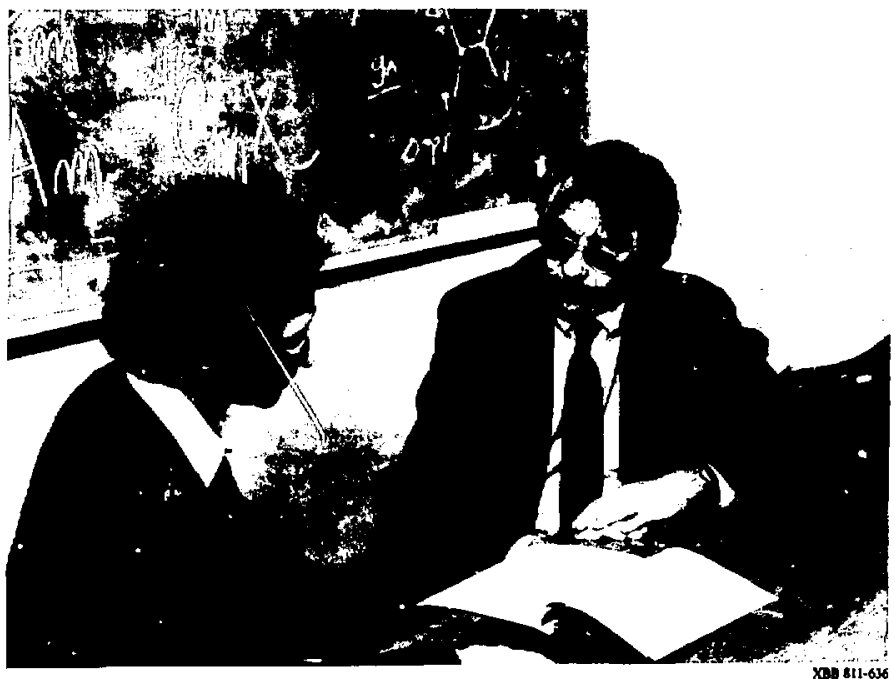

Fig. 25. Robert N. Cahn (left) and Professor Sheldon L. Glashow of Harvard discuss a common research project during Glashow's visit in the summer of 1981. A modest summer program brings such distinguished visitors to the Laboratory for mutually productive interactions.

Have We Seen Our First Gluebatl?, M. Chanowitz, Phys, Rev. Lett. 46. 981 (1981); LBL- $\$ 1977$

Glueball and Exotic Meson Candidates, M Chanowitz, in the Proceedings of the 1981 Mecting of the Division of Particles and Fields of the American Physical Society, University of California at Santa Cruz. Sepl. 911. 1981; LBL 13398.

Meson Spectroscopy: Quark States and Glueballs, M. Chancisitz, in the Proceedings of the SLAC Summer Institute on Particle Physics, July 27-29, 1981; L9L-13593.

A Topological Theory of Electric Charge, G. F. Chew, J. Finkelstein, R. McMurray, Jr. and V. Poenaru, Phys. Lett. 100B, 53 (1981); LBL-11431.

Topological Bootstrap Theory of Hadrons, G. F. Chew and V. Poenaru, Zeit. fur Physik C11, 59 (1981); LPL-11433.

Topological Theory of Electramagnetism, G. F. Chew, J. Finkelstein. R. McMurray, Jr. and V. Poenaru, Phys. Rev. D24, 2287 (1981); LBL-J1435.
Zero-Entropy Bootstrap and the Fine Structure Constant, G. F. Chew, Phys. Rev. Lett. 47, 764 (1981); LBL 12436.

Baryon Magnetic Moments from Topological Theory, G. F. Chew and J. Finkelstein, Phys. Rev. D24, 3335 (1981); LBL-12579.

Topological Theory of Electroweak Vector Bosons, G. F. Chew, J. Finkelstcin, and V. Poenaru, Phys. Rev. D24, 2764 (1981); LBL 12588.

Topological Theory of Elt:mentary Hadron Coupling-Constants, G. F. Chew, J. Finketstein and M. Levinson, Phys. Rev. Lett. 47, 767 (19B1): LBL-1275B.

The Topological Bootstrap, G. F. Chew, Lalk presented at the Symposium to honor Francis Low on his 60th Birthday, Massachusets lnsti. tute of Technology; LBL-13276.

A Smooth Topological Pug for "Quark-Color" Switching, G. F. Clrew, submitted for publication in Zeilschrifn fur Physik; LBL-13322.

Topological "Vector Gluons", G. F. Chew and J. Fiakelstein, submit- 
ted for publication in Zeitschrift fur Physik; LBL- 13382

A Model-Independent Limit on the Mass of the Right-Handed Boson, P. deForcrand, submitted for publication in Physics Letters B; LBL13594.

F Meson Production in $\mathrm{e}^{+} \mathrm{e}^{-}$Annihilation, C. Flory, Phys. Lett, 99B, 365 (1981); LBL-11343.

Non+Perturbative Ellects in Heavy Quarkonia, C. Flory, Phys. Lett. 101B, 98 (1981); LBL- I 1551.

Non-Perturbative Calculation of the Heavy Quark Potential, C. Flory; LBL 12385.

Topies in the Theory of Heavy Quark Systems, C. Flory. Ph.D. thesis; LBL-1255B.

On the Width $x(3.55) \rightarrow \psi 3 \pi$, C. Flory and 1 . Hinchlifie, Phys. Lete. 107B, 139 ( I98I): LBL- 12703.

An Approach to the Unification of Elementary Particle Interactions, M. K. Gaillard, talk presented at the International Symposium in Memory of Werner Heisenberg, "Unified Theories of Elementary Par. ticles: Critiesl Assessment and Prospects". Munich, July 1981; LBL13371.

Higher Order QCD Corrections to Double Moment Rat: xs in Deep Inelastic Seattering, H. Haber, I. Hinchliffe and J. Sheiman, Nucl. Phys. B183, 397 (1981); LBL-11577.

Small $\tilde{\theta}$ and an Invisible Axion as Automatic Consequences of Grand Unification, L. Hall, in the Procedings of the 1981 Banfi Summer Institute on Particles and Fields: LBL-13328.

Large N Factorizing Configurations, M. Halpern, Il Nuovo Cimento G1A. 207 (198I): UCB-PTH-80/3.

Large N Matrix Mechanies and Constrained Classical Systems, M. Halpern, Nucl. Phys. B1B8, 61 (1981); UCB-PTH-80/10.

Large N Classical Solution for the One Matrix Model, M. Halpern and C. Schwart2, Phys. Rev. D24, 2146 (1981); UCB-PTH-81/5.

Photon and $\pi^{0}$ Production at the FNAL $\bar{p} p$ Collider, I. Hinchliffe and R. Kelly, Fermilab Internal Nole; LBL-12274.

Perturbative QCD, I. Hinchliffe, talk presented at the 1981 Meeting of the Division of Particles and Fields of the American Physical Society, Sunta Cruz, California, September 9, 1981; LBL-19351.

The Structure of Neutral Currents, P.Q. Hung, A un. Rev, Nucl. Part. Sci. 1981: 375-438; LAL-12364.

Petite Unifieation: An Alternative Viewpoint, P.Q. Hung, in the Proceedings of the Second Workshop on Grand Unification, University of Michigan, Ann Arbor, Michigan, April 2426, 1981; LBL-12826.
Higss Phase in Non-Abelian Gauge Theories, O. Kaymakcalan, Ph.D. thesis; LBL- 12840.

Finite Size Wilmon Loops, J. Kowall and H. Neuberger, Nucl. Phys. BiB9, 535 (1981); UCB-PTH-8I/2.

Finite Size Wilson loops in Z(2), J. Kowall and $H$. Neuberger, Phys. Lelt. 106B, 197 (1981); UCB-PTH-81/3.

Spacelike Wilson Loops and Roughening, J. Kowall and $H$. Neuberger, submitted for publication in Physical Review D; UCB-PTH$81 / 6$.

Feynman Path Integrals in Large Number of Dimensions, J. Kowall, Phys. Rev. D24, 3329 (1981); UCE-PTH-81/7.

Flavor Symmetry in Nonleptonic Decays of Bottom Mesons, W. Lin. submitted for publication in Zeitschrift fur Physik, LBL-12270.

Renormalization in a Spontaneously Broken Case, S. Naito, submitted for publication in Physical Review D; LBL-13414.

Nonperturbative Contributions in Models with a Nananalytic Behavior gt Infinite N, H. Neuberger. Nucl. Phys. B179, 253 (1981); UCBPTH-BO/9.

The Three Je! Angular Distribution-A Detailed Test of $\mathbf{C D}, \mathrm{S}$. Shatpe, Phys. Lett. 106B, 331 (1981); LBL-13018.

A Gauge Invariant Multipole Expansion Scheme for Heavy QuarkAntiquark Systems in Quantum Chromodynamics, K. Shizuya, Phys. Rev. D23, 1180 ( 1981 ); LBL-1 1004.

Soft-Gluon Effects in Nonleptonic Decays of Charmed Mesons, $K$. Shizuya, Phys. Lett. 100B, 79 (1981); LBL-11884.

Higgs Mixing and CP Violation, K. Shizuya, Phys. Rev. D23, 1613 (1981): LBL-12004.

Soft-Gluon Effeets in Semileptonic Decays of Charmed F Mesons, K. Shizuya, Phys. Lett. 10SB, 406 (1981); LBL-12830.

Soft-Gluon Effects in Charmed Meson Decays, K Shizuya, in the Procoedings of the INS Symposium on Quark and Lepton Physics, Tokyo, Japan, June 25-26, 1981; LBL-13222.

Spins and Baryons in the Topological Expansion Il: Baryons. H. Stapp. submitted for publication in Nuovo Cimento; LBL-11770.

On the Regular Holonomic Character of the S-matrix and Microlocal Analysis of the Unitary-type Integrals, H. Stepp, acopted for publication in Communications of Mathernatical Physies; LBL-12571.

Mind, Matter, and Quantum Mechanics, H. Stapp. acceptet for publication in Foundations of Physics; LBL-12631

Topological Thtonry of Hadrons I: Mesons, H. Stapp, submitted for 
publication in Physical Review D; LBL-13310.

Exact Solution of the Infra-red Problem, H. Stapp; LBL-136S1.

Nonleptonic Weak Decay Rate of Explicitly Finored Heavy Meson, M. Suzukj, Nucl. Phys. B177, 413 (19BI); UCB-PTH-80/4.

On Search for Heavy Hadrons through Jet Invariant Mass in Electron-Positron Arnihilation, M. Suzuki, Phys. Rev. D23, 195I (1981): LBL-12077.

Longitudinal Stability of a Particle Beam in the Presence of Resonant
Cevities, Part 1, R. Warnock; LBL-13180.

Duality Rotations, B. Zumino, tulk presented at the Nuffield Workshop on the Quantum Structure of Space and Time, ImperinI College, London, August 1981; LBL-13564.

Spontancous Breaking of Supersymmetry, B. Zumino, talk presented at the International Symposium in Memory of Werner Heisenberg "Unified Theories of Elementary Particles: Critical Assessment and Prospects", Max-Planck Institute, Munich, Germany, July 1981; LBL-1369I.

\section{Heavy Ion Fusion}

Successful designs of commercial-scale electric power production plants using heavy-ion-ignited jnertial fusion must satisfy the six-dimensional phase space volume condition; two aspects have been studied. (1) A detailed 1978 study applicable to if linac and synchrotron systems has been extended to include induction linacs; it shows these to have a distinct advantage. (2) In collaboration with LLNL, the relation between target requirements and the phase constraint has been developed; the reduction in target gain and/or increase in beam energy needed to ease this constraint are evaluated. In other work, partly with LLNL, requirements for significant spot heating experiments have been developed, and selection of induction linac test-bed designs that can meet them have been studied.

\section{Research Staff}

David Judd

\section{Publications}

Impact of the New Heavy-Ion-Fusion Target Requirements on the Phase Space Constraint. D. Judd, R. O. Bangerter. Joint LBL-LLNL Report, LNN report $1563_{\mathrm{B}} / 146 \mathrm{~g}$. LBL report HI-FAN-163, 1981.
The Influence of Tirget Requirements on the Production, Acceleration, Transport, and Focusing of Ion Beams. D. Judd, R. O. Bangerter, J. W-K. Mark, D. J. Mexker. Procedings of the Fourth International Topical Conference on High-power Electron and Ion-beam Research and Technology, Palaiseau, France, June 29-July 3, 1981, p. 601, H. J. Doucet and J. M Buzzi, ats., Ecole Polyrechnique, Palaiseau, France.

On the Possibility for High Temperature Physics Experiments Using Material Heated Directly by Energetic lon Beams. L. Judd, J. W-K Mark, D. Bailey, R. More, and S. S. Yu. Paper presented at 1981 Nuclear Explosives Design Physies Conference, October 27-30, 1981, LLL (Paper and conference classified.)

A Simple Expression for Third-Onder Geometric Aberration of Quadrupole Doublets. D. Judd. LBL Report HL-FAN 188, November 1981.

Target Design Considerations for the Production, Acceleration, Transport, and Focusing of Ion Beams. D. Judd, R. O. Bangerter, J. W. K. Mark. 1981 Leser Fusion Annual Report, Lawrence Livermore National Laboratory.

Proposed Disk Healing, Focusing, and Beam Transport Experiments for Acoclerator Test Facilities. D. Judd, W. M. Fawley, S. S. Yu, J. W-K. Mark, R. O. Bangerter. 1981 Lestr Fusion annual Report. LNL.

Phase-Space Constraints on Induction-Linac Heavy-Ion InertialFusion Igniters. LBL Report 14038, UC-21, 1982. 


\section{Stochastic Beam Cooling}

LBL is pursuing stochastic beam cooling-a technique used to increase the phase-space density of a captured batch of rare particles such as antiprotons, or, in a combined action, to continually cool and accumulate many batches into a single intense beam.

General equations were developed for the cooling process, including bunched beams, and beam electrode structures were analyzed. Those results are being applied in a collaborative effort with Fermilab to design a source of cooled antiprotons for the colliding beam project (TEV I). This is a project to upgrade Fermilab's Energy Saver to a 2-TeV collider with two experimental areas (the major one of which involves the CDF; see the Division's experimental physies program at Fermilab). To reach such unprecedented energies at good luminosities is an ambitious goal.

A major upgrade of the design flux of cooled antiprotons for the Fermilab collider was undertaken at mid-year and entailed a change from batch pre-cooler technology to an all-stochastic accumulator. Physics dcsign was redirected to match this more demanding task. The upgraded design will provide a high flux of cooled antiprotons. An 8-GeV accumulator ring will contain a complex cooling and stacking system with advanced, high-frequency cooling equipment. Frequent visits to Fermilab by LBL staff to discuss designs and goals led to our prcposal of a conceptual design based on operating frequencies of $2.4 \mathrm{GHz}$ and $4-8 \mathrm{GHz}$. LBL beam-transport specialists generated ring lattice arrangements to accommodate the evolving needs of the cooling system plan.

In other work, an analysis was completed of the response of a beam electrode in which the beam couples to a coaxial line through slots in the wall of the coax. This slotted coupler, a form of traveling-wave electrode, has been developed and used at CERN. To gain a general understanding of such electrodes, LBL theorists treated the case in which beam and wave may be of unequal velocities. This analysis provides a design guide and an evaluation relative to other electrodes.

Expressions for Schottky signal suppression for transverse and longitudinal cooling in the presence of band overlap were derived from a Vlasov equation approach. These results take into account the character of the feedback system in each harmonic band and the distinction between devices sensitive to energy or to frequisncy. The formulation predicts possible large-gain instabilities which correspond to coherent overdamping. The stability thresholds derived apply as well to any localized feedback system or impedance, and the analysis can be extended to any spatially varying impedance structure. For stochastic cooling, it appears that systems with energy-dependent gain variation are significantly less susceptible to such instabilities than are systems with substantial filter-derived gain variation.

Computer codes were developed to evaluate the signal suppression numerically and were introduced into existing Fokker-Planck equation solvers. Single-particle longitudinal damping with transversely varying gain was analyzed in the presence of betatron oscillations, and a relatively simple expression was derived for the effective gain.

Transverse and longitudinal Fokker-Planck coefficients have been derived for the time evolution of the phase-space distribution of bunched beams undergoing stochastic cooling, including amplifier noise and signal suppression effects. A general expression for signal suppression, including band overlap, is obtained. The analysis has been extended to coupled, threedimensional cooling and to nonlinear pickup-kicker devices. Analysis progressed to the point of numerical analyses of realistic cooling scenarios.

\section{Research Staff}

J. Bisognano, S. Chattopadhyay, A A. Garren, K J. Kim, L. Smith.

Publications

Stochastic Cooling. J. Bisognano and C. Leemann. Proc. 1981 Summer School on High Energy Particle Accelerators, Fermilab, Batavia, Illinojs, 1982. 


\section{COMPUTER SCIENCE AND MATHEMATICS}




\title{
COMPUTER SCTENCE AND MATHEMATICS STAFF
}

\author{
Carole Agazzi \\ Norman Albright \\ Rosemary Alen \\ Donald Austin \\ Ozalp Babaoglu \\ William Benson \\ John Boistad \\ Bruce Burkhart \\ Gloria Butler \\ Tonia Cantrell \\ Paul Chan \\ Alexandre Chorin \\ Phillip Colella \\ Paul Concus \\ Craig Eades \\ Susan Eggers \\ Fred Gey \\ Wayne Graves \\ Claude Greengard \\ William Greiman \\ F. Alberto Grunbaum \\ Ole H. Hald \\ Dennis Hall \\ Paula Hawthorn \\ Robert Healey \\ Valeric Heatlic \\ Harvard Holmes \\ Udi Hrushonski \\ Laura Johnson \\ Rowland Johnson \\ William Johnston \\ Robert Krasny \\ Peter Kreps \\ Ivy Kuo \\ Linda Kwok \\ Douglas Lanam
}

James LaVita

William Laubenheimer

Per lotstedt

Aaron Marcus

John McCarthy

Rita McLaughlin

Loren Meissner

Deane Merrill

Irma Miranda

Lesta Nadel

Michael O'Dell

Suzanne O'Del!

Frank Oiken

Mirta Perlman

Michac] Powell

Carl Quong

Steven Rosenberg

Victor Roytburd

Susan Sacks

Debbie Seherrer

Esther Schroeder

James Sethian

Arie Shoshani

Gregory Sivashinsky

Alan Smith

Julia Snyder

Lawrence Stark

Joseph Sventek

Virginia Sventek

Lester Tabata

Audrey Tam

Enrique Thomann

Philip Wing

Herry Wong

Alber Yen 


\title{
COMPUTER SCIENCE AND MATHEMATICS
}

\author{
Introduction
}

The Computer Science and Mathematics (CSAM) Department conducts basic and applied research in Mathematics, Computer Science and Statistics. The mathematics work centers on solutions of partial differential equations with respect to turbulence theory, multiphase flow through a porous medium, and combustion theory. Statistics research focuses on the analysis of ecologic data: data that exists in summary form. The problems addressed are the development of statistically valid data extrapolation and projection techniques. The computer science research addresses four areas: data management, graphics, distributed systems and networks, and software engineering.

Funding for basic research is provided by the Applied Mathematical Sciences (AMS) research program and the Office of Health and Environmental Research (OHER) in the Office of Energy Research, DOE. Funding is also provided for research, development and demonstration projects by other federal agencies, including the U. S. Department of Labor Employment and Training Administration, the Army Corps of Engineers, the Defense Nuclear Agency, and the Defense Advanced Research Projects Agency. The combination of basic research with practical prototype projects and applications provides a base for innovative solutions to research problems in information systems development, computer networking, epidemiological studies, and the application of numerical methods to energy technology. During 1981, the projects undertaken included continued demonstration and development of a distributed information system containing extensive socio-economic, environmental, and demographic data (SEEDIS/DCN); design issues for a seismic data center (DARPA Center for Seismic Studies, CSS); and upgrade of research computing facilities supporting the department's basic research.

The CSAM staff is comprised of over 50 computer scientists, mathemsucians, engineers, computer technicians, and administrative personnel. UC Berkeley and UC San Francisco faculty, post-doctoral appointecs, and graduate students are an integral part of the research stafi. Visiting scientists participating in the research program included Mantania Ben Artzi of Israel Institute of Technology, Haifa; Zen Chen of the National Chiao Tung University, Taiwan, China; Gregory Sivashinsky of Tel Aviv University, Israel; and Zhen-Huan Teng of Peking University, China.

CSAM sponsors workshops, colloquia and seminars and provides supervision of graduate students in the masters and doctoral programs in applied mathematics, statistics, and computer science at UC Berkeley.

During 1981, the department hosted the first Workshop on Statistical Database Management, bringing together representatives from research laboratories, universities and industry working in this emerging specialization in data management. There were 100 participants at the workshop at Vallambrosa Conference Grounds in Menlo Park. As the department first became involved in design issues for the DARPA Seismic Data Center, a workshop was held to formulate research issues, define support requirements and establish working groups amongst the several support contractors. Other meetir.gs hosted during 1981 included the annual Workshop on Distributed Data Management and Computer Networks, the annual meeting of the DOE Numerical Analysis Special Interest Group, a meeting of the ANSI committee on Fortran Standards, and several local user group meetings of DECUS (the Digital Equipment Corporation Computer Users Group.) Professional outreach activities included special participation by members of CSAM staff in standards committees working groups and as conference referees.

Paul Concus serves on the Editorial Board of the SIAM Journal of Scientific and Statistical Computing, is a member of the Board of Trustees, Mathematical Sciences Research Institute, and organized the Second International Colloquium on Drops and Bubbles, held in Monterey, November 1981.

Bill Johnston participates on the ANSI X3H3 Technical Committee on Computer Graphics Programming Languages.

Loren Meissner served as Secretary of the ANSI X3J3, Fortran Standards Committee.

Deborah Scherrer serves on the USENIX (UNIX Users' Group) Board of Directors.

Arie Shoshani is Associate Editor of ACM Transactions of Database Systems

Arie Shoshani and Paula Hawthorn served on the program committee for the ACM Special Interest Group on Maragement of Data (SIGMOD) Conference.

Joseph Sventek served as chairman of the DECUS Bay Area RSX-11/IAS Local User Group (BAYLUG) and as a member of the Steering Committees for DECUS RSX-11/IAS and struetured languages SIC. 


\section{Mathematics}

Research in mathematics includes the areas of applied analysis, computational mathematics, and numerical methods for partial differential equations. The staf also provides consulting services to LBL staff members and supervises the Computer Center mathematical software library.

\section{Applied Analysis}

\section{Capillarity Phenomena}

The work in capillarity phenomena is concerned with questions of the existence, stability, and qualitative behavior of solutions of the governing nonlinear elliptic partial differential equations. Work on the capillary free-surface problem continued. New analytic and computational results were obtained for the discontinuous behavior of free surfaces in domains with very general cross-sections. It was proved that under certain conditions a solution surface can become singular over an entire subdomain as criticality conditions for existence are approached. This situation is in contrast to the one for the wedge phenomenon, studied previously, for which the singularity occurs only at a boundary point. Formulation of low-gravity physical experiments for confirming the mathematical results continued.

\section{Inverse Problems}

Reconstruction of the Density of the Earth from Free Oscillation Data

This work uses the frequencies of the fundamentals and overtones of the earth's free oscillations to determine the density and velocity of the waves in the upper mantle. In the last year two algorithms were developed that solve numerically the inverse problem of the reconstruction of the density of the earth from free oscillation data. The first algorithm uses frequency data from two torsional spectra, the second uses one spectrum plus the vclocity of the $S$ wave. The algorithms are based on the Rayleigh-Ritz method but involve several unusual features. For example, a nonstandard inverse eigenvalue problem is produced and solved by a contraction method that uses a repeated change of basis functions. The numerical experiments show that the algorithms give a stable reconstruction of the density (and the velocity) in the upper mantle and that the overtones with the lowcst angular order lead to the most accurate results. Finally, the experiments reveal that overtones with various angular orders can be used simultaneously in the reconstruction of the density.

\section{Image Reconstruction from Projections}

This work is directed toward developing optimal numeitical schemes and understanding the mathemation constraints on different forms of tomography, with spe- cial emphasis on the effects of limiting the number or range of the directions defining the projections. The problem of optimal choice of the directions on the sphere for the purpose of reconstruction of a function from plane integrals has been investigated. It has been verified experimentally that choosing the directions to be the equilibrium configuration of an array of $\mathrm{M}$ electrons attached to the surface of the sphere gives close to an optimal choice for $M$ directions. The problem of computing the spectral decomposition of an integral operator has been studied for geometries more complicated than those previously reported. This problem is made numerically manageable by producing analytically a second order differential operator that commutes with the integral one. The classical results cover the Euclidean case but the tomographic application deals with functions on the sphere. A startling connection has been found between geometries for which such operators exist and special solutions of the Korteweg-de Vries equation. The problem of numerical inversion of the Laplace transform has been found to be amenable to a similar treatment.

\section{Computational Mathematics}

\section{Elliptic Partial Differential Equations}

Work was completed on a hybrid integral-equation fast-direct technique for solving two-dimensional Poisson and biharnonic problems on irregular domains with smooth boundaries. The boundary is embedded in a rectangular region, and a discontinuous extension based on ai integral equation formulation is used to obtain a procedure amenable to fast direct methods. Work continued from our previous development of preconditioned conjugate gradient methods for solving the sparse systems of equations arising from two dimensional elliptic problems. Extensions suitable for three-dimensional problems are being considered.

\section{Numerical Methods for Partial Difierential Equations}

\section{Vortex Dynamics and Turbulence}

Research in vortex dynamics and turbulence concerns numerical methods for low-Mach-number highReynolds-number flow in two and three dimensions. Of 
central interest is the vortex method, which represents vorticity as discrete elements that are transported within the fiuid. The coalescing and stretching of the vortex elements in a computation simulate in a natural, highresolution manntr physically observed fluid motion.

The major developments in vortex dynamics in 1981 were the development of an efficient threedimensional program for the analysis of periodic turbulence and the first combination of a vortex method with a renormalization group procedure in order to analyze generic properties of turbulence. The small scale structure of turbulent incompressible flow has been formulaied in terms of invariance of the flow under certain scaling transformations. A set of computer programs that calculate the scaling law and verify that the computed solution satisfies it were developed. These programs have shown that the Hausdorff dimension of the carrier of vorticity is approximately $5 / 2$, the Euler equation breaks down in finite time in three-dimensional flow, and the temporal intermittency is much larger than previously thought. A theory of vortex methods was developed and concludes that higher order accuracy can be achieved by altering the form of the cut-off without additional computational expense.

\section{High Resolution Methods for Hyperbolic Equations}

Research in high resolution methods for hyperbolic equations concerns the development of accurate and robust numerical methods for problems whose solutions have discontinuities. These methods are based on the incorporation of analytical solution elements into the numerical algorithms. Practical applications are to gas dynamics, flow through porous media, and combustion.

Better methods have been developed for solving problems in more than one space variable. These include methods based on the second order Lagrangian Godunov method introduced by van Lee and a two-dimensional front tracking scheme. A single-step formulation of van Leer's scheme was developed for the case of Eulerian hydrodynamics and other higher order extensions of Godunov's method for gas dynamics. These methods have the property that discontinuities are transported with little or no spreading and without loss of accuracy in regions where the solution is continuous. The front tracking scheme overcomes some of the difficulties associated with fractional splitting for porous flow problems and is based on the algorithm of Noh, Woodward and Chorin that transports cell fractions at a front.

Better algorithms for solving the Riemann problem for more complicated systems were derived. Some of these shed new light on the problem of transition from deflagration to detonation. These algorithms were used for calculating multiple Mach reflections in nitro[en, for non-Cartesian zeometries, and for gas dynamics with a burning front, which could either be a deflagration or a detonation. The algorithms have been evaluated in the context of the random choice method.

In following the position of shocks or steep gradients that are not known in advance, nonuniform grids that change in time and adapt to the solution have been found useful in some cases. An adaptive mesh refinement method was implemented and partially analyzed for the initial boundary valuz problem for a first order hyperbolic system in one space dimension.

\section{Combustion Modeling}

The research in combustion is concerned with the accurate calculation of possibly turbulent fluid flows in the presence of large energy sources due to chemical reactions. This work utitizes the vortex and high resolution numerical methods coupled with numerical techniques for the exothermic reactions.

Several new methods were combined to produce a numerical model of turbulent combustion that agrees well with experiments. This model used a vortex method including blobs and sheets to model the turbulence, a Huygens principle to model the propagation of the flame, and a new source algorithm based on high activation energy asymptotics to describe the exothermic effects of combustion. The combination of numerical and asymptotic methods allows the usual limitations of asymptotic methods to be transcended, in particular, it need not be assumed that the energy release is small.

A global constructive existence proof was produced for fronts whose dynamies are given by propagation normal to themselves at a scalar speed. One consequence of this construction is that flame fronts are globally stable. Cusps in the flame front are formed due to linear instability and are overtaken by the front due to the propagation of the front normal to itself. This process is readily observed in photographs of flames. Analysis also indicates that tracking techniques based on representing a flame as a globally defined smooth curve will fail due to the successive formation of cusps. A perturbation theory was developed and explains the formation of Mach stems in reacting shock fronts, a previously unexplained phenomenon.

Various numerical methods for calculating reacting fluid flows have been compared. The random sampling method for computing the solutions of one dimensional reacting gas flow problums is first order accurate, provides sharp resolution of jiscontinuities, but is not conservative. Also, at each step the complete Riemann problem for detonation initial data may have to be solved, which is a difficult and unfamiliar programming problem. Other recent work has shown that difference methods can provide poor computational acceracy upwind from the detonation front and are unaole to resolve the qualitative features of detonations with large reaction rate and small heat conduction coefficients. 


\section{Mathematical Software and Consulting Services}

Research in scientific graphics focuses on the mathematical questions arising in the graphical display of the numerical solutions of partial differential equations. The subject areas of interest include the representation of three-dimensional turbulent fluid flows, the visualization of flame propagation in combustible fuels, and the representation of internal density variations within three-dimensional objects.

A compuler program was written to provide interactive color graphics for combustion simulations. The user can display the velocity field of the fluid and the location of the flame front, can skip ahead or back in time, can zoom in on a particular section of the flow, and can compare the results of several different comr puter runs simultaneously. Work on the problem of visualization of three-dimensional turbulence has begun. An interactive program that provides full threedimensional sotation and perspective viewing of particle paths has been written. Another interactive program has been written to display the results of a numerical simulation of the evolution of a turbulent vortex in three dimensions.
In addition to its research activities, the Mathematics Group supervises the management of the Computer Center software library, which covers essentially all major areas of contemporary numerical methods. In connection with this activity, consulting services to Computer Center users is provided in numerical mathematics, applied analysis, and statistics.

During the past year the major accomplishment was the completion of an integrated catalog of all the mathematical software available at the LBL computing center. This is now incorporated in the WRITEUPS subset LIBRARY. It allows users to locate desired programs easily and efficiently. The standard mathematical libraries (International Mathematical and Statistical Library and Numerical Algorithms Group Library on the CDC and VAX machines, and the National Physical Laboratory Optimization Library on CDC) were updated. The new libraries FISHPAK for elliptic partial differential equations, AMOSLIB for special functions, the BSPLINE routines from de Boor's Practical Guide to Splines, the MINOS package for large sparse optimization, and PDECOL for time-dependent partial differential equations were installed.

\section{Computer Sciznce}

\section{Data Management}

Many applications relevant to the DOE mission require the management of databases that have unique characteristics calling for the development of special data management techniques that are not readily available in commercial systems. The purpose of the data management research program is to identify problems of managing such databases, termed "statistical databases," and to develop techniques that specifically address these problems. The research program has proceeded with complementary activities in three areas: data modeling, enhanced user interfaces, and efficient data compression and access. In the area of data modeling the emphasis is on developing models for summary sets, directory structures for metadata (data about the databases), and neutral internal models to facilitate a multiplicity of user interfaces. User interfaces are designed to simplify user access, especially for users who are not experts with computer usage. Efficient data compression and access is achieved by taking advantage of the special characteristics of statistical databases. Our research includes compression techriques designed for fast access, and a database machine to support compression and partitioning operations for data management systems.

\section{SUBJECT: A Directory-Based System}

SUBJECT is a system for organizing and accessing statistical databases based on an acyclic directed graph structure. The main tasks accomplished during 1981 were the documentation of, and experimentation with, the SUBJECT prototype system. The prototype system includes the most essential modules needed for experimentition. This includes the data structures to support the SUBJECT graphs, and the software to browse, index and search these graphs.

Four large scale application systems were modeled:

a) SEEDIS - Socio-Economic Environmental Demographic Information System, developed at LBL. SEEDIS contains a variety of databases (including the 1970 census) that are geographically besed. 
b) DRD - Data Resource Directory, a systen for supporting energy data on the production, con* sumption and distribution of energy sources. The development of this system is supported by the Energy Information Administration (EIA) of the Department of Energy.

c) ESARS - Employment Security And Reporting System; the ESARS Data Retrieval System was developed at LBL for the Department of Labor.

d) LMPM - Labor Market Projection Model, developed at LBL for the Department of Labor. It uses data from previous years to project labor trends.

Experimentation with these systems led to the identification of several areas that requirc further research: physical dependence, different names for the same data element, same names for different data elements, and multìple referencing of tables.

\section{GUIDE: A Graphical User Interface for Data Extraction}

Another approach to providing an effective user interface is to use graphies techniques. GUIDE is a graphical query system designed to ease the task of query formulation. Often, statistical data bases have complex structures and the relationships between data items are not always clear. This problem is aggravated by the large number of terms that users have to remember: names of files and attributes, and formats of data values. In addition, the syntax of some query language has to be learned.

The problems described above motivated the development of both SUBJECT and GUIDE, and are in fact, common to user interfaces to large complex databases in general. GUIDE is designed to present information as a network of entities and relationships using color graphics. The main idea is to let a user pick the relevant part of the graph structure, and mark a path (using a different color) over it to represent a query. The specification of a path on the graph is similar to the concept of using path query languages, which take advantage of explicit links (or relationships) between entities.

The use of graphies for enhancing query formulation has been suggested before, especially for relational systems. However, in such systems the relationships between entities of the database are not explicit, imposing the burden on users to determine the relationships and express them in queries. GUIDE is based on the "entity-relationship" model, where relationships are represented explicitly. The display of information on the screen alleviates the need for the user to remember names of database objects, and the graphics path structures provide an alternative to query language expressions that use syntactic constructs.

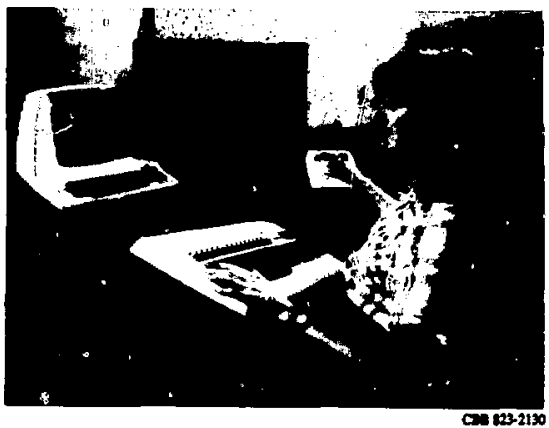

Fig. 26. GUIDE provides a graphics user interface for complex databases. The user formulates the database query by marking a path on the database structure shown on the screen to the right and responding to menu commands on right side of the graphics screen. The terminal to the right serves as the input device and they query result presentation device.

GUIDE provides. capabilities for "seeing" the database schema at various complexity levels. At the highest level only the entities that are most relevant to understanding the data are shown. Successive levels show more and more detail. The user can choose to concentrate on a specific node, move it to the center of the screen, and find relevant nodes and arcs around it. A hierarchical subject directory can be used to locate nodes of interest, and an attribute directory can be used to locate attributes for query specification. The use of directories is similar to their use in SUBJECT since they were found to be quite effective in guiding the user to the elements of interest. Partial results can be shown at any stage of the query specification, avoiding the frustration of incorrect queries that must be respecified.

\section{A Semantic Core Model for Statistical Databases}

The semantic core model serves as the middle interface between (high level) user interfaces and (low level) data storage and access. On the one hand, it should be rich enough in semantics to allow multiple data models to interface to it. On the other hand, it should be independent of any particular physice' struoture, so that it can interface to a variety of possible physical structures. This is particularly important for statistical databases, because they require unusual struotures such as transposed files, compression, and array linearization of cross products. 
User interfaces vary in style and complexity. They are usually based on a particular data model. Evidence with existing data management systems suggest that different models offer different advantages, which may be preferable to different users, even those using the same data base. The purpose of a semantic core model is to provide a neutral common basis for different data models and different user interfaces. It affords a single implementation of a data management system to which a variety of user interfaces can be interfaced. The structures of the semantic core model provide a formalism with direct application to the task of database design and specification.

An initial design and implementation of a protatype subset of the SCM was developed for simple schema definition and data dictionary management. This was motivated by a need to provide a framework for developing an abstract physical data model enconpassing special purpose statistical database structures (access methods) and by the need for an intermediate software interface between a high-level statistical query language under development and these access methods.

The prototype was developed on a Digital Equipment Corporation VAX $11 / 780$ running INJIX (VM/UNIX 4bsd). It was written in the language " $\mathrm{C}$," using YACC and LEX to develop the data definition grammar. Experience with this prototype, although strictly an experimental system, led to many ideas for extending and refining the definition language and basic concepts of the SCM, especially the distinction between declaration and binding, and the incremental approach to the definition process in general.

The constructs of the SCM were evaluated and refined on the basis of modeling portions of two real application environments. SEEDIS is a working system for accessing a collection of large geographic, socioeconomic databases typified by the U.S. Census. Database description, modeling and metadata management -emain difficult problems for statistical applications such a those contained in this information system. The ot ${ }^{\prime} r$ application studied is a seismic data center which is being developed to manage a heterogeneous collection of data. and support routine seismological analysis (earthquake detection and discrimination) and geophysical research. This typifies a scientific database application. Modeling these applications has provider insight into many of the higher level semantics built into the SCM.

\section{Compression Techniques for Statistival Databases}

The management of large databases requires data compression techniques 10 reduce the volume of data. However, data access usually suffers because of the need to decompress the data at the time of access. Most compression schemes require access time that is linear with the number of elements that have to be decompressed. Techniques have been developed for compressing statistical databases such that the access time required is logarithmic. Such techniques are possible because advantage is taken of the special characreristics of statistical databases.

In 1981 the general header compression scheme was developed, and a paper analyzing its performance relative to other more restrictive versions was presented at the Seventh International Conference on Very Large Databases. In anticipation of berchmarking the technique, a data loader and an access method for the basic version were designed and implemented. In addition to compressing the data, the loader also builds the header and its B-tree and allows a variable, vertical partitioning of the attributes.

A prototype for both the general and basic versions of the header compression scheme was designed. These particular versions of header compression were chosen for two reasons:

(1) First, they represent the extreme cases in the trade-off between functional capability and degree of compression and access time. The general scheme is on the more flexible end of the spectrum. It is capable of both eliminating multiple types of constants from the database and compressing each stored value to its minimal byte length. The basic scheme is the most restrictive of the variations. It can compress only one type of constant, and stores each value in the same number of bytes.

(2) Second, these two schemes have the widest applicability. Because of its versatility, the general scheme can be used to compress a wide range of statistical databases. The basic scheme has a wide applicability because the class of databases it compresses is so large. This class is comprised of two major database types. One is the large number of statistical databases that have a prevalence of only one constant, zero. The other is statistical databases that are ordered by composite keys ${ }^{l}$ whose values constitute an incomplete cross product. In these databases, the tuples of the incomplete cross product are stored, and the tuples with invalid composite key values are considereo the constant and are compressed.

The prototype design differs from that for the basic loader and access method previously implemented in that it incorporates features of the general scheme as well as the basic scheme in one modular system. Including both header compression versions in the same system takes advantage of many overlapping functions. The purpose of the prototype is to permii performance measurements of the data compression algorithm (i.c., access time and size of compressed database).

\footnotetext{
Keys that ene composed of ecreral attributes.
} 


\section{Microprocessor Assist System}

The Microprocessor Assist System is a databast machine design aimed at improving the costperformance ratio in statistical database systems. It is a microprocessor-based back-end system that performs a part of the work of a statistical data management system. The system consists of one or more trees of microprocessors that are at the bottom level connecled one to each disk (the leaf microprocessors) and at the highest level (the rool mictoprocessor) connected to the front-end computer. A single root microprocessor directs the activities of, and receives data from, its child processors. The tree can be more than two levels, and there may be more than one tree connected to the same front-end. However, for simplicity, in its initial implementation, a two-level single tree design is used.

The major functionality of the microprocessors is to implement compression and attribute partitioning techniques for the statistical data management system running in the front-end computer. Preliminary work during 1981 included performance studies of the design, where it is shown that statistical applications should perform well on such back-end systems, and a user survey to determine the work load characteristics of statistical database applications.

\section{Computer Graphics Systems}

The Computer Graphics Research Group in CSAM has research interests in several areas. The first area is that of data representation. Data representation is the general problem of how one displays a given type of data so that the important relationships are made evident by the graphical presentation. There are several on going projects in this area. In the CARTE project techniques are developed to display thematic information cartographically. In the CHART project, techniques are being developed for the graphical display of tabular information. Further, it is an ongoing concern of the graphics group to develop new techniques for the display of scientific information. Most of this work has been done in the context of the IDDS (Integrated Data Display System) project. In 1981 new work was in the areas of graphical user interfaces (described in the data management section "GUIDE"), distributed graphics systems, and the use and rendering of color.

\section{Network Distributed Graphics Systems}

The purpose of a basic graphics system is to provide a standardized set of functions to be used by applications that produce computer generated images. Important reasons for using such a system include: simplifying the development of applications, providing device independence, promoting application and programmer portability. Increasing numbers of applications relevant to the DOE mission require a graphics system capable of supporting structured pictures and complex types of interaction. The goal of research in this area is to develop a graphics system that meets these requirements while taking advantage of recent hardware developments, and incorporating the graphics work of the U.S. and international standards organizations, ANSI and ISO.

An important requirement for such a basic graph. ics system is the ability to support applications distributed across local area networks. Such networks make possible interprocess communication and file transfers between heterogeneous hosts running different operating systems. This enables the sharing of scarce resources, graphics equipment in particular, among prosesses running on different host computers. On the other hand, it also permits an application to reside on a host that bas special resources such as large databases, specialized computational hardware (e.g. array processors) or specialized, nontransportable software systems, but still make use of graphics hardware elsewhere.

Functions that a basic graphics systen must provide in order to support important applications, such as exploratory data analysis and picture editing, have been identified. This graphics system should make possible the following:

(1) Instancing: copying a complete image from one device to another (like a hard-copy button),

(2) Structured Symbol System: dynamic manipulation of graphical objects or symbols, in an application such as a picture editor. Hierarchy of depth greater than one will be required in order to provide the required capabilities,

(3) Local Transformations: local processing capability, in particular, local geometric transformations, should be available to the user in a deviceindependent way,

(4) Virtual Workstations: multiple logical view surfaces on a single device, which are so independent as to be allocatable to different processes, potentially running on different network hosts.

In addition, it must be possible to integrate the graphics system into a local area network; it must exist in an environment with multiple hosts and multiple operating systems and support many different types of graphics devices.

During 1981 the design of a network distributed graphics system which runs on the CSAM local area network was begun. The system will be instrumented to allow the gathering of performance data, and will incorporate several new ideas on the handling of attributes, color and raster primitives.

\section{Color in Computer Graphics}

The value of color in graphic display is potentially great. Because the human eye is several orders of mag- 
nitude more sensitive to variations in hue than it is to variations in monochromatic grey level, the use of color greatly enhances the communication of information.

Unfortunately, current methods of specifying and rendering color in computer graphics systems are inadequate. Even when specified in terms of a perceptual model, color is ultimately represented in terms of the hardware primary colors, usually red, green and blue. This representation, however, is not device independent. since these primaries are, in general, different from one device to another. Very significant differences have been found between color display devices and between display devices and film media when comparing identically specified images,

This situation is unacceptable in an environment with many different display devices and with applications requiring close control of color. Examples of such applications include: coloring schemes for bivariate choropleth maps, perceptually uniform color scales, and natural language models for color selection. Frequently a user wishes to preview on one device, an image which will ultimately be produced on another. Without a device independent method of color specification, many time-consuming iterations may be required.

During 1981 a series of experiments to determine the feasibility of using standardized color specification systems in conjunction with computer graphics devices were begun. Attempts were made to reproduce specific colors from the NBS color charts on a color video frame buffer using the monitor phosphor and alignment white CIE coordinates supplicd by the vendor. The results were disappointing; however, some insights were gained into the difficulties involved. For example two things were apparent: first, that successful reproduction of low intensity colors is sensitive to small perturbations in monitor characteristics, and second, that it is very difficult to determine the color coordinates of colors displayed on a monitor by comparison with standard color samples. This work will continue with the use of a spectroradiometer to make precise color measurements on the graphics devices and to develop techniques to establish the transformations from standardized color models to computer graphic devices.

\section{Software Engineering for Distributed Systems}

Distributed systems effort focuses on the design of distributed applications capable of utilizing a multiplicity of networks.

Despite the rapid increase in the number of computer networks, there has not been a corresponding increase in the number of distributed applications designed to exploit these networks, other than the canonical file transfer and virtual terminal protocols. This situation is especially prevalent when the hosts connected by a particular network are heterogeneous, i.e., consist of different hardware/operating system pairs. This situation will be exacerbated as the number of commercially available networks increases.

$A$ distributed application is nothing more than an implementation of a protocol layer on top of the process-to-process layer provided by the network, and can be visualized as occupying the application layer of the ISO open systems interconnection model. It is imperatjve that the distributed pieces of the application apply identical semantics to identical situations encountered during operation of the application to realize the robustness and freedom from dead-lock required of any protocol. Guaranteeing such an identical interpretation is generally a difficult and error-prone process, especially in heterogeneous environments.

The typical user-utility (man-machine) interaction can be viewed as a two-processor, distributed application over a fairly simple network (interactive terminal line). The use of virtual machine concepts has proven extremely useful in the implementation of these "distributed" applications in heterogeneous environments. The extension of these concepts to situations where a set of processes replace the human client should prove equally useful when designing more traditional distributed applications. Two primary benefits accrue from this approach:

- The same source code is portable to all of the systems. This guarantecs that the same functionality and interface is presented to users on each of the systems.

- By isolating the access to critical resources in the virtual machine primitives, the most efficient access possible can be guaranteed for any software utilizing the resource through the virtual machine.

The first test of the concepts described above was the design and implementation of a distributed, electronic mail system. The major premise of this work is that the adaptability issues can be addressed by extending the virtual machine concepts. The resulting portable modules provide the following benefits:

- The software is adaptable to changes necessitated by changing needs and/or standards. New features can be added to the source once, with all hosis on the internet bentiting from the change. The ease with which these simultaneaus updates can occur permits the use of the code as a "software breadboard" upon which new features can be implemented and tested in an heterogeneous network environment before they are included in a standard. Such experimentation is highly desirable, and not available in the normal implementation scenarios.

- Since all systems run identical implementations, 
the service level is consistent throughout the interret. This expedites the implementation of higher-level applications using the mail system as a network protocol layer.

The initial implementation using the virtual machine concepts is completed and in production use. The delivery system currently employs three different network substrates (DECNET, UUCP and LBL's Hyperchannel protocol) for interhost message trafic. The adaptability of the source code has been instrumental in the implementation and maintenance of the system. The portable source code is operational on several operating systems (RSX-1IM, VMS, UNIX). The standards upon which the system is based bave changed several times in the last year, the portability of the source code permits a drastic reduction in the effort to conform to the revised standards.

Of even greater importance, the extended virtual machine concepts have permitted additional network support in a straight-forward manher. Templates exist for sender and receiver processes to move the messages over a particular network, requiring only that five network-specific primitive functions be implemented. The effort required to support a new network substrate is approximately one stafi-day.

The system is currently serving as a testbed for the development and testing of other aspects of mail systems. Of special interest is security of the mail during transport and after delivery. Several methods for securing the messages are being studied, and implementation will oceur as required.

\section{Statistics}

\section{PAREP}

The Populations at Risk to Environmental Pollution (PAREP) project focuses on methodologic problems surrounding the use of ecologic data, and investigation of the influences of environmental pollution in the human disease process. The tools and data in SEEDIS provide the basis for testing statistical methodologies and epidemiological hypotheses, and enhancements and data additions to SEEDIS were made in response to needs of the PAREP project.

Work continued on a number of specific epidemiologic investigations including an investigation of mortality for ten selected causes of death, a study of melanoma incidence and income in the Third National Cancer Survey, and a study of cancers of the gum and mouth. Statistical methods and techniques studied included a detailed investigation of the methodological problems of multiple tegression analysis as applied to the study of air quality and monality. A methodological alternative to ecologic regression analysis of mortality rates was described and tested. An investigation of the validity of the data and assumptions used to estimate air quality for counties, census tracts, and other geographic entities was begun in 1981.

Enhancements to the information system base (SEEDIS) included:

- an interactive modeling capability for estimating air quality at any desired geographic level, from user-supplied input parameters;

- definition of new geographic levels (including all levels of the 1980 Census):
- enhanced aggregation and disag. egation capabilities, with specific attention to differences between 1970 and 1980 census counties;

- addition of geographic base map files to include 1980 census counties, 1970 census tracts (completing work begun in 1980), 1981 Standard Metropolitan Statistical Areas, and others;

- implementation of SEEDIS in batch mode, permitting night-time execution of large tasks for PAREP applications;

- experimental enhancements to the CODATA tools to include additional DDF (data description file) elements (this preliminary work is being generalized and greatly expanded in 1982).

- development of software to automatically revise SEEDIS data documentation to reflect new enhancements and data additions.

PAREP-related data installed in SEEDIS include:

- 1969-1977 leukemia mortality for whites by county, age, sex, race, year, and histology type;

- 1970-1977 population by county, age, sex, race, and year;

- 1968-1972 and 1973-1976 age adjusted mortality, by county, age, sex, race, and cause of death;

- preliminary 1973-1977 cancer incidence data for whites by tract, age, sex, cancer site and histology type, for areas covered by the NCl (National Cancer Institute) SEER (Surveillance, Epidemiology, and End Results) program; 
additional selected 1970 census data for whites by tract, age, and sex;

- 1974-1976 air quality data for sulfur dioxide and nitrogen dioxide, with 1-hour and 24-hour sampling intervals combined;

- 1970 population and household counts and population centroids for the ED/BG's (enumeration districts and block groups) of the 1970 census;

- 1980 population by race (PL94 reapportionment file) for ail geographic levels defined by the 1980 census;

- 1980 Census Summary Tape File IA for Rhode Island (other states became available in 1982).

Documentation of the SEEDIS system and PAREP databases was greatly revised and extended. These documents, a component of SEEDIS, can be browsed interactively or printed off-line. PAREP activities included presentations and distribution of documentation to collaborators and potential SEEDIS users. With regard to PAREP-related environmental health studies, the most important liaisons include the University of California School of Public Health, the Environmental Protection Agency, the National Cancer Institute, and the Northern California Cancer Program.

\section{Labor Market Projections Model}

The aim of the Labor Market Projections Model (LMPM) is to provide estimates of current socioeconomic conditions at the local level, for the entire nation. These estimates of the current local labor markets and population statistics are produced using a uniform methodology for the entire nation. However, it is possible to integrate locally developed data into the model in order to take advantage of analysts' knowledge at the local level. The LMPM project was developed in collaboration with the Employment and Training Administration of the Department of Labor to provide a comprchensive modeling system to be used by local CETA analysts. Two models for estimating current local needs have been developed. The first modeling method. which utilizes Current Population Survey (CPS) data, provides economic indicators for the population in general as well as demographic population breakdowns. The second method uses a refined cohori-component method to project population, labor force and unemployment for each geo-area of interest. This involves the separate prujection of births, deaths and net migration to give population by race by sex by five-year age cohorts for a particular area. Submodels are used to handle the military and collegiate populations separately

Substantial improvements were made to the population projections model: enhancements were made to the underlying methodology, new data from the 1980 Census was installed in the model, and the format of the resultant print-outs was improved.

1) 1980 Census data on the population by age at the state level was received and compared with the 1980 jopulation projections as calculated by the LMPM projections model. The model was run twice: once using the new migration submodel as memtioned above. and once with the commonly used plus-minus adjustment technique.

Figure 27 shows the results of this comparison. The first two columns indicate the mean absolute percent error over all age groups. The two remaining columns present the percent error for the age group having the greatest relative difference between the projection and the census result. The mean absolute percent error of the migration model is less than that of the plusminus technique in all but. two states. Furthermore, it is usually less by a factor of two. Looking at tite maximum error, again the migration model outperforms the plus-minus technigue and usually by a factor of two. For several states, Illinois, Louisiana, Montana, New Hampshire, New Jersey, and Texas, the percent error of the worst-fitted age group using the migration model is less than the mean absolute percent error using the plusminus technique.

Further testing of the migration submodel will be done as more Census data becomes available.

2) Several routines were written to force the sum of the population over each race/sex/age cohort in substate areas to add up to the corresponding $\mathrm{race} / \mathrm{sex} /$ age cohort at the state level. They work well and converge rapidly to a solution even when forcing the race/sex/age cohorts in over 25 substate areas to add up to the corresponding figure for the race/sex/age cohort of the state. Once the projections have been successfully calculated for the largest substate pieces, the program subdivides these pieces where desired using the just calculated race/sex/age projections for each piece as control totals.

3) Work was done to investigate the desirability of adding a third time interval to the projections. For the past few years, the projections model has projected in two time intervals: first from 1970 to 1975, and then from 1975 to 1980 . The desired projections for 1981 or 1982 were then obtained by applying the 1981 or 1982 control total to the projected 1980 population distribution. It was felt that this introduced fewer errors than trying to project to 1985 and then interpolating between 1980 and 1985 to obtain 1981 or 1982 . To be able to project to 1985 , it is necessary to estimate fertility, mortality and migration rates for each area by race, sex and age from 1980 to 1985 . Trying to estimate net migration by race, sex and age is very difficult. 


\begin{tabular}{ll} 
Comparisons of 1980 Population Projections \\
With 1980 State Level Census Data \\
\hline
\end{tabular}

Fig. 27. Results from the two population projection methodologies used in the Labor Market Projections Model are compared with 1980 Census data on population by age group at the state level. For each state and for each projection method the mean absolute percent error and percent error for the age group differing most from the census are shown.

However, this year the projections model will project to 1983. It was felt that it is not acceptable to apply a 1983 population control total to a 1980 population distribution. This distribution can change considerably over three years - particularly in areas that are growing rapidly or declining in population. Thus a third step was added to the population projections model. To project from 1980 to 1985 , national mortality rates are used. State-level fertility rates are abtained by a weighted average of the 1975-1980 state fertility rates with the national $1980-1985$ fertility rates. The procedure used to estimate the 1980-1985 net migration rates changed considerably once the 1980 Census figures on population by race were added to the model.

4) The preliminary 1980 Census Population counts were aggregated as necessary and installed in the projections model. This aggregation was accomplished with a slight modification of the geocode file and a corresponding modification in one of the Codata tools. These preliminary figures, population by race for 1980 , are used as control totals for 1980. Since these figures were felt to be of general interest, they were also installed in 
SEEDIS as a separate database. Previously they had been installed at the state, county, city, place, and med level. In this new database, they are now also available at the prime sponsor level.

5) Projecting to 1983 necessitated the addition of a third time step. The desired figures for 1983 are obtained by projecting first to 1975 , secondly to 1980 , thirdly to 1985 , and then interpolating between 1980 and 1985 to obtain 1983. Since only a total population estimate for 1985 is available, it was thought that the migration rates for 1980 1985 would have to be estimated using the plusminus technique. The migration submodel can be used only if race/sex population control totals for 1985 can be obtained or successfully estimated. However early testing of this idea shows severe problems. This is due mainly to the considerable change in racial patterns in some areas between 1970 and 1980. For example, in Prince Georges County, Maryland, the white population declined $30.4 \%$ from 1970 to 1980 , while the black population increased $171.4 \%$ over the same time period. The plus-minus technique essentially extrapolates this trend to 1985 with disastrous results. It appears that it would be better to try to estimate the 1985 population by race and sex and then use the migration model to estimate 1980-1985 migration.

With these changes, the population projections model has been greatly strengthened. As a result, the labor force and unemployment projections are similarly improved.

\section{Projects}

\section{SEEDIS/DCN}

The Socio-Economic, Environmental, Demographic Information System (SEEDIS) is an ongoing development and demonstration project addressing the need to integrate and analyze large volumes of economic, environmental, and demographic data from various sources in addressing policy issues and the use anc allocation of limited resources, both tangible and intangible. SEEDIS is installed on a network of Digital Equipment Corporation VAX-11/780 computers, and at other research sites working in collaboration with LBL in the development of analytic techniques. The network, known as the Distributed Computer Network (DCN), is shown in Figure 28. It is an experimental network established as a framework for research in networking techniques, user interfaces, and distributed information technology. The DCN project is jointly funded by DOE and the Department of Labor Employment and Training Administration. An important component of the SEEDIS/DCN project during 1981 was addition of major portions of the 1980 Census of Population and Housing. Research and development activities for SEEDIS/DCN during 1981 included investigation of data manägement in a distributed environment, development of techniques for managirnent of metadata. i... data about the databases contained in the information system, defining necessary tools and techniques for the interchange of data between heterogeneous computer systems, and enhancement of display and analysis techniques.

\section{Distributed Database Management}

During 1981 areas of jnvestigation in distributed access and control for a homogeneous network included:

- file sharing capability the ability for programs and users to access remote files;

- device sharing capability the ability to access remote peripherals;

- program sharing the ability to transmit a system to a remote computer and have it run there;

- retrieval request processing the tradeofi between remote data access for retrieval of specified data and access of complete files for processing at the host node;

- data manipulation decisions regarding remote or local processing:

- recovery and retransmission the ability to "hold" retrieval requests if remote machines or peripheral devices holding data are oft-line.

SEEDIS has implemented network retrieval for nonresident data using task-to-task communication within the DECnet facility, so that data location was transparent to end users, except for access time. In addition, whenever a SEEDIS session is initiated, it automatically initiates a process that polls the nodes and gathers information on the current location of various 


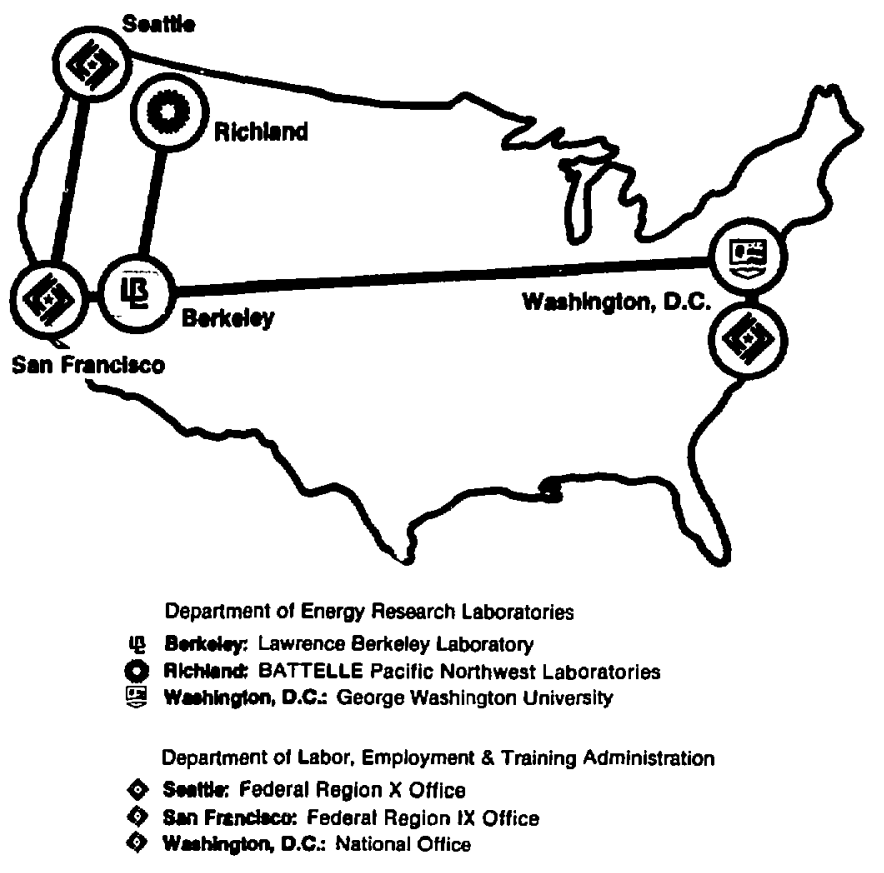

xac an1.130)

Fig. 28. The Distributed Computer Network. An experimental network of homogeneous minicomputen werves a $a$ framework for research in networking tochniques and distributed information syitems technology. LBL is linked to demonstration sites at Department of Labor. Employment and Training Administration offices and to other DOE research sites. The network provides shared scoses to the integrated databases and sophisticated date andyais and display capabilities of the SocioEconomic Environmental Demographic Information Syntem (SEEDIS).

SEEDIS disk packs and databases.

\section{Metadata Research}

When there are large numbers of databases and individual data items, it becomes dificult, if not impossible, for users to remember what data is available or what a particular data set is called. Such siluetions call for databases of information about data, called metadata, and tools to manage such metadata.

Data definition language (dd) is a nuījucucedural means to describe various aspects of data, such as physical characteristics. logical structure, statistical proper- ties, labeling information, reference documentation, security provisions, etc., at the database, data-record, data-item, and individual-item instance levels. The ddl is used initially to construct data definition files or schemas, but it can also be used to connect applications programs to databases, to generate data dictionaries and indexes, and to simplify data manipulation operations by providing information and checking facilities that would otherwise have to be built into the manipulation procedures.

Since user needs are always changing, and new needs may call for new types of metadata information. 


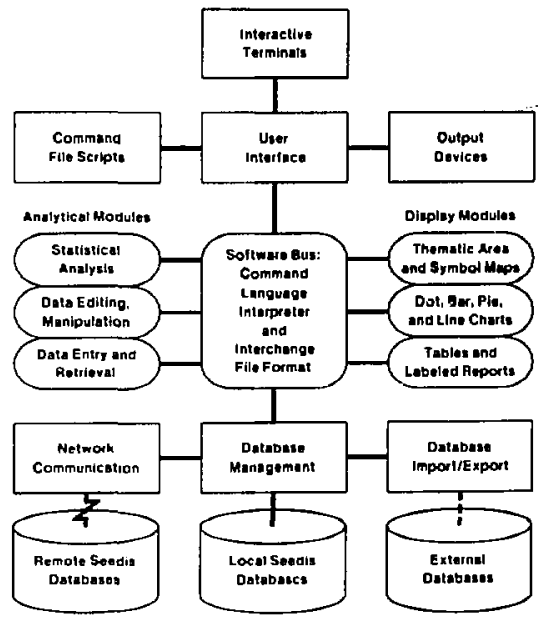

XaL Beilow

Fig. 29. A schematic of the logical structure of the SEEDIS information system.

one very important consideration is how to make the ddl sufficiently flexible so that database administrators can add new types of metadata information and revise logical structures from time to time without having to rewrite applications software or reload substantial portions of either data or metadata. The language must also be able to unambiguously define a variety of data structures and to provide a means of translating between different structures.

During the past year, LBL staff have developed specifications for an enhanced version of the SEEDIS/Codata data definition language, which will provide greater flexibility and increased ability to handie some of the more complex data structures, including multidimensional tables, hicrarchical records, and time series.

Data dictionaries or directories are the central repositories of metadata information. Dictionaries are impuriant to control and manage data, to reduce costly redundant stornge of data and metadata, and to provide a visualization of database structure and contents for varjous kinds of users.

During the past year, LBL staff have been investigating methods for automatic generation of data dictionaries from data definition files, and have been experimenting with alternative formats which can help make data easier to find and understand. For example, the current SEEDIS format for 1980 Census data dictionarics represents a substantial improvement over documentation distributed by the Census Bureau itself. and may become a future standard for similar types of documentation.

\section{Graphic Display Systems}

Development of high quality computer graphics facilities for data analysis and presentation is a major thrust of the SEEDIS project. Display systems contained in SEEDIS include CHART, a system for display of tabular data in various forms, including dot, bar, pie and line charts; and CARTE, a system for display of geographic data, including polygon (choropleth) and symbol mapping for predefined geographic entities, bivariate displays, and flow maps (Figure 30).

\section{CHART Development}

There has been considerable interest recently in new graphic forms for analysis and data display. One such form, continuous tone color shading, has been incorperated into CHART to address issues of data quality and decision making commonly encountered in energy- and labor-related data. Data such as estimates, projections, or proxy variables are often imprecise anc. uncertain, and the decision making process not well defined. A shift in analysis from the numerical values themselves to broad category ternis appropriate for each data variable, such as "high" or "low," helps diminish the misleading effects of undue precision. Another motivation for a shift in viewpoint is the opportunity for cognitive economy. Attention can be directed where judgment is most needed, and unnecessary distinctions suppressed where the issue is clear cut. This is achieved by a model for category terms based on fuzzy set theory, and a continuous tone color display to show where and how well data fit the category description.

Two application areas are being investigated. Within regional offices of the Department of Labor, these displays have been used for monitoring and evaluating prime sponsor performance, as well as for routine management information reporting. Similar displays are proposed for a geothermal energy application. An important federal policy promotes private sector exploration and development of geothermal sites on federal lands. Leasing of federal lands to conduct exploration activities is a first step that makes available identified economic resources for potential development. The displays present quantitative indicators of development potential to analysts and planners, and are designed to respond to questions such as "which geothermal sites have the best potential for successful development?" or "which sites suffer from environmental delay but otherwise have good potential?"

\section{CARTE Development}

Software for generating and maintaining cartographic base files was enhanced. A unique feature of SEEDIS is the large number of geographic levels sup- 


\begin{tabular}{|c|c|c|}
\hline Thourends of Pereons & $\begin{array}{l}\text { Source: Fodoral Tax } \\
\text { Raturna and } \\
\text { U.S. Census Bureau }\end{array}$ & $\begin{array}{l}\text { Princlpal Origins } \\
\text { and Deatinations }\end{array}$ \\
\hline
\end{tabular}

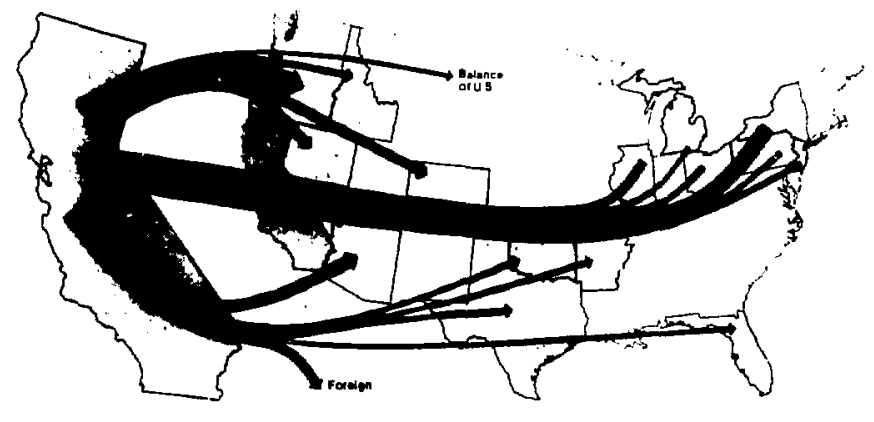

xpos a2-71s

Fig. 30. This flow map is an example of special geographic data display facilities within SEEDIS. The data is derived from the Census Bureau and Federal Income Tax returns. The flow map is generated using a few iniermediate points along the path.

poried. The great majority of these, such as SMSAs, are defined in terms of counties. Software was developed to

- Preparc a mapfile for aggregation by inserting proper geocodes;

- Perform the aggregation;

- Subtract isiand areas from surrounding polygons;

- Instrument the aggregation process to verify correctness;

- Conveniently fix any polygons in error.

Several new mapfiles were generated in this way: New England Consolidated Metropolitan Areas (NECMA) as of 1977 and 1979, Burcau of Economic Analysis areas for 1977 intersected with 1977 NECMAs.

Olier functions added to the repertoire of map munipulation tools are placing polygon labels; scaling, splitting, and joining polygors, and systematic replacement of selected polygons. These functions were used to construct a U.S. by county mapfile complete and consistent with both 1970 and 1980 county definitions. Congressional District boundaries (96th Congress) were obtained from the Census Bureau, converted to longitude/latitude, and installed in SEEDIS. Canadian census division boundary files were processed into nickel format and installed. As a by-product, the Great Lakes boundaries were extracted and can be combined with the U.S. boundary. A world map by nation from World Data Bank I was installed with nation codes according to 1980 FIPS definitions.

\section{Center for Seismic Studies}

The Center for Seismic Studies (CSS) is a Washington, DC facility supported by the Defense Advanced Research Projects Agency shown in Figure 31. The CSS has two main functions: geophysics research support and treaty validation demonstration. CSAM is involved in investigating CSS computer science proislems, thus the CSS is a prototype development of computer science research results. The three major applications of CSAM research within the CSS in 1981 were data management, local networks and graphics.

\section{Data Management}

The CSS must store and provide flexible, efficient access to two types of data: waveform and parameter 


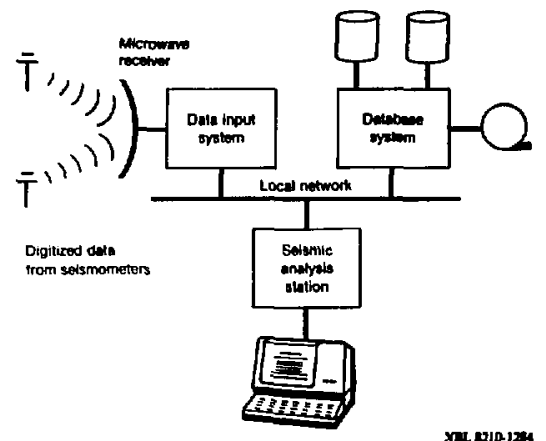

Fig. 31. The Center for Seismic Studies is a facility developed and supported by the Defense Advancod Research Projects Agency to support proposed test ban treaties and geophysics research. The CSS draw on CSAM: expertise to address long-term design and development issues.

data. Waveform data is the direct result of digitized seismometer measurements. It is transferred to the CSS via microwave broadcast, and consists of an infinite series of numbers. The amount of data coming into the CSS is about 250 Mbytes per day. This data must be archived onto tapes, indexed for casy access, and the active portions (where actual events occurred) cached separately on disk. This data is typical of time-series data and thus not amenahle to storage within most standard data management syitems (DBMS). Programs were designed during 1981 to provide the caching and indexing facilities, and to interface the indices to the parameter database.

Parameter data are data about seismic events. They consist of reports of events from various atundurd world-wide networks, reference historical data, and data generated from the analysis of on-line waveform data. Typical parameter data attributes are time, location, magnitude, compression charecteristics, etc. It was requested by the geophysical researchers that the parameter data be stored in such a way that it was accessible under any given qualification, c.g., all earthquakes within a specified longitude and latitude range, all carthquakes above a given magnitude, etc. To pro- vide such functionality, a general purpose DBMS was chosen as the correct parameter storage system. During 1981 the parameter database was designed, data loaded into it, and user interfaces to it designed and partially implemented.

\section{Local Networks}

The CSS is a modular system because it must demonstrate the feasibility of the concepts involve in the management and analysis of seismic data, and then be expandable in cose a test ban treaty is implemented. Therefore it is designed around a high-speed local network. CSAM began performance evaluation of local network technologies. Additionally, work was done with UC Berkeley UNIX implementers to design protocol implementations for the local network systems.

\section{Graphics}

The CSS is a graphics-intensive operation because the analysis of seismic data to determine location and type of seismic event still relies heavily on an analyst sitting at a display and looking at the waves generated by the event as detected at seismometers. Work was started in the design of a device and network independert graphics system for the CCS.

\section{Research Computing Facility (RCF)}

CSAM's Research Computing Facility provides the computational resources needed to support the ongoing basic research. Shown in Figure 32, these computing systems are both the object and measuring tools of the research program. The RCF serves as a testbed for deployment and evaluation of new computing capabilities. In addition to providing facilities for local researchers, the RCF is a focal point for collaborative interactions. Major changes to the facility in 1981 included upgrade of the ARPANET Interface Message Processor to the new BBN $C / 30$ configuration, as required for continued ARPANET connection after January, 1983; installation of a Britton-Lee Intelligent Database Machine (IDM S00) on loan from Brittor-Lee for experimentation, and development of local network interconnections in preparation for extensive network testing and evaluation. 


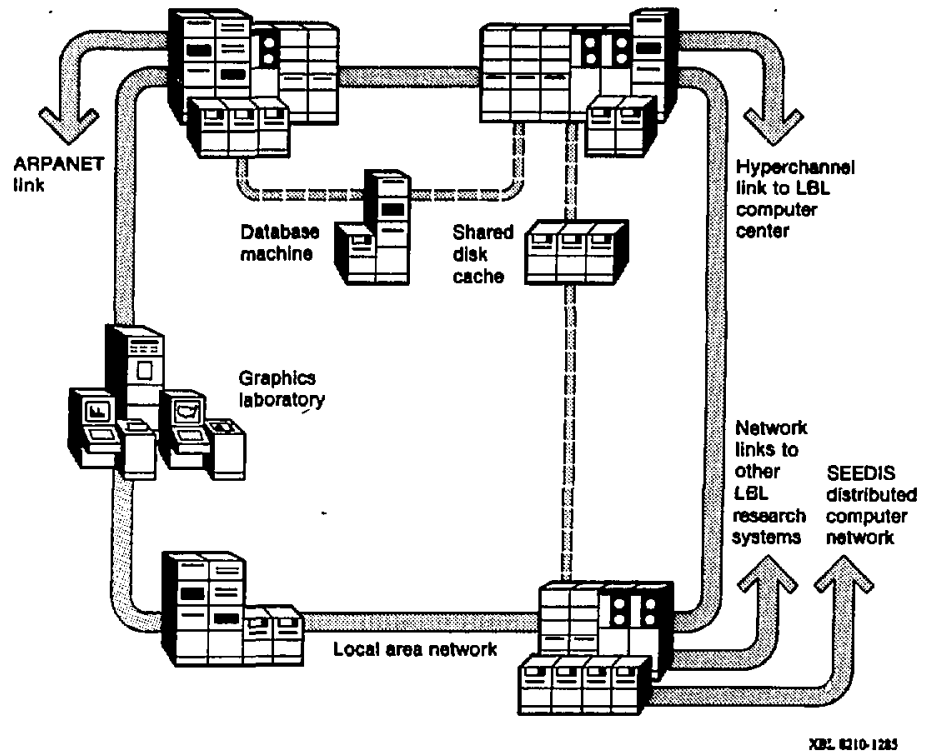

Fig. 32. CSAM's Research Computing Facility serves as a test-bed for new technologies and supports the ongoing basic research. 